\title{
COLLAPSING THREE-DIMENSIONAL CLOSED ALEXANDROV SPACES WITH A LOWER CURVATURE BOUND
}

\author{
AYATO MITSUISHI AND TAKAO YAMAGUCHI
}

Abstract. In the present paper, we determine the topologies of three-dimensional closed Alexandrov spaces which converge to lower dimensional spaces in the Gromov-Hausdorff topology.

\section{Contents}

1. Introduction

2. Preliminaries

3. Smooth approximations and flow arguments

4. The case that $\operatorname{dim} X=2$ and $\partial X=\emptyset$

5. The case that $\operatorname{dim} X=2$ and $\partial X \neq \emptyset$

6 . The case that $X$ is a circle

7. The case that $X$ is an interval

8. The case that $X$ is a single-point set

9. Appendix: $\varepsilon$-regular covering of the boundary of an Alexandrov surface

\section{INTRODUCTION}

The purpose of the present paper is to determine the topologies of collapsing three-dimensional Alexandrov spaces.

Alexandrov spaces are complete length spaces with the notion of curvature bounds. In this paper, we deal with finite dimensional Alexandrov spaces with a lower curvature bound (see Definition 2.2). Alexandrov spaces naturally appear in convergence and collapsing phenomena of Riemannian manifolds with a lower curvature bound ( $\mathrm{SY00}$, [Y 4-dim] ), and have played important roles in the study of collapsing Riemannian manifolds with a lower curvature bound.

For a positive integer $n, D>0, \kappa \in \mathbb{R}$, let us consider the following two families: $\mathcal{M}^{n}(D, \kappa)$ is the family of all isometry classes of complete $n$-dimensional Riemannian manifolds $M$ whose diameters and sectional curvatures satisfy diam $(M) \leq D$ and $\sec (M) \geq \kappa \cdot \mathcal{A}^{n}(D, \kappa)$ is the family of all isometry classes of $n$-dimensional Alexandrov spaces with diam $\leq D$ and curvature $\geq \kappa$. It follows from the definition of Alexandrov spaces that $\mathcal{M}^{n}(D, \kappa) \subset \mathcal{A}^{n}(D, \kappa)$. By Gromov's precompactness theorem, $\mathcal{A}^{n}(D, \kappa)$ has a nice property that $\bigcup_{k \leq n} \mathcal{A}^{k}(D, \kappa)$ is compact in the Gromov-Hausdorff topology, while $\bigcup_{k \leq n} \mathcal{M}^{k}(D, \kappa)$ is precompact. Therefore,

Received by the editors June 29, 2012.

2010 Mathematics Subject Classification. Primary 53C20, 53C23. 
it is quite natural to study the convergence and collapsing phenomena in $\mathcal{A}^{n}(D, \kappa)$. Thus, the following problem naturally appears:

Problem 1.1. Let $\left\{M_{i}^{n}\right\}_{i=1}^{\infty}$ be a sequence in $\mathcal{A}^{n}(D, \kappa)$ converging to an Alexandrov space $X$. Can one describe the topological structure of $M_{i}$ by using the geometry and topology of $X$ for large $i$ ?

In this paper, we consider Problem 1.1 for $n=3$ when $M_{i}$ has no boundary. We exhibit previously known results related to Problem 1.1 Let us fix the following setting: $M_{i}:=M_{i}^{n} \in \mathcal{A}^{n}(D, \kappa)$ converges to $X$ as $i \rightarrow \infty$, and fix a sufficiently large integer $i$.

If the non-collapsing case arises, i.e. $\operatorname{dim} X=n$, Perelman's stability theorem Per II (cf. Kap Stab]) shows that $M_{i}$ is homeomorphic to $X$.

In the collapsing case, we know the following results in the general dimension: If $M_{i}$ and $X$ are Riemannian manifolds, then Yamaguchi proved that there is a locally trivial fiber bundle (smooth submersion) $f_{i}: M_{i} \rightarrow X$ whose fiber is a quotient of torus by some finite group action ([Y91, Y Y conv $]$ ). Fukaya and Yamaguchi proved that if $M_{i}$ are Riemannian manifolds and $X$ is a single-point set, then $\pi_{1}\left(M_{i}\right)$ has a nilpotent subgroup of finite index [FY]. This statement also goes through even if $M_{i}$ is an Alexandrov space ( $\mathrm{Y}$ conv $]$ ).

In the lower dimensional cases, we know the following conclusive results: In dimension three, Shioya and Yamaguchi [SY00] gave a complete classification of threedimensional closed (orientable) Riemannian manifolds $M_{i}$ collapsing in $\mathcal{M}^{3}(D, \kappa)$. It is also proved that volume collapsed closed orientable Riemannian three-manifolds $M_{i}$ with no diameter bound are graph-manifolds or have small diameters and finite fundamental groups ([SY05], Per Ent]). For more recent works, see Morgan and Tian [MT, Cao and Ge CaGe, Kleiner and Lott [KL. In dimension four, Yamaguchi Y 4-dim gave a classification of four-dimensional orientable closed Riemannian manifolds $M_{i}$ collapsing in $\mathcal{M}^{4}(D, \kappa)$.

1.1. Main results. To state our results, we fix notation in this paper. $D^{n}$ is a closed $n$-disk. $D^{1}$ is written as $I$, called a (bounded closed) interval. $P^{n}$ is an $n$-dimensional real projective space. $T^{n}$ is an $n$-dimensional torus. $K^{2}$ is a Klein bottle, Mö is a Mobius band. $K^{2} \tilde{\times} I$ is an orientable (non-trivial) $I$-bundle over $K^{2} . K^{2} \hat{\times} I$ is a non-orientable non-trivial $I$-bundle over $K^{2}$. A solid Klein bottle $S^{1} \tilde{\times} D^{2}$ is obtained by $\mathbb{R} \times D^{2}$ with identification $(t, x)=(t+1, \bar{x})$. Here, we consider $D^{2}$ as the unit disk on the complex plane and $\bar{x}$ is the complex conjugate of $x$. Note that a solid Klein bottle is homeomorphic to Mö $\times I$.

A compact Alexandrov space without boundary is called closed. We classify all three-dimensional closed Alexandrov spaces collapsing to lower dimensional ones. It turns out that there is a strange phenomenon which does not occur in the manifold case. This phenomenon can be typically seen in the following example.

Example 1.2. Let $S^{1} \times \mathbb{R}^{2}$ be a flat manifold with product metric. For the isometric involution $\alpha$ defined by

$$
\alpha\left(e^{i \theta}, x\right)=\left(e^{-i \theta},-x\right),
$$

we consider the quotient space $M_{\mathrm{pt}}:=S^{1} \times \mathbb{R}^{2} /\langle\alpha\rangle$ which is an Alexandrov space with non-negative curvature. This space $M_{\mathrm{pt}}$ has the two topologically singular points, i.e. non-manifold points, $p_{+}:=[(1,0)]$ and $p_{-}:=[(-1,0)]$, which correspond to fixed points $(1,0)$ and $(-1,0)$ of $\alpha$. We consider a standard projection 
$p: M_{\mathrm{pt}} \rightarrow \mathbb{R}^{2} / x \sim-x=K\left(S_{\pi}^{1}\right)$ from $M_{\mathrm{pt}}$ to the cone $K\left(S_{\pi}^{1}\right)$ over the circle $S_{\pi}^{1}$ of length $\pi$. This is an $S^{1}$-fiber bundle over $K\left(S_{\pi}^{1}\right)$ except the vertex $o \in K\left(S_{\pi}^{1}\right)$. Remark that the fiber $p^{-1}(\partial B(o, r))$ over a metric circle at $o$ is topologically a Klein bottle. The fiber $p^{-1}(o)$ over the origin is an interval joining the topologically singular points $p_{+}$and $p_{-}$. Thus, we may regard $M_{\mathrm{pt}}$ as a circle fibration, with the singular fiber $p^{-1}(o)$, over the cone $K\left(S_{\pi}^{1}\right)$. We rescale the "circle orbits" of $M_{\mathrm{pt}}$ as $M_{\mathrm{pt}}(\varepsilon):=\left(\varepsilon S^{1}\right) \times \mathbb{R}^{2} /\langle\alpha\rangle$. Then, as $\varepsilon \rightarrow 0, M_{\mathrm{pt}}(\varepsilon)$ collapse to the cone $K\left(S_{\pi}^{1}\right)$.

We obtain the following results.

An essential singular point of an Alexandrov space is a point at which the space of directions has radius not greater than $\pi / 2$.

Theorem 1.3. Let $M_{i}^{3}$ be a sequence of three-dimensional closed Alexandrov spaces with curvature $\geq-1$ and $\operatorname{diam} M_{i} \leq D$. Suppose that $M_{i}$ converges to an Alexandrov surface $X$ without boundary. Then, for sufficiently large $i, M_{i}$ is homeomorphic to a generalized Seifert fiber space over $X$. Further, singular orbits may occur over essential singular points in $X$.

Here, a generalized Seifert fiber space is a Seifert fiber space in a generalized sense, which possibly has singular interval fibers just as in Example 1.2 For the precise definition, see Definition 2.48 .

To describe the topologies of $M_{i}^{3}$ converging to an Alexandrov surface with nonempty boundary, we define the notion of generalized solid tori and generalized solid Klein bottles. Let $K(A)$ be the cone over a topological space $A$, obtained from $A \times[0,+\infty)$ smashing $A \times\{0\}$ to a point. Let $K_{1}(A)$ be the closed cone over $A$, obtained from $A \times[0,1]$ smashing $A \times\{0\}$ to a point. We put $\partial K_{1}(A):=A \times\{1\}$.

Definition 1.4. We will construct a certain three-dimensional topological orbifold whose boundary is homeomorphic to a torus or a Klein bottle.

We first observe that the closed cone $K_{1}\left(P^{2}\right)$ over $P^{2}$ can be regarded as a "fibration" 11 over $I$ as follows. Let $\Gamma \cong \mathbb{Z}_{2}$ be the group generated by the involution $\gamma$ on $\mathbb{R}^{3}$ defined by $\gamma(v)=-v$. Then $\mathbb{R}^{3} / \Gamma=K\left(P^{2}\right)$.

We consider the following families of surfaces in $\mathbb{R}^{3}$,

$$
\begin{aligned}
& A(t):=\left\{v=(x, y, z)\left|x^{2}+y^{2}-z^{2}=t^{2},\right| z \mid \leq 1\right\} \\
& B(t):=\left\{v=(x, y, z) \mid x^{2}+y^{2}-z^{2}=-t^{2}, x^{2}+y^{2} \leq 1\right\}
\end{aligned}
$$

and set

$$
D(t):=\left\{\begin{array}{l}
A(t) / \Gamma \text { if } t>0 \\
B(t) / \Gamma \text { if } t \leq 0
\end{array}\right.
$$

Then $D(t)$ is homeomorphic to a Mobius band for $t>0$ and is homeomorphic to a disk for $t \leq 0$. Remark that $\bigcup_{t \in[-1,1]} \partial D(t)$ is homeomorphic to $S^{1} \times I$. The union $D(1) \cup \bigcup_{t \in[-1,1]} \partial D(t) \cup D(-1)$ corresponds to $P^{2} \times\{1\}=\partial K_{1}\left(P^{2}\right) \subset K_{1}\left(P^{2}\right)$. Define a projection

$$
\pi: K_{1}\left(P^{2}\right) \approx \bigcup_{t \in[-1,1]} D(t) \rightarrow[-1,1] \text { as } \pi(D(t))=t .
$$

This is a "fibration" stated as above.

\footnotetext{
${ }^{1}$ In fact, it is NOT a Serre fibration, because the fibers $D^{2}$ and Mö are not weak homotopy equivalence.
} 
For a positive integer $N \geq 1$, let us consider a circle $S^{1}=[0,2 N] /\{0\} \sim\{2 N\}$. Let $I_{j}$ be a sub-arc in $S^{1}$ corresponding to $[j-1, j] \subset[0,2 N]$ for $j=1, \ldots, 2 N$. We consider a sequence $B_{j}$ of topological spaces such that each $B_{j}$ is homeomorphic to $K_{1}\left(P^{2}\right)$. We take a sequence of projections $\pi_{j}: B_{j} \rightarrow I_{j}$ obtained as above such that there are homeomorphisms $\phi_{j}: \pi_{j}^{-1}(j) \approx \pi_{j+1}^{-1}(j)$ for all $j=1, \ldots, 2 N$. Then we obtain a topological space $Y:=\bigcup_{j=1}^{2 N} B_{j}$ glued by $\phi_{j}$ 's. Define a projection

$$
\pi: Y=\bigcup_{j=1}^{2 N} B_{j} \rightarrow S^{1} \text { by } \pi\left(\pi_{j}^{-1}(t)\right)=t
$$

for any $t \in S^{1}$. By the construction, $Y$ has $2 N$ topologically singular points. Remark that the restriction $\left.\pi\right|_{\partial Y}: \partial Y \rightarrow S^{1}$ is a usual $S^{1}$-fiber bundle. Then we obtain a topological orbifold $Y$ whose boundary $\partial Y$ is homeomorphic to a torus or a Klein bottle. If $\partial Y$ is a torus, then $Y$ is called a generalized solid torus of type $N$. If $\partial Y$ is a Klein bottle, then $Y$ is called a generalized solid Klein bottle of type $N$. We regard a solid torus $S^{1} \times D^{2}$ and the product $S^{1} \times$ Mö as generalized solid tori of type 0 . We also regard a solid Klein bottle $S^{1} \tilde{\times} D^{2}$ and non-trivial Mö-bundle $S^{1} \tilde{\times}$ Mö over $S^{1}$ as generalized solid Klein bottles of type 0 . Note that $S^{1} \tilde{\times}$ Mö is homeomorphic to a non-orientable $I$-bundle $K^{2} \hat{x} I$ over $K^{2}$.

For a two-dimensional Alexandrov space $X$, a boundary point $x \in \partial X$ is called a corner point if diam $\Sigma_{x} \leq \pi$, in other words, if it is an essential singular point.

Theorem 1.5. Let $\left\{M_{i}\right\}_{i=1}^{\infty}$ be a sequence of three-dimensional closed Alexandrov spaces with curvature $\geq-1$ and $\operatorname{diam} M_{i} \leq D$. Suppose that $M_{i}$ converges to an Alexandrov surface $X$ with non-empty boundary. Then, for large $i$, there exist a generalized Seifert fiber space $\operatorname{Seif}_{i}(X)$ over $X$ and generalized solid tori or generalized solid Klein bottles $\pi_{i, k}: Y_{i, k} \rightarrow(\partial X)_{k}$ over each component $(\partial X)_{k}$ of $\partial X$ such that $M_{i}$ is homeomorphic to a union of $\operatorname{Seif}_{i}(X)$ and $Y_{i, k}$ 's glued along their boundaries, where the fibers of $\operatorname{Seif}_{i}(X)$ over a boundary points $x \in(\partial X)_{k}$ are identified with $\partial \pi_{i, k}^{-1}(x) \approx S^{1}$.

It should be remarked that in Theorem 1.5, the fiber of $\pi_{i, k}: Y_{i, k} \rightarrow(\partial X)_{k}$ may change at a corner point of $(\partial X)_{k}$ and that the type of $Y_{i, k}$ is less than or equal to half of the number of corner points in $(\partial X)_{k}$.

Corollary 1.6. Under the same assumption and notation of Theorem 1.5, for large $i$, there exists a continuous surjection $f_{i}: M_{i} \rightarrow X$ which is a $\theta(i)$-approximation satisfying the following:

(1) $f_{i}: f_{i}^{-1}(\operatorname{int} X) \rightarrow \operatorname{int} X$ is a generalized Siefert fibration.

(2) For $x \in \partial X, f_{i}^{-1}(x)$ is homeomorphic to a one-point set or a circle. The fiber of $f_{i}$ may change over a corner point in $\partial X$.

(3) For any collar neighborhood $\varphi:(\partial X)_{k} \times[0,1] \rightarrow X$ of a component $(\partial X)_{k}$ of $\partial X$, which contains no interior essential singular points, $f_{i}^{-1}$ (image $\varphi$ ) is a generalized solid torus or a generalized solid Klein bottle.

Using the same notation as in Corollary 1.6, we remark that, for $x \in(\partial X)_{k}$,

$$
\begin{aligned}
& f_{i}^{-1}(\varphi(\{x\} \times[0,1])) \approx D^{2} \text { if } f_{i}^{-1}(x) \approx\{\mathrm{pt}\}, \\
& f_{i}^{-1}(\varphi(\{x\} \times[0,1])) \approx \text { Mö if } f_{i}^{-1}(x) \approx S^{1} .
\end{aligned}
$$

The structure of $M_{i}$ collapsing to one-dimensional space is determined as follows. 
Theorem 1.7. Let $M_{i}^{3}$ be a sequence of three-dimensional closed Alexandrov spaces with curvature $\geq-1$ and $\operatorname{diam} M_{i} \leq D$. Suppose that $M_{i}^{3}$ converges to a circle. Then, for large $i, M_{i}$ is homeomorphic to a total space of an $F_{i}$-fiber bundle over $S^{1}$, where the fiber $F_{i}$ is homeomorphic to one of $S^{2}, P^{2}, T^{2}$ and $K^{2}$.

To describe the structures of $M_{i}$ converging to an interval $I$, we prepare certain topological orbifolds. First, we provide

$$
B(\mathrm{pt}):=S^{1} \times D^{2} /\langle\alpha\rangle .
$$

Here, the involution $\alpha$ is the restriction of the one provided in Example 1.2 Remark that $\partial B(\mathrm{pt}) \approx S^{2}$. We also need to consider three-dimensional open Alexandrov spaces $L_{2}$ and $L_{4}$ with two-dimensional souls $S_{2}$ and $S_{4}$ respectively, where $S_{2}$ (resp. $S_{4}$ ) is homeomorpshic to $S^{2}$ or $P^{2}$ (resp. to $S^{2}$ ). For their definition, see Example 2.63. The space $L_{i}(i=2,4)$ has $i$ topologically singular points, which are contained in $S_{i}$. We denote by $B\left(S_{i}\right)$ a metric ball around $S_{i}$ in $L_{i}$. Here we point out that $\partial B\left(S_{2}\right) \approx S^{2}$ (resp. $\left.\approx K^{2}\right)$ if $S_{2} \approx S^{2}$ (resp. if $\left.S_{2} \approx P^{2}\right)$, and $\partial B\left(S_{4}\right) \approx T^{2}$.

Theorem 1.8. Let $M_{i}^{3}$ be a sequence of three-dimensional closed Alexandrov spaces with curvature $\geq-1$ and diam $M_{i} \leq D$. Suppose that $M_{i}^{3}$ converges to an interval. Then, for large $i, M_{i}$ is the union of $B_{i} \cup B_{i}^{\prime}$ glued along their boundaries. $\partial B_{i}$ is homeomorphic to one of $S^{2}, P^{2}, T^{2}$ and $K^{2}$. The topologies of $B_{i}$ (and $B_{i}^{\prime}$ ) are determined as follows:

(1) If $\partial B_{i} \approx S^{2}$, then $B_{i}$ is homeomorphic to one of $D^{3}, P^{3}-\operatorname{int} D^{3}, B\left(S_{2}\right)$ with $S_{2} \approx S^{2}$.

(2) If $\partial B_{i} \approx P^{2}$, then $B_{i}$ is homeomorphic to $K_{1}\left(P^{2}\right)$.

(3) If $\partial B_{i} \approx T^{2}$, then $B_{i}$ is homeomorphic to one of $S^{1} \times D^{2}, S^{1} \times \mathrm{Mö}, K^{2} \tilde{\times} I$, and $B\left(S_{4}\right)$.

(4) If $\partial B_{i} \approx K^{2}$, then $B_{i}$ is homeomorphic to one of $S^{1} \tilde{\times} D^{2}, K^{2} \hat{\times} I, B(\mathrm{pt})$, and $B\left(S_{2}\right)$ with $S_{2} \approx P^{2}$.

Corollary 1.9. Let $M_{i}$ be a sequence of three-dimensional closed Alexandrov spaces with curvature $\geq-1$ and diameter $\leq D$. Suppose $M_{i}$ converges to a point. Then, for large $i, M_{i}$ is homeomorphic to one of

- generalized Seifert fiber spaces in the conclusion of Theorem 1.3 with a base Alexandrov surface having non-negative curvature,

- spaces in the conclusion of Theorem 1.5 with a base Alexandrov surface having non-negative curvature,

- spaces in the conclusion of Theorems 1.7 and 1.8, and

- closed Alexandrov spaces with non-negative curvature having finite fundamental groups.

We remark that all spaces appearing in the conclusions of Theorems 1.3, 1.5, 1.7 and 1.8 and Corollary 1.9 actually have sequences of metrics as Alexandrov spaces collapsing to such respective limit spaces described there.

By Corollary [1.9, to achieve a complete classification of the topologies of collapsing three-dimensional closed Alexandrov spaces, we provide a version of the "Poincaré conjecture" for three-dimensional closed Alexandrov spaces with nonnegative curvature.

For Alexandrov spaces $A$ and $A^{\prime}$ having boundaries isometric to each other, $A \cup_{\partial} A^{\prime}$ denotes the gluing of $A \cup A^{\prime}$ via an isometry $\phi: \partial A \rightarrow \partial A^{\prime}$. Note that $A \cup_{\partial} A^{\prime}$ is an Alexandrov space (see Pet Appl]). 
Conjecture 1.10. A simply connected three-dimensional closed Alexandrov space with non-negative curvature is homeomorphic to an isometric gluing $A \cup_{\partial} A^{\prime}$ for $A$ and $A^{\prime}$ chosen in the following list (1.3) of non-negatively curved Alexandrov spaces:

$$
D^{3}, K_{1}\left(P^{2}\right), B(\mathrm{pt}), B\left(S_{2}\right), B\left(S_{4}\right) .
$$

We also remark that any connected sum of those spaces admits a metric of Alexandrov space having a lower curvature bound by some constant.

Conjecture 1.11. A simply connected three-dimensional closed Alexandrov space with curvature $\geq 1$ is homeomorphic to a three-sphere $S^{3}$ or a suspension $\Sigma\left(P^{2}\right)$ over $P^{2}$.

The organization of this paper and basic ideas of the proofs of our results are as follows:

In Section 2, we review some basic notation and results on Alexandrov spaces. We provide a three-dimensional topological orbifold having a circle fiber structure with singular arc fibers, and call it a generalized Seifert fiber space. At the end of this section, we prove fundamental properties on the topologically singular point set.

In Section 3, for any $n \in \mathbb{N}$, we consider $n$-dimensional closed Alexandrov spaces $M_{i}^{n}$ collapsing to a space $X^{n-1}$ of co-dimension one. Assume that all points in $X$ are almost regular, except finite points $x_{1}, \ldots, x_{m}$. For any fixed $p \in\left\{x_{\alpha}\right\}$, we take a sequence $p_{i} \in M_{i}$ converging to $p$. By Yamaguchi's Fibration Theorem 2.25. for large $i$, there is a fiber bundle $\pi_{i}: A_{i} \rightarrow A$, where $A$ is a small metric annulus $A=A(p ; r, R)$ around $p$ and $A_{i}$ is some corresponding domain. Here, $r$ and $R$ are small positive numbers so that $r \ll R$.

Although $A_{i}$ is not a metric annulus in general, it is expected that $A_{i}$ is homeomorphic to a standard annulus $A\left(p_{i} ; r, R\right)$. Moreover, we may expect that there exist an isotopy $\phi: M_{i} \times[0,1] \rightarrow M_{i}$ such that, putting $\phi_{t}:=\phi(\cdot, t)$,

$$
\left\{\begin{array}{l}
\phi_{0}=i d_{M_{i}}, \\
\phi_{1}\left(B\left(p_{i}, \frac{r+R}{2}\right) \cup A_{i}\right)=B\left(p_{i}, R\right), \text { and } \\
\phi_{1}(x)=x \text { if } x \notin B\left(p_{i}, R+\delta\right)
\end{array}\right.
$$

for any fixed $\delta>0$.

If we consider the case that all $M_{i}$ are Riemannian manifolds, then we can obtain a smooth flow $\Phi_{t}$ of a gradient-like vector field $V$ of the distance function dist $p_{i}$ from $p_{i}$. Then, by using integral curves of $V$, we can obtain such an isotopy $\phi$ from $i d_{M_{i}}$ satisfying the property (1.4).

We will prove that such an argument of flow goes through on Alexandrov spaces $M_{i}$ as well. To do this, we first prove a main result, Flow Theorem 3.2 , in this section. Theorem 3.2 implies the existence of an integral flow $\Phi_{t}$ of a gradient-like vector field of a distance function $\operatorname{dist}_{p_{i}}$ on $A\left(p_{i} ; r, R\right)$ in a suitable sense. This flow leads to an isotopy $\phi$ satisfying the property (1.4). Theorem 3.2 is important throughout the paper.

In Sections 4-8, we prove Theorems 1.3, 1.5, 1.7 and 1.8 and Corollaries 1.6 and 1.9. To explain the arguments used in those proofs, let us fix a sequence $M_{i}=M_{i}^{3}$ of three-dimensional closed Alexandrov spaces in $\mathcal{A}^{3}(-1, D)$ converging to $X$ of dimension $\leq 2$. 
In Section 4, we consider the case that $\operatorname{dim} X=2$ and $\partial X=\emptyset$. Let $p_{1}, \ldots, p_{m}$ be all $\delta$-singular points in $X$ for a fixed small $\delta>0$. Let us take a converging sequence $p_{i, \alpha} \rightarrow p_{\alpha}(i \rightarrow \infty)$ for each $\alpha=1, \ldots, m$. Let us fix any $\alpha$ and set $p:=p_{\alpha}$, $p_{i}:=p_{i, \alpha}$. We take $r=r_{p}>0$ such that all points in $B(p, 2 r)-\{p\}$ are $(2, \varepsilon)$ strained. Then, all points in an annulus $A\left(p_{i} ; \varepsilon_{i}, 2 r-\varepsilon_{i}\right)$ are $(3, \theta(i, \varepsilon))$-strained. Here, $\varepsilon_{i}$ is a sequence of positive numbers converging to zero. Then, by Fibration Theorem 2.25, we have an $S^{1}$-fiber bundle $\pi_{i}: A_{i} \rightarrow A(p ; r, 2 r)$. On the other hand, by the rescaling argument 2.27, we obtain the conclusion that $B_{i}:=B\left(p_{i}, r\right)$ is homeomorphic to a solid torus or $B(\mathrm{pt})$. Here, we can exclude the possibility that $B_{i}$ is topologically a solid Klein bottle. Theorem 3.2 implies that there exists an isotopy carrying the fiber $\pi_{i}^{-1}(\partial B(p, r))$ to $\partial B_{i}$. If $B_{i} \approx S^{1} \times D^{2}$ then we can prove an argument similar to SY00 that $B_{i}$ has the structure of a Seifert fibered torus in the usual sense, extending $\pi$. If $B_{i} \approx B(\mathrm{pt})$, then by some new observation on the topological structure of $B(\mathrm{pt})$, we can prove that $B_{i}$ has the standard "circle fibration" structure provided in Example 1.2, compatible with $\pi$. In this way, we obtain the structure of a generalized Seifert fiber space on $M_{i}$.

In Section 5. we consider the case that $\operatorname{dim} X=2$ and $\partial X \neq \emptyset$. Take a decomposition of $\partial X$ to connected components $\bigcup_{\beta}(\partial X)_{\beta}$. Put $X_{0}:=X-U(\partial X, r)$ for some small $r>0$. By Theorem 1.3, we have a generalized Seifert fibration $\pi_{i}: M_{i, 0} \rightarrow X_{0}$ for some closed domain $M_{i, 0} \subset M_{i}$. For any fixed $\beta$, we take points $p_{\alpha}$ in $(\partial X)_{\beta}$ so fine that $\left\{p_{\alpha}\right\}$ contains all $\varepsilon$-singular points in $(\partial X)_{\beta}$. Let $p_{i, \alpha} \in M_{i}$ be a sequence converging to $p_{\alpha}$. Deform a metric ball $B\left(p_{i, \alpha}, r\right)$ to a neighborhood $B_{i, \alpha}$ of $p_{i, \alpha}$ by an isotopy obtained in Theorem 3.2. Because of the existence of $\partial X$, we need a bit more complicated construction of flows of gradient-like vector fields of distance functions.

In Section 6, we consider the case that $X$ is isometric to a circle $S^{1}(\ell)$ of length $\ell$. If $M_{i}$ has no $\varepsilon$-singular points, by Fibration Theorem 2.25, we obviously obtain the conclusion of Theorem 1.7. But, in general, $M_{i}$ has $\varepsilon$-singular points. Therefore, we use Perelman's Morse theory to construct a fibration over $S^{1}$.

In Section [7, we consider the case that $X$ is isometric to an interval $[0, \ell]$ of some length $\ell$. We use rescaling arguments around the end points of interval $X$ and an argument similar to Theorem 1.7 to prove Theorem 1.8.

In Section 8, we consider the case of $\operatorname{dim} X=0$ and prove Corollary 1.9 ,

For three-dimensional Alexandrov spaces with non-empty boundary collapsing to lower dimensional spaces, considering their doubles, one could make use of the results in the present paper to obtain the structure of collapsing in that case. This will appear in a forthcoming paper.

\section{Preliminaries}

2.1. Definitions, conventions and notation. In the present paper, we use the following notation.

- $\theta(\delta)$ is a function depending on $\delta=\left(\delta_{1}, \ldots, \delta_{k}\right)$ such that $\lim _{\delta \rightarrow 0} \theta(\delta)$ $=0 . \quad \theta(i, \delta)$ is a function depending on $\delta \in \mathbb{R}^{k}$ and $i \in \mathbb{N}$ such that $\lim _{i \rightarrow \infty, \delta \rightarrow 0} \theta(i, \delta)=0$. When we write $A<\theta(\delta)$ for a non-negative number $A$, we always assume that $\theta(\delta)$ is taken to be non-negative.

- $X \approx Y$ means that $X$ is homeomorphic to $Y$. For metric spaces $X$ and $Y$, $X \equiv Y$ means that $X$ is isometric to $Y$. 
- For metric spaces $X$ and $Y$, the direct product $X \times Y$ has the product metric if nothing is stated.

- For continuous mappings $f_{1}: X_{1} \rightarrow Y, f_{2}: X_{2} \rightarrow Y$ and $g: X_{1} \rightarrow X_{2}$, we say that $g$ represents $f_{1}$ and $f_{2}$ if $f_{1}=f_{2} \circ g$ holds.

- Denote by $d(x, y),|x, y|$, and $|x y|$ the distance between $x$ and $y$ in a metric space $X$. Sometimes we mark $X$ as lower index $|x, y|_{X}$.

- For a subset $S$ of a topological space, $\bar{S}$ is the closure of $S$ in the whole space.

- For a metric space $X=(X, d)$ and $r>0$, denote the rescaling metric space $r X=(X, r d)$.

- For a subset $Y$ of a metric space, denote by dist $_{Y}$ the distance function from $Y$. When $Y=\{x\}$ we denote $\operatorname{dist}_{x}:=\operatorname{dist}_{\{x\}}$. For a subset $Y$ of a metric space $X$ and a subset $I$ of $\mathbb{R}_{+}$, define a subset $B(Y ; I):=B_{X}(Y ; I):=$ $\operatorname{dist}_{Y}^{-1}(I) \subset X$. For special cases, we denote and call those sets in the following way: $B(Y, r):=B(Y ;[0, r])$ the closed ball, $U(Y, r):=B(Y ;[0, r))$ the open ball, $A\left(Y ; r^{\prime}, r\right):=B\left(Y ;\left[r^{\prime}, r\right]\right)$ the annulus, and $\partial B(Y, r):=$ $B(Y ;\{r\})$ the metric sphere. For $Y=\{x\}$, we set $B(x, r):=B(\{x\}, r)$, $U(x, r):=U(\{x\}, r)$ and $A\left(x ; r^{\prime}, r\right):=A\left(\{x\} ; r^{\prime}, r\right)$.

- For a topological space $X$, the cone $K(X)$ over $X$ is obtained from $X \times$ $[0, \infty)$ by smashing $X \times\{0\}$ to a point. An equivalent class $[(x, a)] \in K(X)$ of $(x, a) \in X \times[0,+\infty)$ is denoted by ax or often simply written by $(x, a)$. A special point $(x, 0)=0 x \in K(X)$ is denoted by $o$ or $o_{X}$, called the origin of $K(X)$. A point $v \in K(X)$ is often called a vector. $K_{1}(X)$ denotes the (unit) closed cone over $X$, i.e.

$$
K_{1}(X):=\{a x \in K(X) \mid x \in X, 0 \leq a \leq 1\} .
$$

$K_{1}(X)$ is homeomorphic to the join between $X$ and a single-point.

- For a metric space $X, K(X)$ often denotes the Euclidean metric cone, which is equipped with the following metric: for two points $\left(x_{1}, r_{1}\right),\left(x_{2}, r_{2}\right) \in$ $X \times[0, \infty)$ the distance between them is defined by

$$
d\left(\left(x_{1}, r_{1}\right),\left(x_{2}, r_{2}\right)\right)^{2}:=r_{1}^{2}+r_{2}^{2}-2 r_{1} r_{2} \cos \min \left\{d\left(x_{1}, x_{2}\right), \pi\right\} .
$$

And for $v \in K(X)$, we put $|v|:=d(x, o)$ and call it the norm of $v$. Define an inner product $\langle v, w\rangle$ of $v, w \in K(X)$ by $\langle v, w\rangle:=|v||w| \cos \angle v o w$.

- When we write $M^{n}$ marked upper index $n$, this means that $M$ is an $n$ dimensional Alexandrov space.

For a curve $\gamma:[0,1] \rightarrow X$ in a metric space $X$, the length $L(\gamma)$ of $\gamma$ is defined by

$$
L(\gamma):=\sup _{0=t_{0}<t_{1}<\cdots<t_{m}=1} \sum_{i=1}^{m} d\left(\gamma\left(t_{i-1}\right), \gamma\left(t_{i}\right)\right) \in[0,+\infty] .
$$

A metric space $X$ is called a length space if for any $x, y \in X$ and $\varepsilon>0$, there exists a curve $\gamma:[0,1] \rightarrow X$ such that $\gamma(0)=x, \gamma(1)=y$ and $0 \leq L(\gamma)-d(x, y) \leq \varepsilon$. A curve is called a geodesic if it is an isometric embedding from some interval. Sometimes a geodesic $\gamma$ defined on a bounded closed interval $[0, \ell]$ is called a geodesic segment. A geodesic defined on $\mathbb{R}$ is called a line; a geodesic defined on $[0,+\infty)$ is called a ray. For a geodesic $\gamma: I \rightarrow X$ in a metric space $X$, we often regard $\gamma$ itself as the subset $\gamma(I) \subset X$. 
2.2. Alexandrov spaces. From now on, throughout this paper, we always assume that a metric space is proper, namely, any closed bounded subset is compact. A proper length space is a geodesic space, namely, any two points are jointed by a geodesic.

For three points $x_{0}, x_{1}, x_{2}$ in a metric space, the size of $\left(x_{0}, x_{1}, x_{2}\right)$ is size $\left(x_{0}, x_{1}, x_{2}\right):=\left|x_{0} x_{1}\right|+\left|x_{1} x_{2}\right|+\left|x_{2} x_{0}\right|$. The size of four points $\left(x_{0} ; x_{1}, x_{2}, x_{3}\right)$ (centered at $\left.x_{0}\right)$ is defined by the maximum of size $\left(x_{0}, x_{i}, x_{j}\right)$ for $1 \leq i \neq j \leq 3$, denoted by size $\left(x_{0} ; x_{1}, x_{2}, x_{3}\right)$.

Definition 2.1. For three points $x_{0}, x_{1}, x_{2}$ in a metric space $X$ with size $\left(x_{0}, x_{1}, x_{2}\right)$ $<2 \pi / \sqrt{\kappa}$, the $\kappa$-comparison angle of $\left(x_{0} ; x_{1}, x_{2}\right)$, written by $\tilde{L}_{\kappa} x_{1} x_{0} x_{2}$ or $\tilde{L}_{\kappa}\left(x_{0} ; x_{1}, x_{2}\right)$, is defined as follows: Take three points $\tilde{x}_{i}(i=0,1,2)$ in $\kappa$-plane $\mathbb{M}_{\kappa}^{2}$, which is a simply connected complete surface with constant curvature $=\kappa$, such that $d\left(x_{i}, x_{j}\right)=d\left(\tilde{x}_{i}, \tilde{x}_{j}\right)$ for $0 \leq i, j \leq 2$ and put $\tilde{L}_{\kappa} x_{1} x_{0} x_{2}:=\angle \tilde{x}_{1} \tilde{x}_{0} \tilde{x}_{2}$. Sometimes we write $\tilde{L}$ omitting $\kappa$ in the notation $\tilde{L}_{\kappa}$.

Definition 2.2. For $\kappa \in \mathbb{R}$, a complete metric space $X$ is called an Alexandrov space with curvature $\geq \kappa$ if $X$ is a length space and, for every four points $x_{0}, x_{1}, x_{2}, x_{3} \in X$ (with size $\left(x_{0} ; x_{1}, x_{2}, x_{3}\right)<2 \pi / \sqrt{\kappa}$ if $\kappa>0$ ), we have the inequality

$$
\tilde{L}_{\kappa} x_{1} x_{0} x_{2}+\tilde{L}_{\kappa} x_{2} x_{0} x_{3}+\tilde{L}_{\kappa} x_{3} x_{0} x_{1} \leq 2 \pi .
$$

The dimension of an Alexandrov space means its Hausdorff dimension. The Hausdorff dimension and the topological dimension are equal to each other ( $\mathrm{BGP}$, [PP QG], [Pl]). Throughout this paper, we always assume that an Alexandrov space is finite dimensional.

Remark 2.3. If $X$ is an Alexandrov space with curvature $\geq \kappa$, then the rescaling space $r X$ is an Alexandrov space with curvature $\geq \kappa / r^{2}$.

For two geodesics $\alpha, \beta:[0, \varepsilon] \rightarrow X$ emanating at $\alpha(0)=\beta(0)=p \in X$ in an Alexandrov space $X$, the angle $\angle(\alpha, \beta)$ at $p$ is defined by

$$
\angle(\alpha, \beta):=\angle_{p}(\alpha, \beta):=\lim _{s, t \rightarrow 0} \tilde{\angle}(p ; \alpha(t), \beta(s)) .
$$

The set of all non-trivial geodesics emanating at $p$ in an Alexandrov space $X$ is denoted by $\Sigma_{p}^{\prime} X$. The angle $\angle_{p}$ at $p$ satisfies the triangle inequality on this set. Its metric completion is denoted by $\Sigma_{p}=\Sigma_{p} X$, called the space of directions at $p$. For a geodesic $\gamma:[0, \ell] \rightarrow X$ starting from $x=\gamma(0)$ to $y=\gamma(\ell)$, we denote $\gamma^{+}(0)=\gamma^{\prime}(0)=\gamma_{x}^{\prime}=\gamma_{x}^{+}=\uparrow_{x}^{y}$ the direction of $\gamma$ at $x$. By $x y$, we denote some segment $x y=\gamma:[0,|x y|] \rightarrow X$ joining from $\gamma(0)=x$ to $\gamma(|x y|)=y$. For a subset $A \subset X$, the closure of a set of all directions from $x$ to $A$ is denoted by $A_{x}^{\prime}$, i.e.

$$
A_{x}^{\prime}:=\left\{\xi \in \Sigma_{x} \mid \exists a_{i} \in A \text { such that } \lim _{i \rightarrow \infty}\left|x a_{i}\right|=|x, A| \text { and } \lim _{i \rightarrow \infty} \uparrow_{x}^{a_{i}}=\xi\right\} .
$$

When $x \in A$, we put $\Sigma_{x}(A):=A_{x}^{\prime}$. For $x, y \in X$, we denote as $y_{x}^{\prime}:=\{y\}_{x}^{\prime}$. Or sometimes we denote by $y_{x}^{\prime}$ an element that belongs with $y_{x}^{\prime}$. For $x \in X$ and $y, z \in X-\{x\}$, we denote by $\angle y x z$ the angle $\angle(x y, x z)=\angle\left(\uparrow_{x}^{y}, \uparrow_{x}^{z}\right)$ between some fixed segments $x y, x z$. 
Definition 2.4. A $(k, \delta)$-strainer at $x \in M$ is a collection of points $\left\{p_{\alpha}^{ \pm}\right\}_{\alpha=1}^{k}=$ $\left\{p_{\alpha}^{+}, p_{\alpha}^{-} \mid \alpha=1, \ldots, k\right\}$ satisfying the following:

$$
\begin{aligned}
& \tilde{\angle} p_{\alpha}^{+} x p_{\beta}^{+}>\pi / 2-\delta \\
& \tilde{\angle} p_{\alpha}^{+} x p_{\beta}^{-}>\pi / 2-\delta \\
& \tilde{\angle} p_{\alpha}^{-} x p_{\beta}^{-}>\pi / 2-\delta \\
& \tilde{\angle} p_{\alpha}^{+} x p_{\alpha}^{-}>\pi-\delta
\end{aligned}
$$

for all $1 \leq \alpha \neq \beta \leq k$.

The length of a strainer $\left\{p_{\alpha}^{ \pm}\right\}$at $x$ is $\min _{1 \leq \alpha \leq k}\left\{\left|p_{\alpha}^{+}, x\right|,\left|p_{\alpha}^{-}, x\right|\right\}$. The $(k, \delta)$ strained radius of $x$, denoted by $(k, \delta)$-str.rad $x$, is the supremum of lengths of $(k, \delta)$-strainers at $x . \mathrm{A}(k, \delta)$-strained radius $(k, \delta)$-str.rad $A$ of a subset $A \subset M$ is defined by

$$
(k, \delta) \text {-str.rad } A:=\inf _{x \in A}(k, \delta) \text {-str.rad } x .
$$

If there is a $(k, \delta)$-strainer at $x$, then $x$ is called $(k, \delta)$-strained. Denote by $R_{k, \delta}(M)$ the set of all $(k, \delta)$-strained points in $M . R_{k, \delta}(M)$ is an open subset. Put $S_{k, \delta}(M):=$ $M-R_{k, \delta}(M)$. Any point in $S_{k, \delta}(M)$ is called a $(k, \delta)$-singular point. When we consider an $n$-dimensional Alexandrov space $M^{n}$ and $\delta$ is sufficiently small with respect to $1 / n$, we simply say $\delta$-strained, $\delta$-singular, etc., instead of $(n, \delta)$-strained, $(n, \delta)$ singular, etc., and we omit writing $R_{\delta}(M), S_{\delta}(M)$ instead of $R_{n, \delta}(M), S_{n, \delta}(M)$. For an $n$-dimensional Alexandrov space $M^{n}$, put $R\left(M^{n}\right):=\bigcap_{\delta>0} R_{\delta}\left(M^{n}\right)$ and $S\left(M^{n}\right):=\bigcup_{\delta>0} S_{\delta}\left(M^{n}\right)=M^{n}-R\left(M^{n}\right)$.

Theorem 2.5 ([BGP], $\mathrm{OS}])$. For any $n$-dimensional Alexandrov space $M^{n}$, we have $\operatorname{dim}_{H} S(M) \leq n-1$ and $\operatorname{dim}_{H} S(M)-\partial M \leq n-2$.

Here, the boundary $\partial M$ of an Alexandrov space $M$ is defined inductively in the following manner.

Definition 2.6. A one-dimensional Alexandrov space $M^{1}$ is a manifold, and the boundary of $M^{1}$ is the boundary of $M^{1}$ as a manifold. Now let $M^{n}$ be an $n$ dimensional Alexandrov space with $n>1$. A point $p$ in $M^{n}$ is called a boundary point if $\Sigma_{p}$ has a boundary point. The set of all boundary points is denoted by $\partial M^{n}$, called the boundary of $M^{n}$. Its complement is denoted by int $M^{n}=M^{n}-\partial M^{n}$, called the interior of $M^{n}$. A point in int $M^{n}$ is called an interior point of $M^{n}$. $\partial M^{n}$ is a closed subset in $M^{n}$ ([BGP, Per II $]$ ).

A compact Alexandrov space without boundary is called a closed Alexandrov space, and a non-compact Alexandrov space without boundary is called an open Alexandrov space.

Definition 2.7. For an $n$-dimensional Alexandrov space $M^{n}$, we say that $p \in M$ is a topologically regular point (or a manifold-point) if there is a neighborhood of $p$ which is homeomorphic to $\mathbb{R}^{n}$ or $\mathbb{R}^{n-1} \times[0, \infty)$. $p$ is called a topologically singular point if $p$ is not a topologically regular point. We denote by $S_{\text {top }}(M)$ the set of all topologically singular points.

Definition 2.8. For an Alexandrov space $M$, a point $p \in M$ is called an essential singular point if $\operatorname{rad} \Sigma_{p} \leq \pi / 2$. A set of whole essential singular points in $M$ is denoted by $\operatorname{Ess}(M)$. We define the set of interior (resp. boundary) essential 
singular points $\operatorname{Ess}(\operatorname{int} M)$ (resp. $\operatorname{Ess}(\partial M))$ as follows:

$$
\begin{aligned}
\operatorname{Ess}(\operatorname{int} M) & :=\operatorname{Ess}(M) \cap \operatorname{int} M, \\
\operatorname{Ess}(\partial M) & :=\operatorname{Ess}(M) \cap \partial M .
\end{aligned}
$$

Remark that if $\operatorname{dim} M=1$, then $\operatorname{Ess}(\operatorname{int} M)=\emptyset$ and $\operatorname{Ess}(\partial M)=\partial M$.

Remark 2.9. By Theorem 2.36 and Stability Theorem 2.34, we can check the following:

$$
S_{\text {top }}(M) \subset \operatorname{Ess}(M) \subset S(M) .
$$

For small $\delta \ll 1 / n$, any $(n, \delta)$-regular point in an $n$-dimensional Alexandrov space $M^{n}$ is an interior point.

Theorem 2.10 ([BGP, Corollary 12.8]). An $(n-1, \delta)$-regular interior point in an $n$-dimensional Alexandrov space is an $\left(n, \delta^{\prime}\right)$-regular point. Here, $\delta^{\prime} \rightarrow 0$ as $\delta \rightarrow 0$.

The boundary of an Alexandrov space is determined by its topology:

Theorem 2.11 ([BGP, Theorem 13.3(a)], [Per II]). Let $M_{1}, M_{2}$ be $n$-dimensional Alexandrov spaces with homeomorphism $\phi: M_{1} \rightarrow M_{2}$. Then $\phi\left(\partial M_{1}\right)=\partial M_{2}$.

2.3. The Gromov-Hausdorff convergence. For metric spaces $X$ and $Y$, and $\varepsilon>0$, an $\varepsilon$-approximation $f$ from $X$ to $Y$ is a map $f: X \rightarrow Y$ such that

(1) $\left|d\left(x, x^{\prime}\right)-d\left(f(x), f\left(x^{\prime}\right)\right)\right| \leq \varepsilon$ for any $x, x^{\prime} \in X$,

(2) $Y=B($ Image $(f), \varepsilon)$.

The Gromov-Hausdorff distance $d_{G H}(X, Y)$ between $X$ and $Y$ is defined by the infimum of those $\varepsilon>0$ so that there exist $\varepsilon$-approximations from $X$ to $Y$ and from $Y$ to $X$. We say that a sequence of metric spaces $X_{i}, i=1,2, \ldots$, converges to a metric space $X$ as $i \rightarrow \infty$ if $d_{G H}\left(X_{i}, X\right) \rightarrow 0$ as $i \rightarrow \infty$.

For two pointed metric spaces $(X, x),(Y, y)$, a pointed $\varepsilon$-approximation $f$ from $(X, x)$ to $(Y, y)$ is a map $f: B_{X}(x, 1 / \varepsilon) \rightarrow Y$ such that

(1) $f(x)=y$

(2) $\left|d\left(x^{\prime}, x^{\prime \prime}\right)-d\left(f\left(x^{\prime}\right), f\left(x^{\prime \prime}\right)\right)\right| \leq \varepsilon$ for $x^{\prime}, x^{\prime \prime} \in B_{X}(x, 1 / \varepsilon)$,

(3) $B_{Y}(y, 1 / \varepsilon) \subset B($ Image $(f), \varepsilon)$.

The pointed Gromov-Hausdorff distance $d_{G H}((X, x),(Y, y))$ between $(X, x)$ and $(Y, y)$ is defined by the infimum of those $\varepsilon>0$ so that there exist pointed $\varepsilon$ approximations from $(X, x)$ to $(Y, y)$ and from $(Y, y)$ to $(X, x)$.

For an $n$-dimensional Alexandrov space $X^{n}$, the (Gromov-Hausdorff) tangent cone $T_{x} X$ of $X$ at $x$ is defined by the pointed Gromov-Hausdorff limit of $\left(1 / r_{i} X, x\right)$ for some sequence $\left(r_{i}\right)$ converging to zero. Thus, $T_{x} X$ is an $n$-dimensional noncompact Alexandrov space with non-negative curvature. Also, $T_{x} X$ is isometric to the metric cone $K\left(\Sigma_{x}\right)$ over the space of directions $\Sigma_{x}$.

For a locally Lipschitz map $f: X \rightarrow M$ between Alexandrov spaces and a curve $\gamma:[0, a] \rightarrow X$ starting at $p=\gamma(0)$ with direction $\gamma^{+}$at $p$, we say that $f$ has the directional derivative $d f\left(\gamma^{+}\right)$in the direction $\gamma^{+}$if there exists the limit

$$
d f\left(\gamma^{+}\right):=(f \circ \gamma)^{+}:=\frac{d}{d t} f \circ \gamma(0+) .
$$

A distance function on an Alexandrov space has the directional derivative in any direction. 
For a local Lipschitz function $f$ on a metric space, the absolute gradient $|\nabla f|_{p}$ of $f$ at $p$ is defined by

$$
|\nabla f|_{p}:=|\nabla f|(p):=\max \left\{\limsup _{x \rightarrow p} \frac{f(x)-f(p)}{d(x, p)}, 0\right\} .
$$

Definition 2.12. $f$ is called regular at $p$ if $|\nabla f|_{p}>0$. Such a point $p$ is a regular point for $f$. Otherwise, $f$ is called critical at $p$.

Let $X$ be an Alexandrov space and $U$ be an open subset of $X$. Let $f: U \rightarrow \mathbb{R}$ be a locally Lipschitz function. For $\lambda \in \mathbb{R}, f$ is said to be $\lambda$-concave if for every segment $\gamma:[0, \ell] \rightarrow U$, the function

$$
f \circ \gamma(t)-\frac{\lambda}{2} t^{2}
$$

is concave in $t$. A 0 -concave function is said to be concave. $f$ is said to be semiconcave if for every $x \in U$ there are an open neighborhood $V$ of $x$ in $U$ and a constant $\lambda \in \mathbb{R}$ such that $\left.f\right|_{V}$ is $\lambda$-concave.

For a semiconcave function $f$ on a finite dimensional Alexandrov space, the gradient vector $\nabla f$ of $f$ is defined in the tangent cone:

Definition 2.13 ([PP QG $)$. Let $X$ be a finite dimensional Alexandrov space. Let $f: U \rightarrow \mathbb{R}$ be a semiconcave function defined on an open neighborhood $U$ of $p$. A vector $v \in T_{p} X$ is called the gradient of $f$ at $p$ if the following hold:

(i) For any $w \in T_{p} X$, we have $d_{p} f(w) \leq\langle v, w\rangle$.

(ii) $d_{p} f(v)=|v|^{2}$.

The gradient of $f$ at $p$ is denoted by $\nabla_{p} f$ for short.

Remark that $\nabla_{p} f$ is uniquely determined in the following manner: If $|\nabla f|_{p}=0$, then $\nabla_{p} f=o_{p}$, and otherwise

$$
\nabla_{p} f=d_{p} f\left(\xi_{\max }\right) \xi_{\max }
$$

where $\xi_{\max } \in \Sigma_{p}$ is the uniquely determined unit vector such that $d_{p} f\left(\xi_{\max }\right)=$ $\max _{\xi \in \Sigma_{p}} d_{p} f(\xi)$.

We can show that the absolute gradient $|\nabla f|(p)$ of $f$ is equal to the norm $\left|\nabla_{p} f\right|$ of gradient vector $\nabla_{p} f$ in $T_{p} X$.

2.4. Ultraconvergence. We will recall the notion of ultrafilters and ultralimits. For more details, we refer to $[\mathrm{BH}]$. A (non-principle) ultrafilter $\omega$ on the set of natural numbers $\mathbb{N}$ is a finitely additive measure on the power set $2^{\mathbb{N}}$ of $\mathbb{N}$ that has values 0 or 1 and contains no atoms. For each sequence $\left\{y_{i}\right\}=\left\{y_{i}\right\}_{i \in \mathbb{N}}$ in a compact Hausdorff space $Y$, an ultralimit $\lim _{\omega} y_{i}=y \in Y$ of this sequence is uniquely determined by the requirement $\omega\left(\left\{i \in \mathbb{N} \mid y_{i} \in U\right\}\right)=1$ for all neighborhoods $U$ of $y$. If $f: Y \rightarrow Z$ is a continuous map between topological spaces, then $\lim _{\omega} f\left(y_{i}\right)=f\left(\lim _{\omega} y_{i}\right)$.

For a sequence $\left\{\left(X_{i}, x_{i}\right)\right\}$ of pointed metric spaces, consider the set of all sequences $\left\{y_{i}\right\}$ of points $y_{i} \in X_{i}$ with $\lim _{\omega}\left|x_{i} y_{i}\right|<\infty$ and provide the pseudometric $\left|\left\{y_{i}\right\}\left\{z_{i}\right\}\right|=\lim _{\omega}\left|y_{i} z_{i}\right|$ on the set. The ultralimit $(X, x)=\lim _{\omega}\left(X_{i}, x_{i}\right)$ of $\left\{\left(X_{i}, x_{i}\right)\right\}$ is defined to be the metric space arising from this pseudometric, and the equivalence class of a sequence $\left\{y_{i}\right\}$ is denoted by $\left(y_{i}\right)$. The ultralimit of a constant sequence $\{(X, x)\}$ of a metric space $(X, x)$ is called the ultrapower of $(X, x)$ and is denoted by 
$X^{\omega}=\left(X^{\omega}, x\right)$. The natural map $X \ni y \mapsto(y)=(y, y, y, \ldots) \in X^{\omega}$ is an isometric embedding.

We review a relation between the ultraconvergence and the usual convergence. A sequence $\left(\varepsilon_{i}\right)$ of positive numbers is said to be a scale if $\lim _{i \rightarrow \infty} \varepsilon_{i}=0$.

Lemma 2.14. For a real number $A$ and a function $h: \mathbb{R}_{+} \rightarrow \mathbb{R}$, the following are equivalent:

(i) $\liminf _{t \searrow 0} h(t) \geq A$

(ii) For any scale $(o)=\left(t_{i}\right)$, we have $\lim _{\omega} h\left(t_{i}\right) \geq A$.

Proof. $((i) \Rightarrow(i i))$. We assume $(i)$. Then, for any $\varepsilon>0$, there is $t_{0}>0$ such that

$$
\inf _{0<t \leq t_{0}} h(t)>A-\varepsilon .
$$

Let us take any scale $\left(t_{i}\right)$. Then there is $i_{0}$ such that, for all $i \geq i_{0}$, we have

$$
h\left(t_{i}\right) \geq \inf _{0<t \leq t_{0}} h(t) .
$$

Therefore, taking an ultralimit, we have

$$
\lim _{\omega} h\left(t_{i}\right) \geq A-\varepsilon
$$

The above inequality holds for all $\varepsilon>0$. Then we obtain $(i i)$.

$((i i) \Rightarrow(i))$. We assume $(i i)$. We take a sequence $\left(t_{i}\right)$ tending to 0 such that

$$
\lim _{i \rightarrow \infty} h\left(t_{i}\right)=\liminf _{t \searrow 0} h(t) .
$$

Then, taking an ultralimit, we obtain $(i)$ :

$$
A \leq \lim _{\omega} h\left(t_{i}\right)=\lim _{i \rightarrow \infty} h\left(t_{i}\right)=\liminf _{t \searrow 0} h(t) .
$$

Let $\left(X_{i}, x_{i}\right)$ and $\left(Y_{i}, y_{i}\right)$ be sequences of pointed metric spaces and let $f_{i}$ : $\left(X_{i}, x_{i}\right) \rightarrow\left(Y_{i}, y_{i}\right)$ be a sequence of maps. Then the ultralimit $f_{\omega}=\lim _{\omega} f_{i}$ of $\left\{f_{i}\right\}$ is defined by

$$
\lim _{\omega} X_{i} \ni a_{\omega}=\left(a_{i}\right) \mapsto f_{\omega}\left(a_{\omega}\right):=\left(f_{i}\left(a_{i}\right)\right) \in \lim _{\omega} Y_{i}
$$

if it is well-defined. For instance, if $f_{i}$ is an $L_{i}$-Lipschitz map with $L_{\omega}:=\lim _{\omega} L_{i}<$ $\infty$, then the ultralimit $f_{\omega}$ is well-defined and $L_{\omega}$-Lipschitz. If $f_{i}:\left(X_{i}, x_{i}\right) \rightarrow\left(Y_{i}, y_{i}\right)$ is a pointed $\tau_{i}$-approximation with $\tau_{\omega}:=\lim _{\omega} \tau_{i}<\infty$, then the ultralimit $f_{\omega}$ is well-defined and a $\tau_{\omega}$-approximation. Remark that if $f_{i}:\left(X_{i}, x_{i}\right) \rightarrow\left(Y_{i}, y_{i}\right)$ and $g_{i}:\left(Y_{i}, y_{i}\right) \rightarrow\left(Z_{i}, z_{i}\right)$ have the ultralimits $f_{\omega}:=\lim _{\omega} f_{i}$ and $g_{\omega}:=\lim _{\omega} g_{i}$, then $\lim _{\omega}\left(g_{i} \circ f_{i}\right)=g_{\omega} \circ f_{\omega}$. For $a_{\omega}=\left(a_{i}\right), a_{\omega}^{\prime}=\left(a_{i}^{\prime}\right) \in \lim _{\omega} X_{i}$, we have $\left|f_{\omega}\left(a_{\omega}\right), f_{\omega}\left(a_{\omega}^{\prime}\right)\right|=\lim _{\omega}\left|f_{i}\left(a_{i}\right), f_{i}\left(a_{i}^{\prime}\right)\right|$.

For a pointed metric space $(X, x)$ and a scale $(o)=\left(\varepsilon_{i}\right)$, we define the blow-up $X_{x}^{(o)}=\left(X_{x}^{(o)}, o_{x}\right)$ of $(X, x)$ by

$$
\left(X_{x}^{(o)}, o_{x}\right):=\lim _{\omega}\left(1 / \varepsilon_{i} X, x\right) .
$$

For a map $f:(X, x) \rightarrow(Y, y)$ between pointed metric spaces, we consider a sequence $\left\{f_{i}\right\}$ of maps defined by

$$
f_{i}=f:\left(1 / \varepsilon_{i} X, x\right) \rightarrow\left(1 / \varepsilon_{i} Y, y\right) .
$$

The blow-up $f_{x}^{(o)}: X_{x}^{(o)} \rightarrow Y_{y}^{(o)}$ of $f$ is defined by $f_{x}^{(o)}:=\lim _{\omega} f_{i}$ if it is well-defined. 
Let $X$ be an Alexandrov space and $x \in X$, and let $(o)=\left(\varepsilon_{i}\right)$ be a scale. We consider the exponential map at $x$ :

$$
\exp _{x}:\left(\operatorname{dom}\left(\exp _{x}\right), o_{x}\right) \ni(\gamma, t) \mapsto \exp _{x}(\gamma, t):=\gamma(t) \in(X, x) .
$$

Here, $\operatorname{dom}\left(\exp _{x}\right) \subset T_{x} X$ is the domain of $\exp _{x}$. Since $\exp _{x}$ is locally Lipschitz, the blow-up of $\exp _{x}$ is well-defined and written by

$$
\exp _{x}^{(o)}:=\left(\exp _{x}\right)_{o_{x}}^{(o)}:\left(T_{x} X, o_{x}\right) \rightarrow\left(X_{x}^{(o)}, o_{x}\right)
$$

The domain of $\exp _{x}^{(o)}$ is the blow-up of $\left(\operatorname{dom}\left(\exp _{x}\right), o_{x}\right)$, which is identified as $\left(T_{x} X, o_{x}\right)$.

Lemma $2.15\left(\left[\mathrm{~L},[\mathrm{BGP})\right.\right.$. Let $(o)=\left(\varepsilon_{i}\right)$ be an arbitrary scale.

(i) Let $X$ be a (possibly infinite dimensional) Alexandrov space. Then $\exp _{x}^{(o)}$ is an isometric embedding.

(ii) If $X$ be a finite dimensional Alexandrov space. Then $\exp _{x}^{(o)}: K\left(\Sigma_{x}\right) \rightarrow X_{x}^{(o)}$ is surjective, for any $x \in X$.

Proof. (i) By the definition of the angle between geodesics, for any $(\gamma, s)$ and $(\eta, t) \in$ $\Sigma_{x}^{\prime} \times[0, \infty)$, we have

$$
\frac{\left|\gamma\left(s \varepsilon_{i}\right), \eta\left(t \varepsilon_{i}\right)\right|_{X}}{\varepsilon_{i}} \stackrel{i \rightarrow \infty}{\longrightarrow}|s \gamma, t \eta|_{K\left(\Sigma_{x}\right)} .
$$

(ii) By $\left[\mathrm{BGP}\right.$, the Gromov-Hausdorff tangent cone $T_{x} X$ and the cone $K\left(\Sigma_{x}\right)$ over a space of directions are isometric to each other. More precisely, the scaled logarithmic map

$$
\log _{x}=\exp _{x}^{-1}:\left(\frac{1}{\varepsilon_{i}} X, x\right) \rightarrow\left(\frac{1}{\varepsilon_{i}} T_{x} X, o_{x}\right)
$$

is a $\tau_{i}$-approximation for some sequence $\left\{\tau_{i}\right\}$ of positive numbers converging to zero, and $\exp _{x} \circ \log _{x}=i d$. Then we have, for each $\left(x_{i}\right) \in X_{x}^{(o)}$,

$$
\exp _{x}^{(o)}\left(\log _{x}\left(x_{i}\right)\right)=\left(\exp _{x} \circ \log _{x}\left(x_{i}\right)\right)=\left(x_{i}\right) .
$$

Therefore, $\exp _{x}^{(o)}$ is surjective.

2.5. Preliminaries from the geometry of Alexandrov spaces. In this subsection, we review the basic facts on the geometry and topology of Alexandrov spaces. We refer mainly to BGP, Per II].

2.5.1. Local structure around an almost regular point. Burago, Gromov and Perelman proved that a neighborhood of an almost regular point is almost isometric to an open subset of Euclidean space.

Theorem 2.16 ([BGP], $\mathrm{OS}]$ ). For $n \in \mathbb{N}$, there exists a positive number $\delta_{n}>0$ satisfying the following: Let $X$ be an $n$-dimensional Alexandrov space with curvature $\geq-1$. For $0<\delta \leq \delta_{n}$, if $x \in X$ is an $(n, \delta)$-strained point with a strainer $\left\{p_{\alpha}\right\}_{\alpha= \pm 1, \ldots, \pm n}$ of length $\ell$, then the two maps

$$
\begin{aligned}
\varphi & :=\left(d\left(p_{\alpha}, \cdot\right)\right)_{\alpha=1, \ldots, n} \\
\tilde{\varphi} & :=\left(\frac{1}{\mathcal{H}^{n}\left(B\left(p_{\alpha}, r\right)\right)} \int_{B\left(p_{\alpha}, \varepsilon\right)} d(y, \cdot) d \mathcal{H}^{n}(y)\right)_{\alpha=1, \ldots, n}
\end{aligned}
$$


on $B(x, r)$ for small $r>0$ are both $\left(\theta_{n}(\delta)+\theta_{n}(r / \ell)\right)$-almost isometries, where $\varepsilon$ is so small with $\varepsilon \ll r / \ell$. Here, $\theta_{n}(\delta)$ is a positive function depending on $n$ and $\delta$ such that $\lim _{\delta \rightarrow 0} \theta_{n}(\delta)=0$.

Lemma 2.17 ([Y conv Lemma 1.8]). Let $M$ be an $n$-dimensional Alexandrov space and $\delta$ be taken in Theorem 2.16. For any $(n, \delta)$-strained point $p \in M$, there exists $r>0$ satisfying the following: For every $q \in B(p, r / 2)$ and $\xi \in \Sigma_{q}$ there exists $x, y \in B(p, r)$ such that

$$
\begin{array}{r}
|x q|,|y q| \geq r / 4, \\
\left|x_{q}^{\prime}, \xi\right| \leq \theta(\delta, r), \\
\tilde{\angle} x q y \geq \pi-\theta(\delta, r) .
\end{array}
$$

Lemma 2.18 (Y conv, Lemma 1.9]). Let $M, p, r$ and $\delta$ be taken in Lemma 2.17. For every $q \in M$ with $r / 10 \leq|p q| \leq r$ and for every $x \in M$ with $|p x| \ll r$, we have

$$
|\angle x p q-\tilde{\angle} x p q|<\theta(\delta, r,|p x| / r) .
$$

2.5.2. Splitting Theorem. The Splitting Theorem is an important tool to study the structure of non-negatively curved spaces.

Theorem 2.19 (Splitting Theorem Milka]). Let $X$ be an Alexandrov space of curvature $\geq 0$. Suppose that there exists a line $\gamma: \mathbb{R} \rightarrow X$. Then there exists an Alexandrov space $Y$ of curvature $\geq 0$ such that $X$ is isometric to the product $Y \times \mathbb{R}$.

Theorem 2.20. If an Alexandrov space $\Sigma$ of curvature $\geq 1$ has the maximal diameter $\pi$, then $\Sigma$ is isometric to the metric suspension $\Sigma(\Lambda)$ over some Alexandrov space $\Lambda$ of curvature $\geq 1$.

Corollary 2.21. If an $n$-dimensional Alexandrov space $\Sigma$ of curvature $\geq 1$ has the maximal radius $\pi$, then $\Sigma$ is isometric to a unit $n$-sphere of constant curvature $=1$.

Remark 2.22 ( $\underline{\mathrm{M}}]$ ). The Splitting Theorem and Corollary 2.21 hold even for infinite dimensional Alexandrov spaces.

2.5.3. Convergence and collapsing theory. Yamaguchi proved the following two theorems (Theorems 2.24 and 2.25) for Alexandrov spaces converging to an almost regular Alexandrov space, which are counterparts of the Fibration Theorem Y91] in the Riemannian geometry.

Definition 2.23. A surjective map $f: X \rightarrow Y$ between Alexandrov spaces is called an $\varepsilon$-almost Lipschitz submersion if $f$ is an $\varepsilon$-approximation, and for any $x, y \in X$ setting $\theta:=\angle_{x}\left(y_{x}^{\prime}, \Sigma_{x} \Pi_{x}\right)$, we have

$$
\left|\frac{|f(x) f(y)|}{|x y|}-\sin \theta\right|<\varepsilon
$$

where $\Pi_{x}:=f^{-1}(f(x))$.

A surjective map $f: X \rightarrow Y$ is called an $\varepsilon$-almost isometry if for any $x, y \in X$ we have

$$
\left|\frac{|f(x) f(y)|}{|x y|}-1\right|<\varepsilon .
$$

Theorem 2.24 (Lipschitz submersion theorem [Y conv]). For $n \in \mathbb{N}$ and $\eta>0$, there exist $\delta_{n}, \varepsilon_{n}(\eta)>0$ satisfying the following. Let $M^{n}, X^{k}$ be Alexandrov spaces with curvature $\geq-1, \operatorname{dim} M^{n}=n$, and $\operatorname{dim} X^{k}=k$. Suppose that $\delta$-strain 
radius of $X>\eta$. Then if the Gromov-Hausdorff distance between $M$ and $X$ is less than $\varepsilon \leq \varepsilon_{n}(\eta)$, there is a $\theta(\delta, \varepsilon)$-almost Lipschitz submersion $f: M \rightarrow X$. Here, $\theta(\delta, \varepsilon)$ denotes a positive constant depending on $n, \eta$ and $\delta, \varepsilon$ and satisfying $\lim _{\delta, \varepsilon \rightarrow 0} \theta(\delta, \varepsilon)=0$.

When $M$ is almost regular (and $X$ has non-empty boundary), Theorem 2.24 deforms as Theorem 2.25 below. Let $X$ be a $k$-dimensional complete Alexandrov space with curvature $\geq-1$ having nonempty boundary. Let $X^{*}$ be another copy of $X$. Take the double $\operatorname{dbl}(X)=X \cup X^{*}$ of $X$. The double $\operatorname{dbl}(X)$ is also an Alexandrov space of curvature $\leq-1$. A $(k, \delta)$-strainer $\left\{\left(a_{i}, b_{i}\right)\right\}$ of $\operatorname{dbl}(X)$ at $p \in X$ is called admissible if $a_{i}, b_{j} \in X$ for $1 \leq i \leq k, 1 \leq j \leq k-1$ ( $b_{k}$ may be in $X^{*}$ if $p \in \partial X$ for instance). Let $R_{\delta}^{D}(X)$ be the set of all admissible $(k, \delta)$-strained points in $X$.

Let $Y$ be a closed domain of $R_{\delta}^{D}(X)$. For a small $\nu>0$, we put

$$
Y_{\nu}:=\{x \in Y \mid d(x, \partial X) \geq \nu\},
$$

and we put

$$
\partial_{0} Y_{\nu}:=Y_{\nu} \cap\left\{d_{\partial X}=\nu\right\}, \operatorname{int}_{0} Y_{\nu}:=Y_{\nu}-\partial_{0} Y_{\nu} .
$$

The admissible $\delta$-strained radius $\delta^{D}$-str.rad $x$ at $p \in X$ is the supremum of the length of all admissible $\delta$-strainers at $p$. The admissible $\delta$-strained radius $\delta^{D}$-str.rad $(Y)$ of a subset $Y \subset X$ is

$$
\delta^{D} \text {-str.rad }(Y):=\inf _{p \in Y} \delta^{D} \text {-str.rad } p .
$$

Theorem 2.25 (Fibration Theorem ( $\mathrm{Y}$ 4-dim, Theorem 1.2])). Given $k$ and $\mu>$ 0 , there exist positive numbers $\delta=\delta_{k}, \varepsilon_{k}(\mu)$ and $\nu=\nu_{k}(\mu)$ satisfying the following: Let $X^{k}$ be an Alexandrov space with curvature $\geq-1$ of dimension $k$. Let $Y \subset$ $R_{\delta}^{D}(X)$ be a closed domain such that $\delta_{D}$-str.rad $(Y) \geq \mu$. Let $M^{n}$ be an Alexandrov space with curvature $\geq-1$ of dimension $n$. Suppose that $R_{\delta_{n}}\left(M^{n}\right)=M^{n}$ for some small $\delta_{n}>0$. If $d_{G H}(M, X)<\varepsilon$ for some $\varepsilon \leq \varepsilon_{k}(\mu)$, then there exist a closed domain $N \subset M$ and a decomposition

$$
N=N_{\text {int }} \cup N_{\text {cap }}
$$

of $N$ into two closed domains glued along their boundaries and a Lipschitz map $f: N \rightarrow Y_{\nu}$ such that

(1) $N_{\text {int }}$ is the closure of $f^{-1}\left(\operatorname{int}_{0} Y_{\nu}\right)$ and $N_{\text {cap }}=f^{-1}\left(\partial_{0} Y_{\nu}\right)$;

(2) both the restrictions $f_{\mathrm{int}}:=\left.f\right|_{N_{\mathrm{int}}}: N_{\mathrm{int}} \rightarrow Y_{\nu}$ and $f_{\text {cap }}:=\left.f\right|_{N_{\text {cap }}}: N_{\text {cap }} \rightarrow$ $\partial_{0} Y_{\nu}$ are

(a) locally trivial fiber bundles (see Definition 2.37);

(b) $\theta(\delta, \nu, \varepsilon / \nu)$-Lipschitz submersions.

Remark 2.26. If $\partial X=\emptyset$, then $N_{\text {cap }}=\emptyset$ in the statement of Theorem 2.25.

The following theorem is a fundamental and important tool to study a local structure of collapsing Alexandrov spaces.

Theorem 2.27 (Rescaling Argument Y ess, SY00, Y Y 4-dim). Let $M_{i}, i=$ $1,2, \ldots$, be a sequence of Alexandrov spaces of dimension $n$ with curvature $\geq-1$ and let $X$ be an Alexandrov space of dimension $k$ with curvature $\geq-1$ and $k<n$. 
Let $p_{i} \in M_{i}$ and $p \in X$. Assume that $\left(M_{i}, p_{i}\right)$ converges to $(X, p)$, and $r>0$ is a small number depending on $p$. Assume the following:

Assumption 2.28. For any $\tilde{p}_{i}$ with $d\left(p_{i}, \tilde{p}_{i}\right) \rightarrow 0$ and for any sufficiently large $i$, $B\left(\tilde{p}_{i}, r\right)$ has a critical point for dist $_{\tilde{p}_{i}}$

Then there exist a sequence $\delta_{i} \rightarrow 0$ of positive numbers and $\hat{p}_{i} \in M_{i}$ such that

- $d\left(p_{i}, \hat{p}_{i}\right) \rightarrow 0$ as $i \rightarrow \infty$;

- for any limit $Y$ of $\left(\frac{1}{\delta_{i}} M_{i}, \hat{p}_{i}\right)$, we have $\operatorname{dim} Y \geq k+1$;

- $\operatorname{dim} S \leq \operatorname{dim} Y-\operatorname{dim} X$, where $S$ is a soul of $Y$.

Remark 2.29. If a sequence of $B\left(p_{i}, r\right)$ metric balls does not satisfy Assumption 2.28, then by the Stability Theorem 2.34 $B\left(\tilde{p}_{i}, r\right)$ (resp. $U\left(\tilde{p}_{i}, r\right)$ ) is homeomorphic to the closed cone $K_{1}\left(\Sigma_{\tilde{p}_{i}}\right)$ (resp. the open cone $K\left(\Sigma_{\tilde{p}_{i}}\right)$ ) over the space of directions $\Sigma_{\tilde{p_{i}}}$ for some $\tilde{p}_{i} \in M_{i}$ with $d\left(p_{i}, \tilde{p}_{i}\right)$ tending to zero.

Fukaya and Yamaguchi proved the following.

Theorem 2.30 ([FY], Y $\mathrm{Y}$ conv $]$ ). For $n \in \mathbb{N}$, there exists $\varepsilon_{n}>0$ satisfying the following. Suppose that an $n$-dimensional Alexandrov space $M^{n}$ with curvature $\geq-1$ and diam $M^{n}<\varepsilon_{n}$. Then, the fundamental group $\pi_{1}\left(M^{n}\right)$ is almost nilpotent; i.e. $\pi_{1}\left(M^{n}\right)$ has a nilpotent subgroup of finite index.

Remark 2.31 (Y conv ). In Fibration Theorems 2.24 and 2.25 the fiber is connected and has an almost nilpotent fundamental group.

2.5.4. Perelman's Morse theory and stability theorem. In this section, we mainly refer to [Per II].

Definition 2.32 ([Per II] $)$. Let $f=\left(f_{1}, \ldots f_{m}\right): U \rightarrow \mathbb{R}^{m}$ be a map on an open subset $U$ of an Alexandrov space $X$ defined by $f_{i}=d\left(A_{i}, \cdot\right)$ for compact subsets $A_{i} \subset X$. The map $f$ is said to be $(c, \varepsilon)$-regular at $p \in U$ if there is a point $w \in X$ such that:

(1) $\angle\left(\left(A_{i}\right)_{p}^{\prime},\left(A_{j}\right)_{p}^{\prime}\right)>\pi / 2-\varepsilon$.

(2) $\angle\left(w_{p}^{\prime},\left(A_{i}\right)_{p}^{\prime}\right)>\pi / 2+c$.

Theorem 2.33 ([Per II] $)$. Let $X$ be an finite dimensional Alexandrov space, let $U \subset X$ be an open subset, and let $f$ be $(c, \varepsilon)$-regular at each point of $U$. If $\varepsilon$ is small compared with $c$, then we have:

(1) $f$ is a topological submersion (see Definition 2.37).

(2) If $f$ is proper in addition, then the fibers of $f$ are MCS-spaces. Hence $f$ is a fiber bundle over its image.

Here, a metrizable space $X$ is called an $n$-dimensional $M C S$-space if any point $p \in X$ has an open neighborhood $U$ and there exists an $(n-1)$-dimensional compact MCS-space $\Sigma$ such that $(U, p)$ is a pointed homeomorphic to the cone $(K(\Sigma), o)$, where $o$ is the apex of the cone. Here, we regarded the $(-1)$-dimensional MCS-space as the empty-set and its cone as the single-point set.

Perelman proved the Stability Theorem:

Theorem 2.34 (Stability Theorem Per II] (cf. [Kap Stab)). Let $X^{n}$ be a compact $n$-dimensional Alexandrov space with curvature $\geq \kappa$. Then there exists $\delta>0$ depending on $X$ such that if $Y^{n}$ is an $n$-dimensional Alexandrov space with curvature $\geq \kappa$ and $d_{G H}(X, Y)<\delta$, then $Y$ is homeomorphic to $X$. 
In addition, let $A \subset X$ be a compact subset, and let $A^{\prime} \subset Y$ be a compact subset. Then there exists $\delta>0$ depending $(X, A)$ satisfying the following. Suppose that there is a $\delta$-approximation $f: Y \rightarrow X$ such that $f\left(A^{\prime}\right) \subset A$ and $\left.f\right|_{A^{\prime}}$ is a $\delta$-approximation. If $t \in\left(0, \sup d_{A}\right)$ is a regular value of $d_{A}$, then $S(A, t)$ is homeomorphic to $S\left(A^{\prime}, t\right)$. Here, we say that $t$ is a regular value if $d_{A}$ is regular on $S(A, t)$.

In particular, every point in a finite dimensional Alexandrov space has a cone neighborhood over its spaces of directions.

Theorem 2.35 ([Per II $]$ ). If an $n$-dimensional Alexandrov space $\Sigma^{n}$ of curvature $\geq 1$ has diameter greater than $\pi / 2$, then $\Sigma$ is homeomorphic to a suspension over an $(n-1)$-dimensional Alexandrov space of curvature $\geq 1$.

Theorem 2.36 ([Per II], Pet Appl], [GP]). If an $n$-dimensional Alexandrov space $\Sigma^{n}$ of curvature $\geq 1$ has radius $>\pi / 2$, then $\Sigma$ is homeomorphic to an $n$-sphere.

2.5.5. Preliminaries from Siebenmann's theory in [Sie].

Definition 2.37. A continuous map $p: E \rightarrow X$ between topological spaces is called a topological submersion (or called a locally trivial fiber bundle) if for any $y \in E$ there are an open neighborhood $U$ of $y$ in the fiber $p^{-1}(p(y))$, an open neighborhood $N$ of $p(y)$ in $X$, and an open embedding $f: U \times N \rightarrow E$ such that $p \circ f$ is the projection $U \times N \rightarrow N$. We call the embedding $f: U \times N \rightarrow E$ a product chart about $U$ for $p$, and the image $f(U \times N)$ a product neighborhood around $y$.

A surjective continuous map $p: E \rightarrow X$ of topological spaces is called a topological fiber bundle if there exists an open covering $\left\{U_{\alpha}\right\}$ of $X$, a family $\left\{F_{\alpha}\right\}$ of topological spaces, and a family $\left\{\varphi_{\alpha}: p^{-1}\left(U_{\alpha}\right) \rightarrow U_{\alpha} \times F_{\alpha}\right\}$ of homeomorphisms such that $\operatorname{proj}_{U_{\alpha}} \circ \varphi_{\alpha}=\left.p\right|_{p^{-1}\left(U_{\alpha}\right)}$ holds for each $\alpha$. Here, $\operatorname{proj}_{U_{\alpha}}$ is the projection from $U_{\alpha} \times F_{\alpha}$ to $U_{\alpha}$.

A finite dimensional topological space $Y$ is said to be a $W C S$-set [Sie, $\S 5]$ if it satisfies both (1) and (2):

(1) $Y$ is stratified into topological manifolds; i.e. it has a stratification

$$
Y \supset \cdots \supset Y^{(n)} \supset Y^{(n-1)} \supset \cdots \supset Y^{(-1)}=\emptyset,
$$

such that $Y^{(n)}-Y^{(n-1)}$ is a topological $n$-manifold without boundary.

(2) For each $x \in Y^{(n)}-Y^{(n-1)}$ there are a cone $C$ with a vertex $v$ and a homeomorphism $\rho: \mathbb{R}^{n} \times C \rightarrow Y$ onto an open neighborhood of $x$ in $Y$ such that $\rho^{-1}\left(Y^{(n)}\right)=\mathbb{R}^{n} \times\{v\}$.

From the definition, we can see that an MCS-space is a WCS-set.

Theorem 2.38 (Union Lemma [Sie]). Let $p: E \rightarrow X$ be a topological submersion and $F=p^{-1}\left(x_{0}\right)$ the fiber over $x_{0} \in X$. We assume that $F$ is a WCS-space. Let $A_{1}$ and $A_{2}$ be compact sets in $F$. Let $\varphi_{i}: U_{i} \times N_{i} \rightarrow E$ be a product chart about $U_{i}$ for an open neighborhood $U_{i}$ of $A_{i}$ in $F$, and $i=1,2$. Then there exists a product chart $\varphi: U \times N \rightarrow E$ about $U \supset A_{1} \cup A_{2}$ in $F$ such that

$$
\varphi=\left\{\begin{array}{l}
\varphi_{1} \text { near } A_{1} \times\left\{x_{0}\right\}, \\
\varphi_{2} \text { near }\left(A_{2}-U_{1}\right) \times\left\{x_{0}\right\} .
\end{array}\right.
$$

Theorem 2.39 ([Sie]). Let $p: E \rightarrow X$ be a topological submersion. We assume that $p$ is proper and all fibers of $p$ are WCS-spaces. Then $p$ is a topological fiber bundle over $p(E)$. 
We provide the following lemma that will be used in Section 5

Lemma 2.40. Let $f: E \rightarrow[0,1]$ be a fiber bundle and the fiber $F:=f^{-1}(0)$ be a $W C S$-space. Let $U \subset F$ be an open subset and $A \subset U$ be a closed subset. Suppose that $\varphi: U \times[0,1] \rightarrow E$ is a product chart about $U$ for $f$. Then there exists a product chart $\chi: F \times[0,1] \rightarrow E$ such that

$$
\chi=\varphi \text { on } A \times[0,1] .
$$

In particular, $E-\varphi(A \times[0,1])$ is homeomorphic to $(F-A) \times[0,1]$.

Proof. We may assume that $E=F \times[0,1]$ and $f$ is the projection onto $[0,1]$. Let $\varphi: U \times[0,1] \rightarrow F \times[0,1]$ be a product chart about $U$. Using Union Lemma 2.38 and the compactness of $[0,1]$, we will construct an extension of $\left.\varphi\right|_{A \times[0,1]}$ to a product chart defined on $F \times[0,1]$.

By Union Lemma 2.38, for any $t \in[0,1]$, there exist an open neighborhood $N_{t}$ of $t$ in $[0,1]$ and a product chart

$$
\psi^{(t)}: F \times N_{t} \rightarrow E
$$

such that

$$
\left.\psi^{(t)}\right|_{A \times N_{t}}=\left.\varphi\right|_{A \times N_{t}} .
$$

By the Lebesgue number lemma, there is $n \in \mathbb{N}$ such that, setting $I_{k}:=[k / n$, $(k+1) / n],\left\{I_{k}\right\}_{k=0,1, \ldots, n-1}$ is a refinement of an open covering $\left\{N_{t}\right\}_{t \in[0,1]}$ of $[0,1]$. Namely, for $k=0,1, \ldots, n-1$, there is $t_{k} \in[0,1]$ such that $I_{k} \subset N_{t_{k}}$. Let us set

$$
\psi^{k}:=\left.\psi^{\left(t_{k}\right)}\right|_{F \times I_{k}} .
$$

For $t \in I_{k}$, let us define a homeomorphism $\psi_{t}^{k}: F \rightarrow F$ by the equality

$$
\psi^{k}(x, t)=\left(\psi_{t}^{k}(x), t\right) .
$$

Gluing these local product charts $\psi^{k}$, we construct the required product chart $\chi$ as follows. We inductively define a homeomorphism $\chi_{t}^{k}: F \rightarrow F$ by

$$
\begin{array}{ll}
\chi_{t}^{0}=\psi_{t}^{0} & \text { for } t \in I_{0}, \\
\chi_{t}^{k}=\psi_{t}^{k} \circ\left(\psi_{k / n}^{k}\right)^{-1} \circ \chi_{k / n}^{k-1} & \text { for } t \in I_{k}, k \geq 1 .
\end{array}
$$

For $k=0,1, \ldots, n-1$ and $(x, t) \in F \times I_{k}$, we define

$$
\chi(x, t):=\left(\chi_{t}^{k}(x), t\right) \text {. }
$$

One can easily check that

$$
\chi=\varphi \text { on } A \times[0,1] .
$$

Namely, $\chi: F \times[0,1] \rightarrow E$ satisfies the conclusion of the lemma.

2.6. Differentiable structures of Alexandrov spaces. Otsu and Shioya OS proved that any Alexandrov space has a differential structure and a Riemannian structure in a weak sense.

Definition 2.41 ([Per DC] $)$. Let $U \subset M^{n}$ be an open subset of an Alexandrov space $M$. A locally Lipschitz function $f: U \rightarrow \mathbb{R}$ is called a $D C$-function if for any $x \in U$ there exist two (semi-)concave functions $g$ and $h$ on some neighborhood $V$ of $x$ in $U$ such that $f=g-h$ on $V$. A locally Lipschitz map $f=\left(f_{1}, \ldots, f_{m}\right): U \rightarrow \mathbb{R}^{m}$ is called a $D C$-map if each $f_{i}$ is a $D C$-function.

In [KMS, §2.6], the authors formulated a general concept of structure on topological spaces. 
Definition 2.42 ([KMS $]$ ). For an integer $n \geq 0$, we consider the family

$$
\mathcal{F}=\left\{\mathcal{F}(U ; A) \mid U \subset \mathbb{R}^{n} \text { is an open subset and } A \subset U \text { a subset }\right\}
$$

such that

(i) each $\mathcal{F}(U ; A)$ is a class of maps from $U$ to $\mathbb{R}^{n}$;

(ii) if $A \supset B$, then $\mathcal{F}(U ; A) \subset \mathcal{F}(U ; B)$;

(iii) if $f \in \mathcal{F}(U ; A), g \in \mathcal{F}(V ; B)$, and $f(U) \subset V$, then

$$
g \circ f \in \mathcal{F}\left(U ; A \cap f^{-1}(B)\right) .
$$

The following are examples of $\mathcal{F}=\{\mathcal{F}(U ; A)\}$.

(Class $\left.C^{1}\right)$ Let $C^{1}(U ; A)$ be the class of maps from $U$ to $\mathbb{R}^{n}$ which are $C^{1}$ on $A$; i.e. they are differentiable on $A$ and their derivatives are continuous on $A$.

(Class $D C)$ Let $D C(U ; A)$ be the class of maps from $U$ to $\mathbb{R}^{n}$ which are $D C$ on some open subset $O \subset \mathbb{R}^{n}$ with $A \subset O \subset U$.

Let $X$ be a paracompact Hausdorff space, $Y \subset X$ a subset, and $\mathcal{F}$ as above. We call a pair $(U, \varphi)$ a local chart of $X$ if $U$ is an open subset of $X$ and if $\varphi$ is a homeomorphism from $U$ to an open subset of $\mathbb{R}^{n}$. A family $\mathcal{A}=\{(U, \varphi)\}$ of local charts of $X$ is called an $\mathcal{F}$-atlas on $Y \subset X$ if the following (i) and (ii) hold:

(i) $Y \subset \bigcup_{(U, \varphi) \in \mathcal{A}} U$.

(ii) If two local charts $(U, \varphi),(V, \psi) \in \mathcal{A}$ satisfy $U \cap V \neq \emptyset$, then

$$
\psi \circ \varphi^{-1} \in \mathcal{F}(\varphi(U \cap V) ; \varphi(U \cap V \cap Y)) .
$$

Two $\mathcal{F}$-atlases $\mathcal{A}$ and $\mathcal{A}^{\prime}$ on $Y \subset X$ are said to be equivalent if $\mathcal{A} \cup \mathcal{A}^{\prime}$ is also an $\mathcal{F}$-atlas on $Y \subset X$. We call each equivalent class of $\mathcal{F}$-atlases on $Y \subset X$ an $\mathcal{F}$-structure on $Y \subset X$.

Assume that $Y=X$. Then, an $\mathcal{F}$-structure on $Y \subset X$ is simply called an $\mathcal{F}$ structure on $X$. If there is an $\mathcal{F}$-structure on $X$, then $X$ is a topological manifold. We call a space equipped with an $\mathcal{F}$-structure an $\mathcal{F}$-manifold. Notice that $\mathcal{F}$ manifolds for $\mathcal{F}=C^{1}$ are nothing more than $C^{1}$-differentiable manifolds in the usual sense.

Let $M^{n}$ be an $n$-dimensional Alexandrov space. Fix a number $\delta>0$ with $\delta \ll 1 / n$. By Theorem 2.16, for any $x \in M-S_{\delta}(M)$, we obtain a local chart $(U, \tilde{\varphi})$, $U=U(x, r)$. The family $\mathcal{A}_{0}$ of all the $(U, \tilde{\varphi})$ 's on $M$ induces:

Theorem 2.43 ([OS]). There exists a $C^{1}$-structure on $M-S(M) \subset M$ containing $\mathcal{A}_{0}$.

Theorem 2.44 ([Per DC] $)$. There exists a DC-structure on $M-S_{\delta}(M) \subset M$ containing $\mathcal{A}_{0}$.

Thus, $M-S_{\delta}(M)$ is a $D C^{1}$-manifold with singular set $S(M)$ in the following sense.

Definition 2.45 ([KMS, §5]). A paracompact topological manifold $V$ with a subset $S \subset V$ is said to be a $D C^{1}$-manifold with singular set $S$ if $V$ possesses a $D C$-atlas $\mathcal{A}$ on $V$ which is also a $C^{1}$-atlas on $V-S \subset V$. We say that each local chart compatible with the atlas $\mathcal{A}$ is a $D C^{1}$-local chart.

Let $V^{\prime}$ be an another $D C^{1}$-manifold with singular set $S^{\prime}$. A map $f: V \rightarrow V^{\prime}$ is called a $D C^{1}$-map if for any $D C^{1}$-local chart $\left(U^{\prime}, \varphi^{\prime}\right)$ of $V^{\prime},\left(f^{-1}\left(U^{\prime}\right), \varphi^{\prime} \circ f\right)$ is a $D C^{1}$-local chart of $V$. A homeomorphism $f: V \rightarrow V^{\prime}$ is called a $D C^{1}$ homeomorphism if $f$ and $f^{-1}$ are $D C^{1}$-maps. 
Using Otsu's method [O], Kuwae, Machigashira and Shioya [KMS proved that an almost regular Alexandrov space has a smooth approximation by a Riemannian manifold.

Theorem 2.46 ( $[\mathrm{KMS}$; cf. $\mathrm{O}]$ ). For any $n \in \mathbb{N}$, there exists a positive number $\varepsilon_{n}>0$ depending only on $n$ satisfying the following: If $C$ is a compact subset in an $n$-dimensional Alexandrov space $M$ with curvature $\geq-1$ and it is $\varepsilon$-strained for $\varepsilon \leq \varepsilon_{n}$, then there exist an open neighborhood $U(C)$, a $C^{\infty}$-Riemannian $n$ manifold $N(C)$ with $C^{\infty}$-Riemannian metric $g_{N(C)}$, and a $\theta(\varepsilon)$-isometric $D C^{1}$ homeomorphism $f: U(C) \rightarrow N(C)$ such that $g_{N(C)}(d f(v), d f(w))=\langle v, w\rangle+\theta(\varepsilon)$ for any $v, w \in \Sigma_{x} U(C)$ and $x \in U(C)$. Here, $\langle\cdot, \cdot\rangle$ is the inner product of $T_{x} M$.

Remark 2.47. Otsu [O] proved this theorem for any Riemannian manifold $M$ with a lower sectional curvature bound and having small excess.

We will review the proof of Theorem 2.46 in the proof of Theorem 3.2 in Section3. It is important and needed in our proof of Theorem 3.2 .

2.7. Generalized Seifert fiber spaces. To describe results obtained in the present paper we define the notion of a generalized Seifert fiber space.

Definition 2.48. Let $M^{3}$ and $X^{2}$ be, respectively, a three-dimensional and a two-dimensional topological orbifold possibly with boundaries. A continuous map $f: M \rightarrow X$ is called a generalized Seifert fibration if there exists a family $\left\{c_{x}\right\}_{x \in X}$ of subsets of $M$ such that the following properties hold:

- The index set of $\left\{c_{x}\right\}$ is $X$. Each $x \in X, f^{-1}(x)=c_{x}$.

- Each $c_{x}$ is homeomorphic to a circle or a bounded closed interval. $c_{x}$ are disjoint and

$$
\bigcup_{x \in X} c_{x}=M
$$

- For each $x \in X$, there exists a closed neighborhood $U_{x}$ of $x$ such that $U_{x}$ is homeomorphic to a disk, and putting $V_{x}:=f^{-1}\left(U_{x}\right), V_{x}$ satisfies the following.

(i) If $c_{x}$ is topologically a circle, then $\left.f\right|_{V_{x}}: V_{x} \rightarrow U_{x}$ is a Seifert fibered solid torus in the usual sense.

(ii) If $c_{x}$ is topologically a bounded closed interval, then there exist homeomorphisms $\tilde{\phi}_{x}: V_{x} \rightarrow B(\mathrm{pt})$ and $\phi_{x}: U_{x} \rightarrow K_{1}\left(S_{\pi}^{1}\right)$, which preserve the structure of circle fibration with singular fiber. Namely, the following diagram commutes:

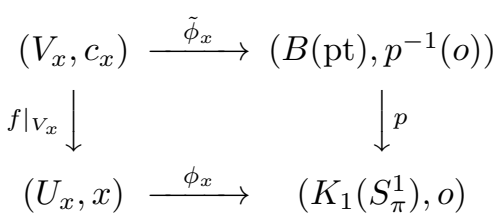

Here, $B(\mathrm{pt})=S^{1} \times D^{2} / \mathbb{Z}_{2}$ is the topological orbifold defined after Theorem 1.7 and $p$ is a canonical projection.

- If $\partial X$ has a compact component $C$, then there is a collar neighborhood $N$ of $C$ in $X$ such that $\left.f\right|_{f^{-1}(N)}$ is a usual circle fiber bundle over $N$.

We say that a three-dimensional topological orbifold $M$ is a generalized Seifert fiber space over $X$ if there exists a generalized Seifert fibration $f: M \rightarrow X$. Each 
fiber $f^{-1}(x)=c_{x}$ of $f$ is often called an orbit of $M$. An orbit $c_{x}$ is called singular if $V_{x}$ is a usual Seifert solid torus of $(\mu, \nu)$-type with $\mu>1$ or if $c_{x}$ is homeomorphic to an interval.

2.8. Soul Theorem from SY00] with complete classification. In this subsection, we recall the Soul Theorem for open three-dimensional Alexandrov spaces of non-negative curvature, obtained in SY00. Also, we classify the geometry and topology of open three-dimensional Alexandrov spaces of non-negative curvature having two-dimensional soul together with some new precise arguments. The Soul Theorem is very important to determine the topology of a neighborhood around a singular point in a collapsing three-dimensional Alexandrov space.

Definition 2.49. Let $M^{n}$ be an $n$-dimensional non-compact Alexandrov space with non-negative curvature. For a ray $\gamma:[0, \infty) \rightarrow M$ in $M$, we define the Busemann function $b_{\gamma}: M \rightarrow \mathbb{R}$ with respect to $\gamma$ as follows:

$$
b_{\gamma}(x):=\lim _{t \rightarrow \infty} d(\gamma(t), x)-t
$$

for $x \in M$. Fix a point $p \in M$ and define the Busemann function $b: M \rightarrow \mathbb{R}$ with respect to $p$ by

$$
b(x):=\inf _{\gamma} b_{\gamma}(x)
$$

for $x \in M$. Here, $\gamma$ runs over all the rays emanating from $p$. The Busemann functions $b_{\gamma}$ and $b$ are concave on $M$.

We denote by $C(0)$ the set of all points attaining the maximum value of $b$ :

$$
C(0):=b^{-1}\left(\max _{M} b\right) \text {. }
$$

Since $b$ is concave, $C(0)$ is an Alexandrov space possibly with boundary of dimension less than $n$. If $C(0)$ has no boundary, we call it a soul of $M$. Inductively, if $C(k)$, $k \geq 0$, has the non-empty boundary, we define $C(k+1)$, the set of all points attaining the maximum value of the distance function dist $_{\partial C(k)}$ from the boundary $\partial C(k)$ :

$$
C(k+1):=\operatorname{dist}_{\partial C(k)}^{-1}\left(\max _{C(k)} \operatorname{dist}_{\partial C(k)}\right) .
$$

Since dist ${ }_{\partial C(k)}$ is concave on $C(k), C(k+1)$ is also an Alexandrov space of dimension $<\operatorname{dim} C(k)$. Since $M$ has finite dimension, this construction stops, i.e. $\partial C(k)=\emptyset$ for some $k \geq 0$. Then we call such $C(k)$ a soul of $M$.

Proposition 2.50 ([Per II]; cf. Pet Semi, §2]). For any open Alexandrov space $M$ of non-negative curvature and its soul $S$, there is a Sharafutdinov retraction from $M$ to $S$. In particular, $S$ is homotopic to $M$.

2.8.1. Soul Theorem. We recall that a non-compact Alexandrov space without boundary is called open. In this section, we state the Soul Theorem for open threedimensional Alexandrov spaces of non-negative curvature obtained in [SY00]. We also define examples of open three-dimensional Alexandrov spaces of non-negative curvature which are not topological manifolds and study those topologies.

First, we shall prove a rigidity result for the case that a soul has codimension one. This is a generalization of [SY00, Theorem 9.8(2)].

Theorem 2.51. Let $M$ be an $n$-dimensional open Alexandrov space and let $S$ be a soul of $M$. Suppose that $\operatorname{dim} S=n-1$ and $S$ has a one-normal point. Let $B=B(S, t)$ be a metric ball around $S$ of radius $t>0$. Then, the metric sphere 
$\hat{S}:=\partial B$ equipped with the induced intrinsic metric is an Alexandrov space of nonnegative curvature. Also, $\hat{S}$ has an isometric involution $\sigma$ such that $\hat{S} / \sigma$ is isometric to $S$ and $M$ is isometric to $\hat{S} \times \mathbb{R} /(x, t) \sim(\sigma(x),-t)$.

Proof. Let us denote by

$$
\pi: M \rightarrow S
$$

a canonical projection. Namely, for $x \in M$, we set $\pi(x) \in S$ to be the nearest point from $x$ in $S$. We use rigidity facts on the $\pi$, referring to [SY00, §9] and [Y 4-dim, §2], for proving the theorem.

Assertion 2.52. $\hat{S}$ satisfies the following convexity property: For $x, y \in \hat{S}$ with $|x y|<2 t$, any geodesic $\gamma$ between $x$ and $y$ in $M$ is contained in $\hat{S}$. In particular, $\hat{S}$ with the induced intrinsic metric is an Alexandrov space of non-negative curvature.

Proof of Assertion 2.52. Since $|x y|<2 t, \gamma$ does not intersect $S$. From the total convexity of $B$, we have $\gamma \subset B$. Let us consider a curve $\bar{\gamma}:=\pi \circ \gamma$ on $S$. Let $\sigma_{s}$ denote a unique ray emanating from $\bar{\gamma}(s)$ containing $\gamma(s)$. By Y 4-dim, Proposition 2.1],

$$
\Pi:=\bigcup_{s \in[0,|x y|]} \sigma_{s}
$$

is a flatly immersed surface in $M$. Moving $\gamma$ along with $\Pi$, we obtain a curve $\hat{\gamma}$ contained in $\hat{S}$. This is a lift of $\bar{\gamma}$ via $\pi: \hat{S} \rightarrow S$. Therefore, we obtain $L(\hat{\gamma})=$ $L(\bar{\gamma}) \leq L(\gamma)=|x y|$. Suppose that $\gamma$ is not contained in $\hat{S}$. From the construction of $\hat{\gamma}$ and [Y 4-dim, Proposition 2.1], one can show that $L(\hat{\gamma})<L(\gamma)$. This is a contradiction. Therefore, $\hat{\gamma}$ must coincide with $\gamma$.

Now, we denote by $\hat{d}$ the induced intrinsic metric on $\hat{S}$. Assertion 2.52 says that $(\hat{S}, \hat{d})$ is an Alexandrov space of non-negative curvature. Let us denote by $\hat{\pi}: \hat{S} \rightarrow S$ the restriction of $\pi$ on $\hat{S}$. Let $S_{\text {two }}$ (resp. $S_{\text {one }}$ ) denote the set of all two-normal (resp. one-normal) points in $S$. We set $\hat{S}_{\text {two }}:=\hat{\pi}^{-1}\left(S_{\text {two }}\right)$ and $\hat{S}_{\text {one }}:=\hat{\pi}^{-1}\left(S_{\text {one }}\right)$. Then, $\hat{\pi}: \hat{S}_{\text {two }} \rightarrow S_{\text {two }}$ is a two-to-one map, and $\hat{\pi}: \hat{S}_{\text {one }} \rightarrow S_{\text {one }}$ is a one-to-one map.

Let us consider $S_{\text {reg }}:=S \cap M_{\delta}^{\text {reg }}$ for a small $\delta>0$, which is open dense in $S$. Note that since any one-normal point is an essentially singular point [SY00, $S_{\text {reg }}$ is contained in $S_{\text {two }}$. By Pet Para, $S_{\text {reg }}$ is convex, and hence, it is connected. We set $\hat{S}_{\text {reg }}:=\hat{\pi}^{-1}\left(S_{\text {reg }}\right)$. The restriction

$$
\hat{\pi}: \hat{S}_{\text {reg }} \rightarrow S_{\text {reg }}
$$

is a double covering. We define an involution $\sigma$ on $\hat{S}_{\text {reg }}$ as the non-trivial deck transformation of $\hat{\pi}: \hat{S}_{\text {reg }} \rightarrow S_{\text {reg. }}$. By using [Y 4-dim, Proposition 2.1], we conclude that $\sigma$ is a local isometry. Hence, there is a continuous extension of $\sigma$ on the whole $\hat{S}$. We denote it by the same notation $\sigma$. Then, $\sigma$ on $(\hat{S}, \hat{d})$ is also a local isometric involution. We note that $\sigma$ on $\hat{S}_{\text {one }}$ is defined as the identity. From the construction, $\sigma$ is bijective. Therefore, $\sigma$ is an isometry on $\hat{S}$ with respect to $\hat{d}$. We now fix the metric $\hat{d}$ on $\hat{S}$. By construction, $\hat{S} / \sigma$ and $S$ are isometric to each other.

Let us consider the quotient space $N:=\hat{S} \times \mathbb{R} /(x, s) \sim(\sigma(x),-s)$, which is an open Alexandrov space of non-negative curvature. We define $\varphi: N \rightarrow M$ as sending $[x, t] \in N$ to $x \in \hat{S}$. By construction, $\varphi$ is an isometry. 
Example 2.53 ([SY00, p. 39]). For a non-negatively curved closed Alexandrov surface $S$ and $p_{1}, p_{2} \ldots, p_{k} \in S\left(k \in \mathbb{Z}_{\geq 0}\right)$, we denote by $L(S ; k)=L\left(S ; p_{1}, p_{2} \ldots, p_{k}\right)$ an open three-dimensional Alexandrov space of non-negative curvature (if it exists) satisfying the following:

(1) $p_{1}, p_{2}, \ldots, p_{k}$ are essential singular points in $S$, and $S$ is isometric to a soul of $L(S ; k)$. Hereafter, $S$ is identified as a soul of $L(S ; k)$.

(2) $\left\{p_{1}, \ldots, p_{k}\right\}$ is the set of all topological singular points in $L(S ; k)$.

(3) There is a continuous surjection $\pi: L(S ; k) \rightarrow S$ such that for $x \in$ $S-\left\{p_{1}, \ldots, p_{k}\right\}, \pi^{-1}(x)$ is the union of two rays emanating from $x$ perpendicular to $S$; and for $x \in\left\{p_{1}, \ldots, p_{k}\right\}, \pi^{-1}(x)$ is the unique ray emanating from $x$ perpendicular to $S$.

(4) The restriction $\pi: \pi^{-1}\left(S-\left\{p_{1}, \ldots, p_{k}\right\}\right) \rightarrow S-\left\{p_{1}, \ldots, p_{k}\right\}$ is a line bundle.

Proposition 2.54 ([SY00, Proposition 9.5]; cf. Y 4 -dim, $\$ 17]$ ). If $k \geq 1$, then any space $L(S ; k)$ is one of $L\left(S^{2} ; 2\right), L\left(P^{2} ; 2\right)$ and $L\left(S^{2} ; 4\right)$.

Remark 2.55. There is an error in Proposition 9.5 (and Theorem 9.6) in [SY00]. Actually, a space $L(S ; 1)$ cannot exist, and a space $L(S ; 2)$ can have a soul homeomorphic to $P^{2}$. See [Y 4-dim, $\left.\S 17\right]$.

Proof of Proposition 2.54. Since $k \geq 1$, by Theorem 2.68, $S$ is homeomorphic to $S^{2}$ or $P^{2}$. Moreover, if $S \approx S^{2}$, then we have $k \leq 4$; and if $S \approx P^{2}$, then $k \leq 2$.

We consider the case that $S \approx P^{2}$. Suppose that $k=1$. Let $p \in S$ be a unique topological singular point in $L(S ; 1)$. Let $\pi: L(S ; 1) \rightarrow S$ be a surjection obtained in Example 2.53. For a neighborhood $B$ of $p$ in $S$ homeomorphic to $D^{2}$, the restriction

$$
\pi: \pi^{-1}(B) \rightarrow B
$$

is fiber-wise isomorphic to $\pi_{0}: D^{2} \times \mathbb{R} / \mathbb{Z}_{2} \rightarrow D^{2} / \mathbb{Z}_{2}$ such that $p \in B$ corresponds to the origin of $D^{2} / \mathbb{Z}_{2}$. Here, $D^{2} \times \mathbb{R} / \mathbb{Z}_{2}$ denotes the quotient space of $D^{2} \times \mathbb{R}$ by an involution $(x, t) \mapsto(-x,-t), D^{2} / \mathbb{Z}_{2}$ denotes the quotient space of $D^{2}$ by an involution $x \mapsto-x$ which is homeomorphic to a disk, and $\pi_{0}$ is a canonical projection $\pi_{0}:[x, t] \mapsto[x]$. In particular, $\partial \pi^{-1}(B)$ is homeomorphic to a Mobius strip $S^{1} \tilde{\times} \mathbb{R}$. On the other hand, $B^{\prime}:=S-\operatorname{int} B$ is homeomorphic to Mö. Then, the restriction $\pi: \pi^{-1}\left(B^{\prime}\right) \rightarrow B^{\prime}$ is a line bundle over Mö. In particular, it is trivial over $\partial B^{\prime}$. Namely, we have $\partial \pi^{-1}\left(B^{\prime}\right) \approx S^{1} \times \mathbb{R}$. This contradicts $\partial \pi^{-1}(B) \approx S^{1} \tilde{\times} \mathbb{R}$. Therefore, we obtain that if $S \approx P^{2}$, then $k=2$.

By a gluing argument as above, if $S \approx S^{2}$, then $k=2$ or 4 .

Explicitly, we determine the topology of $L(S ; k)$.

Corollary 2.56. $L\left(S^{2} ; 2\right)$ is isometric to $\hat{S}^{2} \times \mathbb{R} /(x, s) \sim(\sigma(x),-s)$, where $\hat{S}^{2}$ is a sphere of non-negative curvature in the sense of Alexandrov with an isometric involution $\sigma$ such that $\hat{S}^{2} / \sigma$ is isometric to the soul $S^{2}$ of $L\left(S^{2} ; 2\right)$.

$L\left(P^{2} ; 2\right)$ is isometric to $K^{2} \times \mathbb{R} /(x, s) \sim(\sigma(x),-s)$, where $K^{2}$ is a flat Klein bottle with an isometric involution $\sigma$ such that $K^{2} / \sigma$ is isometric to the soul $P^{2}$ of $L\left(P^{2} ; 2\right)$.

$L\left(S^{2} ; 4\right)$ is isometric to $T^{2} \times \mathbb{R} /(x, s) \sim(\sigma(x),-s)$, where $T^{2}$ is a flat torus with an isometric involution $\sigma$ such that $T^{2} / \sigma$ is isometric to the soul $S^{2}$ of $L\left(S^{2} ; 4\right)$.

Proof. To prove this, it suffices to determine the topology of a metric sphere around the soul of any $L(S ; k)$. For any $L(S ; k)$, we denote by $B(S ; k)$ a metric ball around 
$S$. Let us denote by $\pi$ a canonical projection

$$
\pi: B(S ; k) \rightarrow S
$$

Namely, for $x \in S, \pi(x)$ is the nearest point from $x$ in $S$.

We consider the case that $S \approx S^{2}$ and $k=2$. Let $p_{1}, p_{2} \in S$ be the topological singular points of $L\left(S^{2} ; 2\right)$ in $S$. We divide $S$ into $D_{1}$ and $D_{2}$ such that each $D_{i}$ is a disk neighborhood of $p_{i}$ and $D_{1} \cap D_{2}$ is homeomorphic to a circle. Then, for $i=1,2$, there is a homeomorphism $\varphi_{i}: \pi^{-1}\left(D_{i}\right) \rightarrow D^{2} \times[-1,1] /(x, s) \sim(-x,-s)$. The gluing part $\pi^{-1}\left(D_{1} \cap D_{2}\right)$ of $\pi^{-1}\left(D_{1}\right)$ and $\pi^{-1}\left(D_{2}\right)$ is homeomorphic to a Mobuis band Mö. Since the space $D^{2} \times[-1,1] / \sim$ is homeomorphic to $K_{1}\left(P^{2}\right)$, we obtain that $B\left(S^{2} ; 2\right)=\pi^{-1}\left(D_{1}\right) \cup \pi^{-1}\left(D_{2}\right)$ is homeomorphic to $K_{1}\left(P^{2}\right) \cup_{\text {Mö }} K_{1}\left(P^{2}\right)$ (see Remark 2.62 below). Then, $\partial B\left(S^{2} ; 2\right)$ is homeomorphic to a gluing of two copies of $P^{2}-\operatorname{int}(\mathrm{Mö}) \approx D^{2}$. Therefore, $\partial B\left(S^{2} ; 2\right) \approx S^{2}$.

We consider the case that $S \approx P^{2}$ and $k=2$. Let $p_{1}, p_{2} \in S$ be the topological singular points of $L\left(P^{2} ; 2\right)$ in $S$. We take a disk neighborhood $D$ of $\left\{p_{1}, p_{2}\right\}$ in $S$. Let us divide $D$ into $D_{1}$ and $D_{2}$ such that each $D_{i}$ is a disk neighborhood of $p_{i}$ and $D_{1} \cap D_{2}$ is homeomorphic to an interval. Then, $\pi^{-1}\left(D_{1} \cap D_{2}\right)$ is homeomorphic to $D^{2}$. Hence, $\pi^{-1}(D)=\pi^{-1}\left(D_{1}\right) \cup \pi^{-1}\left(D_{2}\right)$ is homeomorphic to $K_{1}\left(P^{2}\right) \cup_{D^{2}} K_{1}\left(P^{2}\right)$ (see Lemma 2.61). By Lemma 2.61, $\partial \pi^{-1}(D)$ is homeomorphic to a Klein bottle. Since $\pi$ is a non-trivial $I$-bundle over $\partial D_{i}$ for $i=1,2$, it is a trivial $I$-bundle over $\partial D$. Then, $\pi^{-1}(\partial D) \approx S^{1} \times I$. Let us set $A:=\partial B\left(P^{2} ; 2\right) \cap \pi^{-1}(D)$. Since $D$ has singular points $p_{1}$ and $p_{2}$ of the projection $\pi, A$ is connected, and hence $A$ is homeomorphic to $S^{1} \times I$.

Let us set $D^{\prime}:=S-\operatorname{int} D$ which is homeomorphic to Mö. Then, $\pi^{-1}\left(D^{\prime}\right)$ is homeomorphic to a total space of an $I$-bundle over Mö, which is Mö $\times I$ or Mö $\tilde{\times} I$. Let us set $A^{\prime}$ to be $\partial B\left(P^{2} ; 2\right) \cap \pi^{-1}\left(D^{\prime}\right)$. Therefore, if $\pi^{-1}\left(D^{\prime}\right) \approx$ Mö $\times I$, then $A^{\prime}$ is a disjoint union of two Mobius bands; and if $\pi^{-1}\left(D^{\prime}\right) \approx$ Mö $\tilde{\times} I$, then $A^{\prime}$ is homeomorphic to $S^{1} \times I$. Then, $\partial B\left(P^{2} ; 2\right)=A \cup A^{\prime}$ is homeomorphic to a Klein bottle if $\pi^{-1}\left(D^{\prime}\right) \approx \mathrm{Mo} \times I$ and is homeomorphic to $S^{1} \times I \cup_{\partial} S^{1} \times I$, which is a torus or a Klein bottle if $\pi^{-1}\left(D^{\prime}\right) \approx \operatorname{Mö} \tilde{\times} I$. Suppose that $\partial B\left(P^{2} ; 2\right)$ is homeomorphic to $T^{2}$. By Theorem 2.51, there is an involution on $T^{2}$ having only two fixed points. This is a contradiction (see [N, Lemma 3]). Therefore, $\partial B\left(P^{2} ; 2\right) \approx K^{2}$.

We consider the case that $S \approx S^{2}$ and $k=4$. Let $p_{1}, p_{2}, p_{3}, p_{4} \in S$ be all topological singular points of $L\left(S^{2} ; 4\right)$. Let $D$ and $D^{\prime}$ be domains in $S$ homeomorphic to a disk such that int $D$ (resp. int $D^{\prime}$ ) contains $p_{1}$ and $p_{2}$ (resp. $p_{3}$ and $p_{4}$ ), $D \cap D^{\prime}$ is homeomorphic to a circle and $S=D \cup D^{\prime}$. Let us denote $\partial B\left(S^{2} ; 4\right) \cap \pi^{-1}(D)$ (resp. $\left.\partial B\left(S^{2} ; 4\right) \cap \pi^{-1}\left(D^{\prime}\right)\right)$ by $A$ (resp. $\left.A^{\prime}\right)$. By repeating an argument similar to the case that $L(S ; k)=L\left(P^{2} ; 2\right)$, we obtain that $A$ and $A^{\prime}$ are homeomorphic to $S^{1} \times I$. Then, $\partial B\left(S^{2} ; 4\right)=A \cup A^{\prime}$ is homeomorphic to a torus or a Klein bottle. Suppose that $\partial B\left(S^{2} ; 4\right)$ is homeomorphic to $K^{2}$. By Theorem 2.51, there is an involution on $K^{2}$ having only four fixed points. This is a contradiction (see [N, Lemma 2]). Therefore, $\partial B\left(S^{2} ; 4\right) \approx T^{2}$.

Remark 2.57. Since involutions on closed surfaces are completely classified [N], the topology of each $L(S ; k)$ is unique.

For any space $L(S ; k)$, we denote a metric ball around $S$ in $L(S ; k)$ by $B(S ; k)$. The topology of any $B(S ; k)$ is as follows. 
Corollary 2.58. $B\left(S^{2} ; 2\right)$ is homeomorphic to $S^{2} \times[-1,1] / \mathbb{Z}_{2}, B\left(P^{2} ; 2\right)$ is homeomorphic to $K^{2} \times[-1,1] / \mathbb{Z}_{2}$, and $B\left(S^{2} ; 4\right)$ is homeomorphic to $T^{2} \times[-1,1] / \mathbb{Z}_{2}$. Here, all $\mathbb{Z}_{2}$-actions correspond to ones of Corollary 2.56 ,

Theorem 2.59 (Soul Theorem (Theorem 9.6 in [SY00])). Let $Y$ be a threedimensional open Alexandrov space and $S$ be its soul. Then we have the following:

(1) If $\operatorname{dim} S=0$, then $Y$ is homeomorphic to $\mathbb{R}^{3}$, or the cone $K\left(P^{2}\right)$ over the projective plane $P^{2}$, or $M_{\mathrm{pt}}$, which is defined in Example 1.2.

(2) If $\operatorname{dim} S=1$, then $Y$ is isometric to a quotient $(\mathbb{R} \times N) / \Lambda$, where $N$ is an Alexandrov space with non-negative curvature homeomorphic to $\mathbb{R}^{2}$ and $\Lambda$ is an infinite cyclic group. Here, the $\Lambda$-action is diagonal.

(3) If $\operatorname{dim} S=2$, then $Y$ is isometric to one of the normal bundle $N(S)=$ $L(S ; 0)$ over $S, L(S ; 2)$ and $L(S ; 4)$.

We will define examples of $L\left(S^{2} ; 2\right), L\left(P^{2} ; 2\right)$ and $L\left(S^{2} ; 4\right)$ in Example 2.63 .

Example 2.60 ([SY00, Example 9.3]). Let $\Gamma$ be a group of isometries generated by $\gamma$ and $\sigma$ on $\mathbb{R}^{3}$. Here, $\gamma$ and $\sigma$ are defined by $\gamma(x, y, z)=-(x, y, z)$ and $\sigma(x, y, z)=$ $(x+1, y, z)$. Then we obtain an open non-negatively curved Alexandrov space $\mathbb{R}^{3} / \Gamma$. This space is isometric to $M_{\mathrm{pt}}$ in Example 1.2.

We denote by $B(\mathrm{pt})$ a metric ball $B\left(p_{0}, R\right)$ around a soul $p_{0}$ of $M_{\mathrm{pt}}=\mathbb{R}^{3} / \Gamma$ for large $R>0$. Remark that $B(\mathrm{pt})$ is homeomorphic to $S^{1} \times D^{2} /(x, v) \sim(\bar{x},-v)$. We can check that $B(\mathrm{pt})$ is one of $K_{1}\left(P^{2}\right) \cup_{D^{2}} K_{1}\left(P^{2}\right)$. Here, $K_{1}\left(P^{2}\right) \cup_{D^{2}} K_{1}\left(P^{2}\right)$ denotes the gluing $K_{1}\left(P^{2}\right) \cup_{\varphi} K_{1}\left(P^{2}\right)$ of two copies $K_{1}\left(P^{2}\right)$ along domains $A_{1}$ and $A_{2}$ homeomorphic to $D^{2}$ contained in $\partial K_{1}\left(P^{2}\right) \approx P^{2}$ via a homeomorphism $\varphi: A_{1} \rightarrow A_{2}$. We show that the topology of $K_{1}\left(P^{2}\right) \cup_{D^{2}} K_{1}\left(P^{2}\right)$ does not depend on the choice of the gluing map.

Lemma 2.61. For any domains $A_{1}$ and $A_{2}$ which are homeomorphic to $D^{2}$ contained in $\partial K_{1}\left(P^{2}\right)$ and any homeomorphism $\varphi: A_{1} \rightarrow A_{2}$, there is a homeomorphism

$$
\tilde{\varphi}: K_{1}\left(P^{2}\right) \cup_{\varphi} K_{1}\left(P^{2}\right) \rightarrow K_{1}\left(P^{2}\right) \cup_{i d} K_{1}\left(P^{2}\right) .
$$

Here, id $: A_{0} \rightarrow A_{0}$ is the identity of a domain $A_{0}$ which is homeomorphic to $D^{2}$ contained in $\partial K_{1}\left(P^{2}\right)$. In particular, any such gluing is homeomorphic to $B(\mathrm{pt})$.

Proof. Let $X_{1}, X_{2}$ and $Y_{1}=Y_{2}$ be spaces homeomorphic to $K_{1}\left(P^{2}\right)$. Let us take domains $A_{1} \subset \partial X_{1}, A_{2} \subset \partial X_{2}$ and $A_{0} \subset \partial Y_{1}=\partial Y_{2}$ which are homeomorphic to $D^{2}$. Let us take any homeomorphism $\varphi: A_{1} \rightarrow A_{2}$.

Now let us fix a homeomorphism $\varphi_{1}: A_{1} \rightarrow A_{0}$. Then there is a homeomorphism $\hat{\varphi}_{1}: \partial X_{1} \rightarrow \partial Y_{1}$ which is an extension of $\varphi_{1}$. By using the cone structures of $X_{1}$ and $Y_{1}$, we obtain a homeomorphism $\tilde{\varphi}_{1}: X_{1} \rightarrow Y_{1}$ which is an extension of $\hat{\varphi}_{1}$. Let us set $\varphi_{2}:=\varphi_{1} \circ \varphi^{-1}: A_{2} \rightarrow A_{0}$. By an argument similar to the above, we obtain a homeomorphism $\tilde{\varphi}_{2}: X_{2} \rightarrow Y_{2}$ which is an extension of $\varphi_{2}$. We define a $\operatorname{map} \tilde{\varphi}: X_{1} \cup_{\varphi} X_{2} \rightarrow Y_{1} \cup_{i d_{A_{0}}} Y_{2}$ by

$$
\tilde{\varphi}(x)=\left\{\begin{array}{l}
\tilde{\varphi}_{1}(x) \text { if } x \in X_{1}, \\
\tilde{\varphi}_{2}(x) \text { if } x \in X_{2} .
\end{array}\right.
$$

This map is well-defined and a homeomorphism. 
Remark 2.62. We define a space $K_{1}\left(P^{2}\right) \cup_{\text {Mö }} K_{1}\left(P^{2}\right)$ in a way similar to $K_{1}\left(P^{2}\right) \cup_{D^{2}}$ $K_{1}\left(P^{2}\right)$. Let us consider domains $A_{1}, A_{2} \subset \partial K_{1}\left(P^{2}\right) \approx P^{2}$ which are homeomorphic to a Mobius band Mö, and take a homeomorphism $\varphi: A_{1} \rightarrow A_{2}$. Then, we denote $K_{1}\left(P^{2}\right) \cup_{\varphi} K_{1}\left(P^{2}\right)$ by the gluing $K_{1}\left(P^{2}\right) \cup_{\text {Mö }} K_{1}\left(P^{2}\right)$ for some gluing map $\varphi$. By an argument similar to the proof of Lemma 2.61, the topology of $K_{1}\left(P^{2}\right) \cup_{\text {Mö }} K_{1}\left(P^{2}\right)$ does not depend on the choice of the gluing map. We can show that any such gluing is homeomorphic to $S^{2} \times[-1,1] /(v, t) \sim(\sigma(v),-t)$. Here, $S^{2}$ is regarded as $\left\{v=(x, y, z) \in \mathbb{R}^{3}|| v \mid=1\right\}$ and $\sigma$ is an involution defined as $\sigma:(x, y, z) \mapsto(-x,-y, z)$. Further, it is homeomorphic to $B\left(P^{2} ; 2\right)$ (see Corollary 2.58).

$K_{1}\left(P^{2}\right) \cup_{\partial} K_{1}\left(P^{2}\right)$ denotes the gluing of two copies of $K_{1}\left(P^{2}\right)$ via a homeomorphism on $\partial K_{1}\left(P^{2}\right)$. This space has the same topology as $K_{1}\left(P^{2}\right) \cup_{i d} K_{1}\left(P^{2}\right)$, where $i d$ is the identity on $\partial K_{1}\left(P^{2}\right)$, which is homeomorphic to the suspension $\Sigma\left(P^{2}\right)$ over $P^{2}$. The proof is done by using the cone structure as in the proof of Lemma 2.61.

Example 2.63. We will define open Alexandrov spaces $L_{2}$ and $L_{4}$ as follows. Later, we show that $L_{k}$ is isometric to an $L(S ; k)$ for $k=2,4$.

Recall that $M_{\mathrm{pt}}$ is defined as

$$
M_{\mathrm{pt}}:=S^{1} \times \mathbb{R}^{2} /(x, y) \stackrel{\alpha}{\sim}(\bar{x},-y)
$$

in Example 1.2. We consider a closed domain $M_{\mathrm{pt}}^{\prime}$ of $M_{\mathrm{pt}}$ as

$$
M_{\mathrm{pt}}^{\prime}:=S^{1} \times[-\ell, \ell] \times \mathbb{R} / \alpha
$$

for some $\ell>0$. Then, $M_{\mathrm{pt}}^{\prime}$ is a convex subset of $M_{\mathrm{pt}}$, and hence it is an Alexandrov space of non-negative curvature with boundary $\partial M_{\mathrm{pt}}^{\prime} \equiv S^{1} \times \mathbb{R}$.

We denote by $L_{4}$ one of the open Alexandrov spaces of non-negative curvature defined as

$$
L_{4}(\varphi)=M_{\mathrm{pt}}^{\prime} \cup_{\varphi} M_{\mathrm{pt}}^{\prime}
$$

for an isometry $\varphi$ on $\partial M_{\mathrm{pt}}^{\prime}$. Here, we use the following notation: For Alexandrov spaces $A$ and $A^{\prime}$ whose boundaries are isometric to each other in the induced inner metric with an isometry $\varphi: \partial A \rightarrow \partial A^{\prime}, A \cup_{\varphi} A^{\prime}$ denotes the gluing of $A$ and $A^{\prime}$ via $\varphi$.

We will show that $L_{4}$ is $L\left(S^{2} ; 4\right)$ (Lemma 2.64).

Let $U_{2,1}$ be the Alexandrov space defined by

$$
U_{2,1}:=S^{1} \times \mathbb{R}^{2} /(x, y) \stackrel{\beta}{\sim}(-x,-y) .
$$

Let us set

$$
U_{2,1}^{\prime}:=S^{1} \times[-\ell, \ell] \times \mathbb{R} / \beta \subset U_{2,1}
$$

which is a convex subset of $U_{2,1}$, and hence it is an Alexandrov space of non-negative curvature with boundary $\partial U_{2,1}^{\prime} \equiv S^{1} \times \mathbb{R}$. Let us set $S\left(U_{2,1}^{\prime}\right):=S^{1} \times[-\ell, \ell] \times\{0\} / \beta$. Note that $S\left(U_{2,1}^{\prime}\right)$ is isometric to a Mobius band Mö and $U_{2,1}^{\prime}$ is isomorphic to an $\mathbb{R}$-bundle over $S\left(U_{2,1}^{\prime}\right)$.

We define open Alexandrov spaces $L_{2,1}, L_{2,2}$ and $L_{2,3}$ of non-negative curvature as

$$
\begin{aligned}
& L_{2,1}:=L_{2,1}(\varphi)=M_{\mathrm{pt}}^{\prime} \cup_{\varphi} U_{2,1}^{\prime}, \\
& L_{2,2}:=L_{2,2}(\varphi)=M_{\mathrm{pt}}^{\prime} \cup_{\varphi} D^{2} \times \mathbb{R}, \text { and } \\
& L_{2,3}:=L_{2,3}(\varphi)=M_{\mathrm{pt}}^{\prime} \cup_{\varphi} \mathrm{Mö} \times \mathbb{R} .
\end{aligned}
$$


Here, $\varphi$ denotes a gluing isometry between the corresponding boundaries, $D^{2}$ denotes a two-disk of non-negative curvature, and Mö is a flat Mobius band.

Let us define an Alexandrov space $A$ of non-negative curvature

$$
A:=[-a, a] \times[-b, b] \times \mathbb{R} /(v, s) \sim(-v,-s) .
$$

Here, $v \in[-a, a] \times[-b, b]$ and $s \in \mathbb{R}$. The boundary $\partial A$ is isometric to $S^{1} \times \mathbb{R}$. We define an open Alexandrov space $L_{2,4}$ of non-negative curvature as

$$
L_{2,4}=L_{2,4}(\varphi)=A \cup_{\varphi} A
$$

for some isometry $\varphi$ on $\partial A$.

We will prove that $L_{2,1}$ and $L_{2,3}$ are $L\left(P^{2} ; 2\right)$ and $L_{2,2}$ and $L_{2,4}$ are $L\left(S^{2} ; 2\right)$ (Lemma 2.65).

From now on throughout this paper, we denote by $L_{2}$ one of $L_{2,1}, L_{2,2}, L_{2,3}$ and $L_{2,4}$.

Lemma 2.64. $L_{4}$ is $L\left(S^{2} ; 4\right)$.

Proof. Recall that $L_{4}=L_{4}(\varphi)=M_{\mathrm{pt}}^{\prime} \cup_{\varphi} M_{\mathrm{pt}}^{\prime}$. We identify $\partial M_{\mathrm{pt}}^{\prime}$ as $S^{1} \times \mathbb{R}$ via an isometry $[\xi, \ell, s] \mapsto[\xi, s]$. The isometry $\varphi: \partial M_{\mathrm{pt}}^{\prime} \rightarrow \partial M_{\mathrm{pt}}^{\prime}$ is written as

$$
\varphi[\xi, \ell, s]=[f(\xi), \ell, g(s)]
$$

for some isometries $f$ on $S^{1}$ and $g$ on $\mathbb{R}$. Then, $g(s)=( \pm 1) \cdot s+g(0)$.

Let us define $E:=[-\ell, \ell] \times \mathbb{R} /(s, t) \sim(-s,-t)$. Obviously, there is a canonical projection $\pi: M_{\mathrm{pt}}^{\prime} \rightarrow E$ defined by $[\xi, s, t] \mapsto[s, t]$. Here, $\xi \in S^{1}, s \in[-\ell, \ell]$ and $t \in \mathbb{R}$. The map $\pi$ is a line bundle over $E-\{[0,0]\}$.

For $a \in \mathbb{R}$, let us define $S_{\mathrm{pt}}^{\prime}(a) \subset M_{\mathrm{pt}}^{\prime}$ as

$$
S_{\mathrm{pt}}^{\prime}(a):=S^{1} \times\{(t, a t / \ell) \mid t \in[-\ell, \ell]\} / \alpha .
$$

$S_{\mathrm{pt}}^{\prime}(a)$ is homeomorphic to a disk. Then, by using the fibration $\pi: M_{\mathrm{pt}}^{\prime} \rightarrow E$, we obtain that $M_{\mathrm{pt}}^{\prime}$ is homotopic to $S_{\mathrm{pt}}^{\prime}(a)$ for any $a \in \mathbb{R}$.

By choosing $a$ with respect to $g(0)$, we obtain that $L_{4}$ is homotopic to the gluing $S_{\mathrm{pt}}^{\prime}(a) \cup_{\partial} S_{\mathrm{pt}}^{\prime}(-a)$ which is homeomorphic to $S^{2}$. Thus, a soul of $L_{4}$ is homeomorphic to a sphere. Since $M_{\mathrm{pt}}^{\prime}$ has only two topological singular points in its interior, $L_{4}$ has only four topological singular points. Therefore, $L_{4}$ is $L\left(S^{2} ; 4\right)$.

Lemma 2.65. $L_{2,1}$ and $L_{2,3}$ are $L\left(P^{2} ; 2\right)$, and $L_{2,2}$ and $L_{2,4}$ are $L\left(S^{2} ; 2\right)$.

Proof. We will use the same notation as in the proof of Lemma 2.64

Let us consider $L_{2,1}=L_{2,1}(\varphi)=M_{\mathrm{pt}}^{\prime} \cup_{\varphi} U_{2,1}$. Recall that $U_{2,1}^{\prime} \subset U_{2,1}$ is isomorphic to a line bundle over $S\left(U_{2,1}^{\prime}\right)$, where $S\left(U_{2,1}^{\prime}\right)$ is a subset of $U_{2,1}^{\prime}$ homeomorphic to Mö. By using the bundle structure of $U_{2,1}^{\prime}$ and the fibration $\pi$, we obtain that $L_{2,1}$ is homotopic to the gluing $S_{\mathrm{pt}}^{\prime} \cup_{\partial} S\left(U_{2,1}^{\prime}\right)$, which is homeomorphic to $P^{2}$. Since $L_{2,1}$ has only two topological singular points, it follows that $L_{2,1}$ is $L\left(P^{2} ; 1\right)$.

Let us take $L_{2,2}=L_{2,2}(\varphi)=M_{\mathrm{pt}}^{\prime} \cup_{\varphi} D^{2} \times \mathbb{R}$. By using the fibration $\pi$, we obtain that $L_{2,2}$ is homotopic to the gluing $S_{\mathrm{pt}}^{\prime}(a) \cup_{\partial} D^{2}$ for some $a$, which is homeomorphic to $S^{2}$. Also, $L_{2,2}$ has only two topological singular points. This implies that $L_{2,2}$ is $L\left(S^{2} ; 2\right)$.

Let us take $L_{2,3}=L_{2,3}(\varphi)=M_{\mathrm{pt}}^{\prime} \cup_{\varphi} \mathrm{Mö} \times \mathbb{R}$. By using $\pi$, we obtain that $L_{2,3}$ is homotopic to the gluing $S_{\mathrm{pt}}^{\prime}(a) \cup \cup_{\partial}$ Mö for some $a$, which is homeomorphic to $P^{2}$. Since $L_{2,3}$ has only two topological singular points, it follows that $L_{2,3}$ is $L\left(P^{2} ; 2\right)$. 
Let us take $L_{2,4}=L_{2,4}(\varphi)=A \cup_{\varphi} A$. Recall that $A=[-a, a] \times[-b, b] \times$ $\mathbb{R} /(x, y, s) \sim(-x,-y,-s)$. Let us consider a subset $S^{\prime}:=[-a, a] \times[-b, b] \times\{0\} / \sim$ of $A$, which is homeomorphic to a disk. Let us set $E:=[-b, b] \times \mathbb{R} /(y, s) \sim(-y,-s)$. There is a canonical projection $\pi^{\prime}: A \rightarrow E$ defined by $\pi^{\prime}([x, y, s])=[y, s]$. By using it, we obtain that $L_{2,4}$ is homotopic to $S^{\prime} \cup_{\partial} S^{\prime}$, which is homeomorphic to $S^{2}$. Since $L_{2,4}$ has only two topological singular points, it follows that $L_{2,4}$ is $L\left(S^{2} ; 2\right)$.

2.9. Classification of Alexandrov surfaces from SY00. We recall a result for a classification of Alexandrov surfaces by quoting SY00.

Proposition 2.66 (The Gauss-Bonnet Theorem [SY00, Proposition 14.1]). If X is a compact Alexandrov surface, then we have

$$
\omega(X)+\kappa(\partial X)=2 \pi \chi(X) .
$$

Proposition 2.67 (The Cohn-Vossen Theorem [SY00, Proposition 14.2]). If $X$ is a non-compact Alexandrov surface, then we have

$$
2 \pi \chi(X)-\pi \chi(\partial X)-\omega(X)-\kappa(\partial X) \geq 0 .
$$

Theorem 2.68 ([SY00, Corollary 14.4]). Let $X$ be a non-negatively curved Alexandrov surface. Then, the following hold:

(1) $X$ is homeomorphic to either $\mathbb{R}^{2}, \mathbb{R}_{>0} \times \mathbb{R}, S^{2}, P^{2}, D^{2}$ or isometric to $[0, \ell] \times \mathbb{R},[0, \ell] \times S^{1}(r), \mathbb{R}_{\geq 0} \times S^{1}(r), \mathbb{R} \times S^{1}(r), \mathbb{R} \times S^{1}(r) / \mathbb{Z}_{2}$, a flat torus, or a flat Klein bottle for some $\ell, r>0$.

(2) int $X$ contains at most four essential singular points, and denoting by $n$ the number of essential singular points in int $X$, we have the following for some $\ell, r>0$.

(a) If $n \geq 1, X$ is either homeomorphic to $\mathbb{R}^{2}, S^{2}, P^{2}, D^{2}$ or isometric to $\operatorname{dbl}\left(\mathbb{R}_{\geq 0} \times \mathbb{R}_{\geq 0}\right) \cap\{(x, y) \mid y \leq h\}$.

(b) If $n \geq 2, X$ is either homeomorphic to $S^{2}$ or isometric to $\mathrm{dbl}\left(\mathbb{R}_{\geq 0} \times\right.$ $[0, h]), \operatorname{dbl}\left(\mathbb{R}_{\geq 0} \times[0, h]\right) \cap\{(x, y) \mid x \leq \ell\}$ or $\mathrm{dbl}([0, \ell] \times[0, h]) / \mathbb{Z}_{2}$.

(c) If $n \geq 3$, then $X$ is homeomorphic to $S^{2}$.

(d) If $n=4, X$ is isometric to $A \cup_{\phi} B$, where $A$ and $B$ are isometric to $\operatorname{dbl}([0, \ell] \times[0, \infty)) \cap\{(x, y) \mid y \leq a\}$ and $\operatorname{dbl}([0, \ell] \times[0, \infty)) \cap\{(x, y) \mid y \leq$ $b\}$ for some $a, b>0$, respectively, and $\phi: \partial A \rightarrow \partial B$ is some isometry.

2.10. A fundamental observation. In this subsection, we prove fundamental propositions on the sets of topologically singular points of Alexandrov spaces.

First, we note the following proposition on the number of topologically singular points of a three-dimensional closed Alexandrov space. Let us consider a $(2 n+1)$ dimensional manifold $X$ such that its boundary $\partial X$ is homeomorphic to the disjoint union $\bigsqcup_{i=1}^{m} P^{2 n}$ of the projective spaces. Then we see that $m$ is even. Indeed, we consider the double $\operatorname{dbl}(X)$ and its Euler number:

$$
0=\chi(\operatorname{dbl}(X))=2 \chi(X)-\chi(\partial X)=2 \chi(X)-m .
$$

Proposition 2.69. Let $M$ be a three-dimensional closed Alexandrov space. Then the number of topologically singular points of $M$ is even.

Proof. Since $M$ is compact, $S_{\text {top }}(M)$ is a finite set. By Theorem 2.34, there exists $r>0$ such that for any $p \in S_{\text {top }}(M)$ we have $(B(p, r), p) \approx\left(K_{1}\left(P^{2}\right), o\right)$. Therefore,

$$
M_{0}:=M-\bigcup_{p \in S_{\text {top }}(M)} U(p, r)
$$


is a manifold with boundary $\partial M_{0} \approx \bigsqcup_{p \in S_{\text {top }}(M)} P^{2}$. By the above argument, $\sharp S_{\text {top }}(M)$ is even.

We also prepare the following proposition.

Proposition 2.70. Let $\left(M_{i}, p_{i}\right)$ be a sequence of $n$-dimensional pointed Alexandrov spaces of curvature $\geq-1$ converging to $(X, p)$. If diam $\Sigma_{p}>\pi / 2$, then $\Sigma_{p_{i}}$ is homeomorphic to a suspension over an Alexandrov space of curvature $\geq 1$, for large $i$.

Proof. Suppose that the conclusion fails. Then we have some sequence $\left\{M_{i}^{n}\right\}$ such that $\left(M_{i}, p_{i}\right)$ converges to $(X, p)$ and each $\Sigma_{p_{i}}$ does not have topological suspension structure over any Alexandrov space of curvature $\geq 1$. It follows from Theorem 2.35 that diam $\left(\Sigma_{p_{i}}\right) \leq \pi / 2$. The convergence of spaces of directions is lower semicontinuous:

$$
\liminf _{i \rightarrow \infty} \Sigma_{p_{i}} \geq \Sigma_{p}
$$

Then we have $\operatorname{diam}\left(\Sigma_{p}\right) \leq \pi / 2$. This is a contradiction.

\section{Smooth Approximations And Flow ARguments}

\subsection{Flow Theorem.}

A bijective map $f: X \rightarrow Y$ between metric spaces is called bi-Lipschitz if both $f$ and $f^{-1}$ are Lipschitz.

Definition 3.1. Let $M$ be a topological space. A continuous map $\Phi: M \times \mathbb{R} \rightarrow M$ is called a flow if it satisfies

$$
\begin{aligned}
\Phi(x, 0) & =x, \\
\Phi(x, s+t) & =\Phi(\Phi(x, s), t)
\end{aligned}
$$

for any $x \in M$ and $s, t \in \mathbb{R}$. Remark that, for each $t \in \mathbb{R}$, the map

$$
\Phi_{t}=\Phi(\cdot, t): M \rightarrow M
$$

has the inverse map $\Phi_{-t}$.

Let $M$ be a metric space. If a flow $\Phi$ is a Lipschitz map from $M \times \mathbb{R}$ to $M$, then we call it a Lipschitz flow. Remark that for any Lipschitz flow $\Phi, \Phi(\cdot, t)$ is bi-Lipschitz for each $t \in \mathbb{R}$.

By using the proof of Theorem 2.46, we obtain the following theorem. This is a main tool for the proof of our results throughout the present paper.

Theorem 3.2 (Flow Theorem). For any $n \in \mathbb{N}$, there exists a positive number $\varepsilon_{n}$ depending only on $n$ satisfying the following: Let $C$ be a compact subset and $S$ be a closed subset in an $n$-dimensional Alexandrov space $M$ with curvature $\geq-1$. Suppose that $C \cap S=\emptyset$ and $C$ is $\varepsilon$-strained and dist $_{S}$ is $(1-\delta)$-regular on $C$ for $\delta>0$, where $0<\varepsilon \leq \varepsilon_{n}$ and $\delta$ is smaller than some constant. Then there exist a neighborhood $U(C)$ of $C$ and a Lipschitz flow $\Phi: M \times \mathbb{R} \rightarrow M$ satisfying the following:

(i) For any $x \in U(C)$, putting $I_{x}:=\{t \in \mathbb{R} \mid \Phi(x, t) \in U(C)\}, \Phi(x, t)$ is a $5 \sqrt{\delta}+\theta(\varepsilon)$-isometric embedding in $t \in I_{x}$. 
(ii) The speed with which $\Phi$ leaves $S$ is almost one. Namely,

$$
\left.\frac{d}{d t}\right|_{t=0+} \operatorname{dist}_{S} \circ \Phi(x, t)>1-5 \sqrt{\delta}-\theta(\varepsilon)
$$

at any $x \in U(C)$.

Proof of Theorem 3.2. To prove this, we must remember the proof of Theorem 2.46 in reference to $\mathrm{KMS}$, and $\mathrm{O}$.

For a while, $x$ denotes an arbitrary point in $C$. We set

$$
v(x):=\frac{\nabla \operatorname{dist}_{S}}{\left|\nabla \operatorname{dist}_{S}\right|} \in \Sigma_{x} M .
$$

Since $d_{S}$ is $(1-\delta)$-regular, we have

$$
\left(\operatorname{dist}_{S}\right)_{x}^{\prime}(v(x))=-\cos \angle\left(S_{x}^{\prime}, v(x)\right)>1-\delta .
$$

We fix a point $q(x) \in S$ such that

$$
|x q(x)|=|x S| .
$$

Then, by (3.2), we have

$$
\angle\left(q(x)^{\prime}, v(x)\right) \geq \angle\left(S^{\prime}, v(x)\right)>\pi-\delta^{\prime} .
$$

Here, $\delta^{\prime}:=\pi-\cos ^{-1}(-1+\delta)$. Note that $\lim _{\delta \rightarrow 0} \frac{\delta^{\prime}}{\sqrt{\delta}}=\frac{1}{2 \sqrt{2}}$.

We put $\ell:=\min \{\varepsilon$-str. $\operatorname{rad}(C), d(S, C)\}$. We fix positive numbers $s$ and $t$ with $s \ll t \ll \ell$. Take a maximal $0.2 s$-net $\left\{x_{j} \mid j=1, \ldots, N\right\}$ of $C$. Fix any $j \in$ $\{1, \ldots, N\}$. We take $\varepsilon$-strainer $\left\{q_{j}^{\alpha} \mid \alpha= \pm 1, \ldots, \pm n\right\}$ at $x_{j}$ of length $\geq \ell$. We may assume that $\left\{q_{j}^{\alpha}\right\}$ satisfies the following:

$$
q_{j}^{+1}=q\left(x_{j}\right)
$$

Since $t \ll \ell,\left\{q_{j}^{\alpha}\right\}$ is also $\theta(\varepsilon)$-strainer at any $x \in B\left(x_{j}, 10 t\right)$. It follows from $s \ll t$ and [Y conv, Lemma 1.9] that

$$
\left|\tilde{\angle} q_{j}^{\alpha} x y-\angle q_{j}^{\alpha} x y\right|<\theta(\varepsilon)
$$

for any $x \in B\left(x_{j}, s\right)$ and $y \in B(x, s)$.

We denote by $E_{j}$ the standard $n$-dimensional Euclidean space. Define a map

$$
f_{j}=\left(f_{j}^{\alpha}\right)_{\alpha=1}^{n}: B\left(x_{j}, 10 t\right) \rightarrow E_{j}
$$

by

$$
f_{j}^{\alpha}(y)=\frac{1}{\mathcal{H}^{n}\left(B\left(q_{j}^{\alpha}, \varepsilon^{\prime}\right)\right)} \int_{z \in B\left(q_{j}^{\alpha}, \varepsilon^{\prime}\right)} d(y, z)-d\left(x_{j}, z\right) d \mathcal{H}^{n}(z)
$$

where $\varepsilon^{\prime} \ll \varepsilon$. This map is a $\theta(\varepsilon)$-almost isometric $D C^{1}$-homeomorphism, which is actually a $D C^{1}$-coordinate system.

Lemma $3.3\left(\left[\mathrm{O}\right.\right.$, Lemma 5]). There is an isometry $F_{j}^{k}: E_{k} \rightarrow E_{j}$ satisfying the following:

$$
\begin{aligned}
\left|F_{j}^{k} \circ f_{k}(y)-f_{j}(y)\right| & <\theta(\varepsilon) s, \\
\left|d F_{j}^{k} \circ d f_{k}(\xi)-d f_{j}(\xi)\right| & <\theta(\varepsilon)
\end{aligned}
$$

for any $j$ and $k$, and $y \in B\left(x_{j}, s\right) \cap B\left(x_{k}, s\right)$ and $\xi \in \Sigma_{y}$.

Remark that each $f_{j}$ has the directional derivative $d f_{j}$. 
Proof of Lemma 3.3. We first recall how to define $F_{j}^{k}$ 's. The property (3.6) is proved in the same way to the original proof of [O, Lemma 5] in our situation. We only prove (3.7).

Fix any $j$ and $k$. For $\alpha=1, \ldots, n$, take $y_{k}^{\alpha} \in x_{k} q_{k}^{-\alpha}$ and $y_{k}^{-\alpha} \in x_{k} q_{k}^{\alpha}$ such that

$$
\left|x_{k} y_{k}^{\alpha}\right|=\left|x_{k} y_{k}^{-\alpha}\right|=s \text {. }
$$

Then we have

$$
\left\langle f_{k}\left(y_{k}^{\alpha}\right), f_{k}\left(y_{k}^{\beta}\right)\right\rangle=s^{2} \delta_{\alpha \beta}+\theta(\varepsilon, s / \ell)
$$

for all $\alpha, \beta=1, \ldots, n$. Here $\langle\cdot, \cdot\rangle$ is the standard inner product on $E_{k}$. Since $s \ll \ell$, $\theta(\varepsilon, s / \ell)=\theta(\varepsilon)$. Then, we have

$$
\left|f_{k}\left(y_{k}^{\alpha}\right)-s e_{k}^{\alpha}\right|<\theta(\varepsilon) .
$$

Here, $\left\{e_{k}^{\alpha}\right\}_{\alpha=1}^{n}$ is an o.n.b on $E_{k}$. In a similar way, we have

$$
\left|f_{k}\left(y_{k}^{-\alpha}\right)+s e_{k}^{\alpha}\right|<\theta(\varepsilon) .
$$

We define vectors $v^{\alpha}, w^{\alpha} \in E_{j}(\alpha=1, \ldots, n)$ by

$$
v^{\alpha}:=\frac{1}{2 s}\left\{f_{j}\left(y_{k}^{\alpha}\right)-f_{j}\left(y_{k}^{-\alpha}\right)\right\}
$$

Then, we have

$$
\left\langle v^{\alpha}, v^{\beta}\right\rangle=\delta_{\alpha \beta}+\theta(\varepsilon) .
$$

Then, $\left\{v_{\alpha}\right\}$ is an almost orthonormal basis. By Schmidt's orthogonalization we obtain an orthonormal basis $\left\{\tilde{e}_{\alpha}\right\}$ of $E_{j}$ such that

$$
\left|\tilde{e}_{\alpha}-v_{\alpha}\right|<\theta(\varepsilon) \text {. }
$$

We now define an isometry $F_{j}^{k}: E_{k} \rightarrow E_{j}$ by changing the orthonormal basis and the translation:

$$
F_{j}^{k}(v)=f_{j}\left(x_{k}\right)+\sum_{\alpha=1}^{n}\left\langle v, e_{k}^{\alpha}\right\rangle \tilde{e}_{\alpha} .
$$

Then, we have

$$
F_{j}^{k}\left(f_{k}(x)\right)=f_{j}(x)+s \vec{v}(\theta(\varepsilon))
$$

for all $x \in B\left(x_{j}, s\right)$. Here, $\vec{v}(c)$ is a vector whose norm is less than or equal to $|c|$.

We prove (3.7). For any $y \in B\left(x_{j}, s\right) \cap B\left(x_{k}, s\right)$ and $\xi \in \Sigma_{y}$, by Lemma 2.17. there exists $z \in M$ such that

$$
|y z|=t \text { and } \angle(\xi, \uparrow \underset{y}{z})=\theta(\varepsilon) .
$$

Then, we have

$$
\tilde{\angle} q_{j}^{\alpha} y z=\angle\left(\left(q_{j}^{\alpha}\right)^{\prime}, z^{\prime}\right)+\theta(s / t) .
$$

Since $s \ll t$, we have $\theta(s / t)=\theta(\varepsilon)$. Therefore,

$$
\begin{aligned}
d_{y} f_{j}^{\alpha}(\xi) & =\frac{1}{\mathcal{H}^{n}\left(B\left(q_{j}^{\alpha}, \varepsilon^{\prime}\right)\right)} \int_{w \in B\left(q_{j}^{\alpha}, \varepsilon^{\prime}\right)}-\cos \angle\left(w_{y}^{\prime}, \xi\right) d \mathcal{H}^{n}(w) \\
& =-\cos \tilde{\angle} q_{j}^{\alpha} y z+\theta(\varepsilon) .
\end{aligned}
$$


On the other hand,

$$
\begin{aligned}
d F_{j}^{k} \circ d f_{k}(\xi) & =d F_{j}^{k}\left(\left(-\cos \tilde{\angle} q_{j}^{\alpha} y z\right)_{\alpha=1, \ldots, n}\right)+\vec{v}(\theta(\varepsilon)) \\
& =\sum_{\alpha}-\cos \tilde{\angle} q_{j}^{\alpha} y z \cdot \tilde{e}_{\alpha}+\vec{v}(\theta(\varepsilon)) .
\end{aligned}
$$

Therefore, we have (3.7).

Set $V_{j}:=B(0,0.4 s) \subset E_{j}$ for all $j$.

Next, we perturb $\left\{F_{j}^{k}\right\}$ to a family $\left\{\tilde{F}_{j}^{k}\right\}$ satisfying the following.

Lemma 3.4 ([0, Lemma 6]). For any $j$ and $k$ with $d\left(x_{j}, x_{k}\right)<0.9 s$, there exists a $\theta(\varepsilon)$-almost isometric $C^{\infty}$ map $\tilde{F}_{j}^{k}: V_{k} \rightarrow E_{j}$ satisfying the following:

$$
\begin{aligned}
\tilde{F}_{j}^{j} & =\mathrm{id} \text { on } E_{j} \text { and } \\
\tilde{F}_{j}^{l}(v) & =\tilde{F}_{j}^{k} \circ \tilde{F}_{k}^{l}(v)
\end{aligned}
$$

for any $j$ and $k$ with $d\left(x_{j}, x_{k}\right)<0.9 s$ and $v \in V_{l} \cap \tilde{F}_{l}^{k}\left(V_{k}\right) \cap \tilde{F}_{l}^{j}\left(V_{j}\right)$.

Moreover, we can obtain this perturbed $\left\{\tilde{F}_{j}^{k}\right\}$ also satisfying (3.6) and (3.7). That is, we have

$$
\begin{aligned}
\left|\tilde{F}_{j}^{k} \circ f_{k}(y)-f_{j}(y)\right| & <\theta(\varepsilon) s, \\
\left|d \tilde{F}_{j}^{k} \circ d f_{k}(\xi)-d f_{j}(\xi)\right| & <\theta(\varepsilon)
\end{aligned}
$$

for any $j$ and $k$, and $y \in B\left(x_{j}, s\right) \cap B\left(x_{k}, s\right)$ and $\xi \in \Sigma_{y}$.

Proof. We only review the first step of construction of $\tilde{F}_{j}^{k}$ 's by induction referring to the proof of [O].

Let us first review how to construct $\tilde{F}_{j}^{k}$ 's. Let $\phi:[0, \infty) \rightarrow[0, \infty)$ be a $C^{\infty}$ function such that

$$
\begin{aligned}
\phi & =1 \text { on }[0,1 / 2], \\
\phi & =0 \text { on }[1, \infty), \text { and } \\
-4 & \leq \phi^{\prime} \leq 0 .
\end{aligned}
$$

Set

for $v \in V_{j}$.

$$
\psi_{j}(v):=\phi(|v| / 0.8 s)
$$

We set $\tilde{F}_{j}^{1}=F_{j}^{1}$ and $\tilde{F}_{1}^{j}=\left(\tilde{F}_{j}^{1}\right)^{-1}$, and define $\tilde{F}_{j}^{2}: U_{2} \rightarrow \mathbb{R}^{n}$ for $j \geq 2$ by

$$
\tilde{F}_{j}^{2}(v):=\psi_{1} \circ \tilde{F}_{1}^{2}(v) \cdot \tilde{F}_{j}^{1} \circ \tilde{F}_{1}^{2}(v)+\left(1-\psi_{1} \circ \tilde{F}_{1}^{2}(v)\right) \cdot F_{j}^{2}(v)
$$

for $v \in V_{2}$. By construction, $\tilde{F}_{j}^{2}$ is smooth and satisfies (3.15) and (3.16).

For $v \in V_{2}$, we have

$$
\begin{aligned}
\phi_{1} \circ \tilde{F}_{1}^{2}(v) & =1+\theta(\varepsilon)|v|, \\
\left\|d\left(\phi_{1} \circ \tilde{F}_{1}^{2}\right)\right\|_{C^{1}} & =\theta(\varepsilon) .
\end{aligned}
$$

Therefore, we have, for any $v \in V_{2}$ and $w \in T_{v} E_{2}$ with $|w|=1$,

$$
\begin{aligned}
d \tilde{F}_{j}^{2}(w) & =d\left(\tilde{F}_{j}^{1} \circ \tilde{F}_{1}^{2}\right)(w)+\theta(\varepsilon) \\
& =d\left(F_{j}^{1} \circ F_{1}^{2}\right)(w)+\theta(\varepsilon) \\
& =d F_{j}^{2}(w)+\theta(\varepsilon) .
\end{aligned}
$$


Thus, we have

$$
\left\|d \tilde{F}_{j}^{2}-d F_{j}^{2}\right\|<\theta(\varepsilon)
$$

at any $v \in V_{2}$.

Therefore, for a segment $c:\left[0, t_{0}\right] \rightarrow V_{2}$ between $v$ and $y$, we have

$$
\left|\tilde{F}_{j}^{2}(v)-\tilde{F}_{j}^{2}(w)\right|=\left|\int_{0}^{t_{0}} d \tilde{F}_{j}^{2}\left(c^{\prime}(t)\right) d t\right| \geq 0.9|v-w| .
$$

Thus, $\tilde{F}_{j}^{2}$ is injective.

By the chain rule (3.13), an equivalence relation $\sim$ on the disjoint union $\bigsqcup_{j} V_{j}$ is defined in the following natural way: $V_{j} \ni y \sim y^{\prime} \in V_{k} \Longleftrightarrow \tilde{F}_{j}^{k}\left(y^{\prime}\right)=y$. Set $N:=\bigsqcup V_{j} / \sim$. We denote by $\pi$ the projection

$$
\pi: \bigsqcup_{j=1}^{N} V_{j} \rightarrow N
$$

We denote by $\tilde{V}_{j}:=\pi\left(V_{j}\right)$ the subset of $N$ corresponding to $V_{j}$, and by $\pi_{j}$ the restriction of $\pi$

$$
\pi_{j}: V_{j} \rightarrow \tilde{V}_{j}
$$

We define $\tilde{f}_{j}:=\pi_{j}^{-1}$. Then $N$ is a $C^{\infty}$-manifold with atlas $\left\{\left(\tilde{V}_{j}, \tilde{f}_{j}\right)\right\}_{j}$, and $\tilde{F}_{j}^{k}$ : $\tilde{f}_{k}\left(\tilde{V}_{k} \cap \tilde{V}_{j}\right) \rightarrow \tilde{f}_{j}\left(\tilde{V}_{k} \cap \tilde{V}_{j}\right)$ is the associate transformation.

Define maps $f^{(j)}: B\left(x_{j}, s\right) \rightarrow E_{j}(j=1, \ldots, N)$ by

$$
\begin{aligned}
& f^{(1)}(x):=f_{1}(x), \\
& f^{(2)}(x):=\psi_{1} \circ f^{(1)}(x) \cdot \tilde{F}_{2}^{1} \circ f^{(1)}(x)+\left(1-\psi_{1} \circ f^{(1)}(x)\right) f_{2}(x),
\end{aligned}
$$

Set $\hat{V}_{j}:=f^{(j)-1}\left(V_{j}\right)$. Then we have

$$
f^{(j)}=\tilde{F}_{j}^{k} \circ f^{(k)}
$$

on $\hat{V}_{j} \cap \hat{V}_{k}$. Indeed, for instance, $f^{(2)}=\tilde{F}_{2}^{1} \circ f^{(1)}$ on $B_{1} \cap B_{2}$. For the general case, we refer to [O, pp. 1272-1273]. Set $U:=\bigcup_{j} \hat{V}_{j}$. A homeomorphism $f: U \rightarrow N$ is defined to be the inductive limit of $\pi \circ f^{(j)}$.

By [O, Lemma 8], we obtain the following properties of $f^{(j)}$ :

$$
\begin{gathered}
\left|f_{j}(x)-f^{(j)}(x)\right|<\theta(\varepsilon) s, \\
\left|d f_{j}(\xi)-d f^{(j)}(\xi)\right|<\theta(\varepsilon)
\end{gathered}
$$

for all $x \in B\left(x_{j}, 0.4 s\right)$ and $\xi \in \Sigma_{x}$.

Let $\left\{\chi_{j}\right\}_{j}$ be a smooth partition of unity such that $\operatorname{supp}\left(\chi_{j}\right) \subset \tilde{V}_{j}$. The desired Riemannian metric $g_{N}$ on $N$ is defined by

$$
\left(g_{N}\right)_{x}(v, w):=\sum_{j} \chi_{j}(x)\left\langle d \tilde{f}_{j}(v), d \tilde{f}_{j}(w)\right\rangle
$$

for any $x \in N$ and $v, w \in T_{x} N$.

Up to here, we reviewed the construction of a smooth approximation $f: U \rightarrow N$ by [KMS (and [0]). Next, we construct the desired flow. 
We first remark that

Lemma 3.5. For each $j, f^{(j)-1}: V_{j} \rightarrow \hat{V}_{j}$ is differentiable. Hence, $f$ and $f^{-1}$ are also differentiable.

Proof. Since $f^{(j)}$ is differentiable, for any scale $(o)$, the following diagram commutes:

$$
\begin{array}{ccc}
\left(\hat{V}_{j}\right)_{x}^{(o)} \stackrel{\left(f^{(j)}\right)_{x}^{(o)}}{\longrightarrow} & \left(V_{j}\right)_{y}^{(o)} \\
\hat{\rho}^{(o)} \downarrow & & \downarrow \rho^{(o)} \\
T_{x} M & \underset{d_{x} f^{(j)}}{\longrightarrow} & T_{y} E_{j}
\end{array}
$$

where $y:=f^{(j)}(x)$ and $\hat{\rho}^{(o)}$ and $\rho^{(o)}$ are canonical isometries. We will omit the symbol $(o)$ to write $\hat{\rho}:=\hat{\rho}^{(o)}$ and $\rho:=\rho^{(o)}$.

Since $f^{(j)}$ is $\theta(\varepsilon)$-isometric, $\left(f^{(j)}\right)_{x}^{(o)}$ and $\left(f^{(j)-1}\right)_{y}^{(o)}$ are also. We define a map $A: T_{y} E_{j} \rightarrow T_{x} M$ by

$$
A:=\hat{\rho} \circ\left(f^{(j)-1}\right)_{y}^{(o)} \circ \rho^{-1} .
$$

Then we have

$$
\begin{aligned}
& A \circ d_{x} f^{(j)}=\operatorname{id}_{T_{x} M}, \\
& d_{x} f^{(j)} \circ A=\operatorname{id}_{T_{y} E_{j}} .
\end{aligned}
$$

Namely, $A=\left(d_{x} f^{(j)}\right)^{-1}$ is determined independently of the choice of $(o)$. By its construction, $A=d_{y}\left(f^{(j)-1}\right)$ is well-defined. Thus $f^{(j)-1}$ is differentiable.

Since $f$ is the composition of differentiable map $f^{(j)}$ and smooth map $\pi_{j}, f$ and $f^{-1}$ are also differentiable.

Set $y_{j}:=y_{j}^{+1}$. Remark that $y_{j}$ can be taken to satisfy the following:

$$
\left\{\begin{array}{l}
\left|x_{j} y_{j}\right|=t \\
\angle S x y_{j} \geq \tilde{\angle} S x y_{j}>\pi-\delta^{\prime}-\theta(\varepsilon, s / t)
\end{array}\right.
$$

for all $x \in B\left(x_{j}, s\right)$.

Now, let us forget the construction of $f_{j}$ above. We will use the following notation:

$$
f_{j}:=f^{(j)}
$$

We set

$$
\begin{aligned}
Y_{j}(x) & :=\uparrow_{x}^{y_{j}} \in \Sigma_{x} M, \\
Z_{j}(x) & :=\frac{f_{j}\left(y_{j}^{+1}\right)-f_{j}(x)}{\left|f_{j}\left(y_{j}^{+1}\right)-f_{j}(x)\right|} \in E_{j},
\end{aligned}
$$

for all $x \in B\left(x_{j}, s\right)$.

We recall that $f_{j}$ is $\theta(\varepsilon)$-isometric on $B\left(x_{j}, t\right)$. It follows from (3.10) that we have

$$
d f_{j}\left(Y_{j}(x)\right)=Z_{j}(x)+\vec{v}(\theta(\varepsilon))
$$

for any $x \in B\left(x_{j}, 0.4 s\right)$. 
Since

$$
\angle_{x}\left(S^{\prime}, Y_{j}\right)+\angle_{x}\left(S^{\prime}, Y_{k}\right)+\angle_{x}\left(Y_{j}, Y_{k}\right) \leq 2 \pi
$$

we have

$$
\angle\left(Y_{j}(x), Y_{k}(x)\right)<2 \delta^{\prime}+\theta(\varepsilon)
$$

for all $x \in B\left(x_{j}, s\right) \cap B\left(x_{k}, s\right)$. Then, we have

$$
\begin{aligned}
d\left(Y_{j}, Y_{k}\right)^{2} & <2\left(1-\cos \left(2 \delta^{\prime}+\theta(\varepsilon)\right)\right) \\
& \leq 2 \delta^{\prime 2}+\theta(\varepsilon) .
\end{aligned}
$$

Therefore, we obtain

$$
\begin{aligned}
d f_{j}\left(Y_{k}(x)\right) & =d f_{j}\left(Y_{j}(x)\right)+\vec{v}\left(\sqrt{2} \delta^{\prime}+\theta(\varepsilon)\right) \\
& =Z_{j}(x)+\vec{v}\left(\sqrt{2} \delta^{\prime}+\theta(\varepsilon)\right) .
\end{aligned}
$$

Note that $Z_{j}$ is smooth on $V_{j} \subset E_{j}$. We define a smooth vector field $\tilde{W}_{j}$ on $\tilde{V}_{j} \subset N$ by

$$
\tilde{W}_{j}(x):=d \tilde{f}_{j}^{-1}\left(Z_{j}(x)\right) .
$$

We next prove the following.

Lemma 3.6. For any $x \in \tilde{V}_{j} \cap \tilde{V}_{k}$, we have

$$
\left|\tilde{W}_{j}(x)-\tilde{W}_{k}(x)\right|_{N}<4 \sqrt{2} \delta^{\prime}+\theta(\varepsilon) .
$$

Proof. At first, we see

$$
\begin{aligned}
\left|\tilde{W}_{j}-\tilde{W}_{k}\right|_{N}^{2} & =\sum_{\ell=1}^{N} \chi_{\ell}\left|d \tilde{f}_{\ell}\left(\tilde{W}_{j}-\tilde{W}_{k}\right)\right|_{E_{\ell}}^{2} \\
& =\sum_{\ell=1}^{N} \chi_{\ell}\left|d \tilde{F}_{\ell}^{j}\left(Z_{j}\right)-d \tilde{F}_{\ell}^{k}\left(Z_{k}\right)\right|_{E_{\ell}}^{2} .
\end{aligned}
$$

By (3.24), we have

$$
\begin{aligned}
d \tilde{F}_{\ell}^{k}\left(Z_{k}\right) & =d \tilde{F}_{\ell}^{k}\left(d f_{k}\left(Y_{k}\right)\right)+\vec{v}(\theta(\varepsilon)) \\
& =d f_{\ell}\left(Y_{k}\right)+\vec{v}(\theta(\varepsilon)) \\
& =Z_{\ell}+\vec{v}\left(2 \sqrt{2} \delta^{\prime}+\theta(\varepsilon)\right) .
\end{aligned}
$$

Therefore, Lemma 3.6 is proved.

We next define a smooth vector field $\tilde{W}$ on $N$ by

$$
\tilde{W}(x):=\sum_{j=1}^{N} \chi_{j}(x) \tilde{W}_{j}(x) .
$$

By Lemma 3.6, we have

$$
\begin{aligned}
\left|\tilde{W}-\tilde{W}_{j}\right| & =\left|\sum_{\ell} \chi_{\ell} \cdot \tilde{W}_{\ell}-\tilde{W}_{j}\right| \\
& \leq \sum_{\ell} \chi_{\ell}\left|\tilde{W}_{\ell}-\tilde{W}_{j}\right|<4 \sqrt{2} \delta^{\prime}+\theta(\varepsilon)
\end{aligned}
$$

on $\tilde{V}_{j} \subset N$. 
We consider an integral flow $\tilde{\Phi}$ of $\tilde{W}$, namely,

$$
\frac{d \tilde{\Phi}}{d t}(x, t)=\tilde{W}(\tilde{\Phi}(x, t)) .
$$

We now define a flow $\Phi$ on $U$ by

$$
\Phi(x, t):=f^{-1}(\tilde{\Phi}(f(x), t)) .
$$

Lemma 3.7. The conclusion (ii) of Theorem 3.2 holds:

$$
\begin{aligned}
\left.\frac{d}{d t}\right|_{t=0+} \operatorname{dist}_{S} \circ \Phi(x, t) & >1-5 \sqrt{\delta}-\theta(\varepsilon), \\
\left(\tilde{d}_{S}\right)^{\prime}(\tilde{W}(\tilde{x})) & >1-5 \sqrt{\delta}-\theta(\varepsilon)
\end{aligned}
$$

for all $x \in U$.

Proof. By Lemma 3.5 $f$ is differentiable. Therefore, the flow curve

$$
\Phi(x, \cdot): I_{x} \rightarrow U
$$

is differentiable for any $x \in U$. Then $x \in V_{j}$ for some $j$. Then, we have

$$
\begin{aligned}
\left.\frac{d}{d t}\right|_{t=0} d_{S} \circ \Phi(x, t) & =\left(d_{S} \circ f^{-1}\right)^{\prime}\left(\frac{d}{d t} f \circ \Phi(x, t)\right) \\
& =\left(d_{S} \circ f^{-1}\right)^{\prime}(\tilde{W}(f(x)))=\left(d_{S} \circ f_{j}^{-1}\right)^{\prime} \circ d \tilde{f}_{j}(\tilde{W}) \\
& =\left(d_{S} \circ f_{j}^{-1}\right)^{\prime} \circ d \tilde{f}_{j}\left(\tilde{W}_{j}+4 \sqrt{2} \vec{v}\left(\delta^{\prime}+\theta(\varepsilon)\right)\right) \\
& =\left(d_{S} \circ f_{j}^{-1}\right)^{\prime}\left(Z_{j}+4 \sqrt{2} \vec{v}\left(\delta^{\prime}+\theta(\varepsilon)\right)\right) \\
& =\left(d_{S} \circ f_{j}^{-1}\right)^{\prime}\left(d f_{j}\left(Y_{j}\right)+4 \sqrt{2} \vec{v}\left(\delta^{\prime}+\theta(\varepsilon)\right)\right) \\
& >d_{S}^{\prime}\left(Y_{j}\right)-4 \sqrt{2} \delta^{\prime}-\theta(\varepsilon) \\
& >1-\delta-4 \sqrt{2} \delta^{\prime}-\theta(\varepsilon) \\
& >1-5 \sqrt{\delta}-\theta(\varepsilon) .
\end{aligned}
$$

Lemma 3.8. The conclusion ( $i)$ of Theorem 3.2 holds: For any $x \in U, \Phi(x, t)$ is a $5 \sqrt{\delta}+\theta(\varepsilon)$-isometric embedding in $t \in I_{x}$. Here, $I_{x}:=\{t \in \mathbb{R} \mid \Phi(x, t) \in U\}$.

Proof. By the construction of $\tilde{W}$, we have $|\tilde{W}| \leq 1+\theta(\varepsilon)$. Indeed, for any $t, t^{\prime} \in I_{x}$ with $t<t^{\prime}$, we obtain

$$
\begin{aligned}
d\left(\tilde{\Phi}\left(f(x), t^{\prime}\right), \tilde{\Phi}(f(x), t)\right) & \leq \int_{t}^{t^{\prime}}|\tilde{W}(\tilde{\Phi}(f(x), s))| d s \\
& \leq|1+\theta(\varepsilon)|\left(t^{\prime}-t\right) .
\end{aligned}
$$

Then we have

$$
\begin{aligned}
\frac{d\left(\Phi\left(x, t^{\prime}\right), \Phi(x, t)\right)}{t^{\prime}-t} & =\frac{\left|\Phi\left(x, t^{\prime}\right), \Phi(x, t)\right|}{\left|\tilde{\Phi}\left(f(x), t^{\prime}\right), \tilde{\Phi}(f(x), t)\right|} \cdot \frac{\left|\tilde{\Phi}\left(f(x), t^{\prime}\right), \tilde{\Phi}(f(x), t)\right|}{t^{\prime}-t} \\
& \leq 1+\theta(\varepsilon) .
\end{aligned}
$$


By Lemma 3.7 for $t<t^{\prime}$ in $I_{x}$, we obtain

$$
\begin{aligned}
d\left(\Phi\left(x, t^{\prime}\right), \Phi(x, t)\right) & \geq d\left(S, \Phi\left(x, t^{\prime}\right)\right)-d(S, \Phi(x, t)) \\
& =\int_{t}^{t^{\prime}}\left(d_{S}\right)^{\prime}(W) d s \geq(1-5 \sqrt{\delta}-\theta(\varepsilon))\left(t^{\prime}-t\right) .
\end{aligned}
$$

This completes the proof of Lemma 3.8

Combining Lemmas 3.8 and 3.7, we obtain the conclusions of Theorem 3.2 ,

Definition 3.9. Let $M$ be an Alexandrov space, $f: M \rightarrow \mathbb{R}$ be a Lipschitz function and $\Phi: M \times \mathbb{R} \rightarrow M$ be a Lipschitz flow. Let $M^{\prime}$ be a subset of $M$. We say that $\Phi$ is gradient-like for $f$ on $M^{\prime}$ if there exists a constant $c>0$ such that for any $x \in M^{\prime}$ we have

$$
\liminf _{t \rightarrow 0} \frac{f(\Phi(x, t))-f(x)}{t}>c .
$$

We denote by

$$
\Phi \pitchfork f \text { on } M^{\prime}
$$

this situation.

In this notation, we obtained in Theorem 3.2 a gradient-like flow $\Phi$ for $\operatorname{dist}_{S}$ on $U(C)$ with a constant $c=1-5 \sqrt{\delta}-\theta(\varepsilon)$.

3.2. Flow and fibration. We will find a nice relation between Fibration Theorems 2.24 and 2.25 and Flow Theorem 3.2. We first recall an important property of Yamaguchi's fibration.

Proposition 3.10 (cf. Lemma 4.6 in $\mathrm{Y}$ conv $)$. Let $M$ and $X$ be Alexandrov spaces and $\pi: M \rightarrow X$ be a $\theta(\delta, \varepsilon)$-Lipschitz submersion as in Theorem 2.24. Let $(o)=\left(\varepsilon_{i}\right)$ be an arbitrary scale. We denote by $H_{x}$ a set of horizontal directions to the fiber $\pi^{-1}(\pi(x))$ at $x$. Then for any $x \in M$, the restriction of the blow-up

$$
\pi_{x}^{(o)} \circ \exp _{x}^{(o)}: H_{x} \rightarrow X_{\pi(x)}^{(o)}
$$

satisfies the following: For any $Y, Z \in H_{x}$, we have

$$
|| \pi_{x}^{(o)} \circ \exp _{x}^{(o)}(Y), \pi_{x}^{(o)} \circ \exp _{x}^{(o)}(Z)|-| Y, Z||<\theta(\delta, \varepsilon) .
$$

Here, the set of horizontal directions is defined in [Y conv, §4] as

$$
H_{x}:=\left\{\xi \in y_{x}^{\prime}|| x y \mid \geq \sigma\right\}
$$

for some small number $\sigma>0$ with $\varepsilon \ll \sigma$.

Proof of Proposition 3.10. We will use the following notation: $\theta$ denotes a variable constant $\theta(\delta, \varepsilon)$. We set $\bar{x}=\pi(x)$ for any $x \in M$.

Let us take $Y \in H_{x}$. By the definition of $H_{x}$, there is a point $y \in M$ such that

$$
|x y| \geq \sigma, \angle\left(y^{\prime}, Y\right)<\theta .
$$

Then, by Lemma 4.6 in $\mathrm{Y}$ conv, for any $\bar{Y} \in \bar{y}^{\prime} \subset \Sigma_{\bar{x}} X$, we have

$$
\frac{\left|\pi\left(\gamma_{Y}(t)\right), \gamma_{\bar{Y}}(t)\right|}{t}<\theta
$$


for any small $t>0$. Here, $\gamma_{\xi}$ denotes the geodesic from $\gamma_{\xi}(0)$ tangent to $\xi \in \Sigma_{\gamma(0)}$. Let $(o)=\left(\varepsilon_{i}\right)$ be an arbitrary scale. From (3.26), we have

$$
\left|\pi_{x}^{(o)} \circ \exp _{x}^{(o)}(Y), \exp _{\bar{x}}^{(o)}(\bar{Y})\right|=\lim _{\omega} \frac{\left|\pi\left(\gamma_{Y}\left(\varepsilon_{i}\right)\right), \gamma_{\bar{Y}}\left(\varepsilon_{i}\right)\right|}{\varepsilon_{i}}<\theta .
$$

We next take any $Z \in H_{x}$. Then there exists $z \in M$ such that

$$
|x z| \geq \sigma, \angle\left(z^{\prime}, Z\right)<\theta .
$$

Then, for any $\bar{Z} \in \bar{z}^{\prime} \subset \Sigma_{\bar{x}} X$, we have

$$
\left|\pi_{x}^{(o)} \circ \exp _{x}^{(o)}(Z), \exp _{\bar{x}}^{(o)}(\bar{Z})\right|<\theta .
$$

On the other hand, by Lemma 4.7 in $\mathrm{Y}$ conv, we have

$$
|\angle(Y, Z)-\angle(\bar{Y}, \bar{Z})|<\theta .
$$

It follows together with (3.26), (3.27) and (3.28) that we obtain

$$
\left\|\pi_{x}^{(o)} \circ \exp _{x}^{(o)}(Y), \pi_{x}^{(o)} \circ \exp _{x}^{(o)}(Z)|-| Y, Z\right\|<\theta .
$$

This completes the proof.

Theorem 3.11. For any $n \in \mathbb{N}$, there is a positive number $\epsilon=\epsilon_{n}$ satisfying the following: Let $M^{n}$ be an n-dimensional Alexandrov space without boundary with curvature $\geq-1$ and $p$ be a point of $M^{n}$. Let $X^{n-1}$ be an $(n-1)$ dimensional non-negatively curved Alexandrov space. Assume that $X$ is given by the Euclidean cone $K(\Sigma)$ over a closed Riemannian manifold $\Sigma$ of curvature $\geq 1$. If $d_{G H}\left((M, p),\left(X, p_{0}\right)\right)<\epsilon$, where $p_{0}$ is the origin of the cone $X$, then there exists a small $r=r_{p}>0$ such that a metric sphere $\partial B(p, r)$ is homeomorphic to an $S^{1}$-fiber bundle over $\Sigma$.

Proof. $d_{G H}\left((M, p),\left(X, p_{0}\right)\right)<\varepsilon$ implies $d_{G H}\left(B_{M}(p, 1 / \varepsilon), B_{X}\left(p_{0}, 1 / \varepsilon\right)\right)<\varepsilon$. Take a small number $r>0$ such that $r \ll 1 \ll 1 / \varepsilon$. Since $\Sigma$ is a closed Riemannian manifold, $A\left(p_{0} ; r / 2,2\right)$ is a Riemannian manifold $\approx \Sigma \times[r / 2,2]$ with boundary $\approx \Sigma \times\{r / 2,2\}$.

Let $C$ be an annulus $C:=A(p ; r / 2,2)$. Since $d_{G H}\left(C, A\left(p_{0} ; r / 2,2\right)\right)<\varepsilon, C$ is $(n-1, \varepsilon)$-strained. Since $M$ has no boundary points, Theorem 2.10 implies that $C$ is $(n, \theta(\varepsilon))$-strained. Therefore by Theorem 2.25 , there exists a $\theta(\varepsilon)$-Lipschitz submersion $\pi: M_{1} \rightarrow A\left(p_{0} ; r / 2,2\right)$ which is actually an $S^{1}$-fiber bundle. Here, $M_{1}$ is some closed domain in $M$ near $C$ containing $A(p ; r, 1)$.

Set $S:=\pi^{-1}\left(\partial B\left(p_{0}, r\right)\right)$. Let $\Phi=\Phi(x, t)$ be a gradient-like flow for $\operatorname{dist}_{p}$ obtained by Theorem 3.2 on an annulus around $p$.

We are going to prove

Lemma 3.12. The flow $\Phi$ is gradient-like for dist $_{p_{0}} \circ \pi$. Namely, we obtain the following:

$$
\liminf _{t \rightarrow 0+} \frac{\operatorname{dist}_{p_{0}} \circ \pi \circ \Phi(x, t)-\operatorname{dist}_{p_{0}} \circ \pi(x)}{t}>1-\theta(\varepsilon)
$$

for any $x \in M_{1}$.

If it is proved, then $S$ is homeomorphic to $\partial B(p, r)$ by a standard flow argument. 
Proof of Lemma 3.12, Let us set $\bar{x}:=\pi(x)$ for any $x \in M_{1}$. We set

$$
V:=\left.\frac{d}{d t}\right|_{t=0+} \Phi(x, t) \in T_{x} M .
$$

By Theorem 3.2 (ii), we have

$$
V \doteqdot \nabla \operatorname{dist}_{p}
$$

and $|V| \doteqdot 1$. Here, $A \doteqdot A^{\prime}$ means that $d\left(A, A^{\prime}\right)<\theta(\varepsilon)$.

We set $\xi:=V /|V|$ and recall that $\xi \in H_{x}$. It follows together with (3.27) that there exists $q \in M$ with $|x q| \geq \sigma$ such that any $\bar{\xi} \in \bar{q}^{\prime} \subset \Sigma_{\bar{x}} X$ satisfies

$$
\pi_{x}^{(o)} \circ \exp _{x}^{(o)}(\xi) \doteqdot \exp _{\bar{x}}^{(o)}(\bar{\xi})
$$

for each scale $(o)$.

Let us take $\eta \in p_{x}^{\prime} \subset \Sigma_{x} M$. Then we have

$$
\angle(\xi, \eta)>\pi-\theta(\varepsilon) .
$$

Since $\eta \in H_{x}$, there exists $\bar{\eta} \in \Sigma_{\bar{x}} X$ such that

$$
\pi_{x}^{(o)} \circ \exp _{x}^{(o)}(\eta) \doteqdot \exp _{\bar{x}}^{(o)}(\bar{\eta}) .
$$

By Proposition 3.10, we obtain

$$
\angle(\bar{\xi}, \bar{\eta})>\pi-\theta(\varepsilon) .
$$

On the other hand, from Lemma 4.3 in $\mathrm{Y} \mathrm{conv}, \pi$ is $\theta(\varepsilon)$-close to an $\varepsilon$ approximation from $(M, p)$ to $\left(X, p_{0}\right)$. This implies

$$
\tilde{\angle} \bar{q} \bar{x} p_{0}>\pi-\theta(\varepsilon) \text {. }
$$

We take an arbitrary direction $\bar{\zeta} \in p_{0}^{\prime} \subset \Sigma_{\bar{x}} X$. Then, we have

$$
\angle(\bar{\zeta}, \bar{\xi})>\pi-\theta(\varepsilon)
$$

By (3.30) and (3.31), we have

$$
\bar{\xi} \doteqdot \nabla \operatorname{dist}_{p_{0}}
$$

Summarizing the above arguments, we obtain

$$
\begin{aligned}
\lim _{\omega} \frac{d\left(p_{0}, \pi \circ \Phi\left(x, \varepsilon_{i}\right)\right)-d\left(p_{0}, \bar{x}\right)}{\varepsilon_{i}} & =\left(\operatorname{dist}_{p_{0}}\right)_{\bar{x}}^{\prime} \circ\left(\exp _{\bar{x}}^{(o)}\right)^{-1} \circ \pi_{x}^{(o)} \circ \exp _{x}^{(o)}(V) \\
& \doteqdot\left(\operatorname{dist}_{p_{0}}\right)_{\bar{x}}^{\prime}(\bar{\xi}) \\
& \doteqdot\left(\operatorname{dist}_{p_{0}}\right)_{\bar{x}}^{\prime}\left(\nabla \operatorname{dist}_{p_{0}}\right) \\
& =1 .
\end{aligned}
$$

It follows from Lemma 2.14 that we obtain (3.29).

As mentioned above, by Lemma 3.12 , we have $\partial B(p, r) \approx S$. This completes the proof of Theorem 3.11

Remark 3.13. Kapovitch proved a statement similar to Theorem 3.11 for collapsing Riemannian manifolds $M$ ([Kap Rest, Theorem 7.1]).

Perelman and Petrunin proved the existence and uniqueness of a gradient flow of any semiconcave function, especially of any distance function ( Pet QG, PP QG $)$. Note that the gradient "flow" is not a flow in the sense of Definition 3.1, because the gradient flow is defined on $M \times[0, \infty)$. 
Remark 3.14. One might ask why we do not use the gradient flow of a distance function to prove Theorem 3.11. The reason is that the gradient flow may not be injective.

For instance, we consider the cone $X=K\left(S_{\theta}^{1}\right)$ over a circle $S_{\theta}^{1}$ with length $\theta<2 \pi$. $X$ is expressed by the quotient of a set

$$
X_{0}=\left\{r e^{i t} \in \mathbb{C} \mid r \geq 0, t \in[0, \theta]\right\}
$$

by a relation $r \sim r e^{i \theta}$ for $r \geq 0$. By $\left[r e^{i t}\right] \in X$ we denote the equivalent class of $r e^{i t} \in X_{0}$. We fix $r>0$ and take $p:=r e^{i \theta / 2}$. Let $a>0$ be a sufficiently small number such that $S_{a} \cap \partial X_{0}=\emptyset$. Here, we denote by $S_{a}$ the circle centered at $p$ with radius $a$ in $\mathbb{C}$. We take $b$ with $a<b<r$ such that $S_{b} \cap \partial X_{0} \neq \emptyset$ and take $x_{1}, x_{2}$ with $x_{1} \neq x_{2}$ in $S_{b} \cap \partial X_{0}$ near $p$. Then $\left[x_{1}\right]=\left[x_{2}\right]$ in $X$. We put points $y_{i} \in p x_{i} \cap S_{a}$ in $X_{0}$ and set geodesics $\gamma_{i}:=\left[y_{i}\right]\left[x_{i}\right]$ in $X$ for $i=1,2$. In particular, $\gamma_{i}(i=1,2)$ are the gradient curves for $d_{[p]}$ in $X$. This case says that $d_{[p]}$-flow does not injectively send $\left[S_{a}\right]:=\left\{[z] \in X \mid x \in S_{a}\right\}$ to $\left[S_{b}\right]$.

\subsection{Flow arguments.}

Theorem 3.15. For a positive integer $n$, there is a positive constant $\varepsilon_{n}$ satisfying the following: Let $M^{n}$ be an $n$-dimensional Alexandrov space with curvature $\geq-1$. Let $A_{1}, A_{2}, \ldots, A_{m} \subset M$ be closed subsets and $C \subset M$ be an $(n, \varepsilon)$-strained compact subset with $A_{i} \cap C=\emptyset$ for all $i=1,2, \ldots, m$ and for $\varepsilon \leq \varepsilon_{n}$. Suppose the following: For each $x \in C$ and $1 \leq i \leq m$, there is a point $w=w(x) \in M$ such that

$$
\angle_{x}\left(A_{i}^{\prime}, w^{\prime}\right)>\pi-\delta .
$$

Here, $c(<\pi / 2)$ is a positive constant bigger than some constant. Then there exist an open neighborhood $U$ of $C$ and a Lipschitz flow $\Phi$ on $M$ such that

$$
\left.\frac{d}{d t}\right|_{t=0+} d_{A_{i}}(\Phi(x, t))>1-5 \delta-\theta(\varepsilon)
$$

for all $i=1, \ldots, m$ and $x \in U$.

Proof. We can show the following: There exists a precompact open neighborhood $U$ of $C$ such that each $x \in U$ is $(n, \theta(\varepsilon))$-strained, and there is a point $w=w(x) \in M$ such that

$$
\begin{aligned}
|x w(x)| & >\ell, \\
\tilde{\angle} A_{i} y w(x) & >\pi-\delta-\theta(\varepsilon),
\end{aligned}
$$

for all $y \in B(x, r)$ and $i=1, \ldots, m$. Here, $r$ and $\ell$ are positive numbers with $r \ll \ell$.

Since $U$ is $(n, \theta(\varepsilon))$-strained, there is a smooth approximation $f: U \rightarrow N$ which is a $\theta(\varepsilon)$-isometry for some Riemannian manifold $N$. By an argument similar to the proof of Theorem 3.2 , we can construct a smooth vector field $\tilde{W}$ and its integral flow $\tilde{\Phi}$ on $N$ such that

$$
\begin{aligned}
\left.\frac{d}{d t}\right|_{t=0+} \operatorname{dist}_{A_{i}} \circ f^{-1}(\tilde{\Phi}(x, t)) & =\operatorname{dist}_{A_{i}}^{\prime} \circ d f^{-1}(\tilde{W}) \\
& >1-5 \delta-\theta(\varepsilon) .
\end{aligned}
$$

Then, the pull-back flow $\Phi_{t}:=\tilde{\Phi}_{t} \circ f$ satisfies the conclusion of Theorem 3.15, 
Corollary 3.16. Let $M^{n}, A_{1}, A_{2}, \ldots, A_{m}$ and $C$ be the same as in the assumption of Theorem 3.15 and satisfy the following: All $d_{A_{i}}$ are $(1-\delta)$-regular at $x, m \leq n$ and

$$
\left|\measuredangle_{x}\left(A_{i}^{\prime}, A_{j}^{\prime}\right)-\pi / 2\right|<\mu
$$

for any $x \in C$ and $1 \leq i \neq j \leq m$.

If $\nu:=\delta+\mu$ is smaller than some constant depending on $m$, then there are a Lipschitz flow $\Phi$ and a neighborhood $U$ of $C$ satisfying the following:

$$
\left.\frac{d}{d t}\right|_{t=0+} d_{A_{i}}(\Phi(x, t))>1-5 \sqrt{\delta}-\theta(\varepsilon)-\theta_{m}(\nu)
$$

for any $x \in U$ and $i=1, \ldots, m$.

Proof. Let us consider a smooth approximation

$$
f: U \rightarrow N
$$

for some neighborhood $U$ of $C$ and a Riemannian manifold $N$. By Lemmas 3.7 and 3.8. we obtain smooth vector fields $\tilde{W}_{i}$ on $N$ such that

$$
\begin{aligned}
\left|\tilde{W}_{i}\right| & \leq 1+\theta(\varepsilon), \\
\left(d_{A_{i}}\right)^{\prime}\left(W_{i}\right) & >1-5 \sqrt{\delta}-\theta(\varepsilon)
\end{aligned}
$$

on $N$ for all $i=1, \cdots, m$. Here, $W_{i}:=d f^{-1}\left(\tilde{W}_{i}\right)$.

Let us define $\varphi_{m}(\nu) \in(\pi / 2, \pi)$ by

$$
\cos \varphi_{m}(\nu)=\frac{1-(m-1) \cos (\pi / 2-\nu)}{\sqrt{m} \sqrt{1+(m-1) \cos (\pi / 2-\nu)}} .
$$

Note that $\cos \varphi_{m}(\nu) \rightarrow 1 / \sqrt{m}$ as $\nu \rightarrow 0$.

Let us consider a vector field

$$
\tilde{W}:=\left(\tilde{W}_{1}+\tilde{W}_{2}+\cdots+\tilde{W}_{m}\right) /\left|\tilde{W}_{1}+\tilde{W}_{2}+\cdots+\tilde{W}_{m}\right| .
$$

Since $\left|\angle\left(A_{i}^{\prime}, A_{j}^{\prime}\right)-\pi / 2\right|<\mu$, we have

$$
\left|\angle\left(W_{i}, W_{j}\right)\right|<10 \delta+\mu+\theta(\varepsilon) .
$$

Putting $W:=d f^{-1}(\tilde{W})$, we obtain

$$
\cos \angle\left(W_{i}, W\right) \geq \cos \left(\varphi_{m}(\nu)+\theta(\varepsilon)\right)
$$

for $\nu=10 \delta+\mu$. Then we have

$$
\begin{aligned}
\left(d_{A_{i}}\right)^{\prime}(W) & \geq\left(d_{A_{i}}\right)^{\prime}\left(W_{i}\right)-\left|W, W_{i}\right| \\
& \geq 1-5 \sqrt{\delta}-\cos \left(\varphi_{m}(\nu)\right)-\theta(\varepsilon) .
\end{aligned}
$$

We consider the gradient flow $\Phi$ of the vector field $W$ on $U$, which is the desired flow. 


\section{The Case that $\operatorname{dim} X=2$ and $\partial X=\emptyset$}

In this and the next sections, we study the topologies of three-dimensional closed Alexandrov spaces which collapse to Alexandrov surfaces. First, we exhibit examples of three-dimensional Alexandrov spaces (which are closed or open) collapsing to Alexandrov surfaces.

We denote a circle of length $\varepsilon$ by $S_{\varepsilon}^{1}$. We often regard $S_{\varepsilon}^{1}$ as $\{x \in \mathbb{C} \mid\|x\|=$ $\varepsilon / 2 \pi\}$, and $\bar{x}$ denotes the complex conjugate for $x \in \mathbb{C}$.

Example 4.1. Recall that $M_{\mathrm{pt}}$ is obtained by the quotient space $M_{\mathrm{pt}}:=S^{1} \times$ $\mathbb{R}^{2} /(x, y) \sim(\bar{x},-y) . M_{\mathrm{pt}}$ have collapsing metrics $d_{\varepsilon}$ and $\rho_{\varepsilon}$ as follows.

Recall that a collapsing metric provided Example 1.2. The quotient $\left(M_{\mathrm{pt}}, d_{\varepsilon}\right):=$ $S_{\varepsilon}^{1} \times \mathbb{R}^{2} /(x, y) \sim(\bar{x},-y)$ has a metric $d_{\varepsilon}$ of non-negative curvature collapsing to $K\left(S_{\pi}^{1}\right)=\mathbb{R}^{2} / y \sim-y$ as $\varepsilon \rightarrow 0$.

We consider an isometry defined by

$$
K\left(S_{\varepsilon}^{1}\right) \ni[t, v] \mapsto[t,-v] \in K\left(S_{\varepsilon}^{1}\right) .
$$

Here, $t \geq 0$ and $v \in S_{\varepsilon}^{1}$. Note that $K\left(S_{\varepsilon}^{1}\right)$ collapses to $\mathbb{R}_{+}$as $\varepsilon \rightarrow 0$. We consider a metric $\rho_{\varepsilon}$ on $M_{\mathrm{pt}}$ of non-negative curvature defined by taking the quotient of the direct product $S^{1} \times K\left(S_{\varepsilon}^{1}\right)$ :

$$
\left(M_{\mathrm{pt}}, \rho_{\varepsilon}\right):=S^{1} \times K\left(S_{\varepsilon}^{1}\right) /(x, t, v) \sim(\bar{x}, t,-v) .
$$

Then, $\left(M_{\mathrm{pt}}, \rho_{\varepsilon}\right)$ collapses to $[0, \pi] \times \mathbb{R}_{+}$as $\varepsilon \rightarrow 0$. Here, $[0, \pi]$ is provided as $S^{1} / x \sim \bar{x}$.

Example 4.2. Let $\Sigma\left(S_{\varepsilon}^{1}\right)$ be the spherical suspension of $S_{\varepsilon}^{1}$, which has curvature $\geq 1$. Any point in $\Sigma\left(S_{\varepsilon}^{1}\right)$ is expressed as $[t, v]$ parametrized by $t \in[0, \pi]$ and $v \in S_{\varepsilon}^{1}$. We consider an isometry

$$
\alpha: \Sigma\left(S_{\varepsilon}^{1}\right) \ni[t, v] \mapsto[\pi-t,-v] \in \Sigma\left(S_{\varepsilon}^{1}\right) .
$$

Then, we obtain a metric $d_{\varepsilon}$ on $P^{2}$ of curvature $\geq 1$ defined by taking the quotient $\Sigma\left(S_{\varepsilon}^{1}\right) /\langle\alpha\rangle$. We set $P_{\varepsilon}^{2}:=\left(P^{2}, d_{\varepsilon}\right)$. Note that $P_{\varepsilon}^{2}$ collapses to $[0, \pi / 2]$ as $\varepsilon \rightarrow 0$. Then, $K\left(P_{\varepsilon}^{2}\right)$ collapses to $K([0, \pi / 2]) \equiv \mathbb{R}_{+} \times \mathbb{R}_{+}$as $\varepsilon \rightarrow 0$.

Remark that $K\left(P_{\varepsilon}^{2}\right)$ is isometric to the quotient space $\mathbb{R} \times K\left(S_{\varepsilon}^{1}\right) /\langle\sigma\rangle$ defined as follows: Let $\sigma$ be an involution defined by

$$
\sigma(x, t v) \mapsto(-x, t(-v))
$$

for $x \in \mathbb{R}, t \geq 0$ and $v \in S_{\varepsilon}^{1}$. We sometimes use this expression in the paper.

Example 4.3. Let us consider the direct product $S^{1} \times \Sigma\left(S_{\varepsilon}^{1}\right)$ and an isometry

$$
\beta: S^{1} \times \Sigma\left(S_{\varepsilon}^{1}\right) \ni(x, t, v) \mapsto(\bar{x}, t,-v) \in S^{1} \times \Sigma\left(S_{\varepsilon}^{1}\right) .
$$

Then, the quotient space $N_{\varepsilon}:=S^{1} \times \Sigma\left(S_{\varepsilon}^{1}\right) /\langle\beta\rangle$ has non-negative curvature, and $N_{\varepsilon}$ collapses to $[0, \pi] \times[0, \pi]$ as $\varepsilon \rightarrow 0$.

Let us start the proof of Theorem 1.3 .

Proof of Theorem 1.3 . Fix a sufficiently small $\delta>0$. Then there are only finitely many $(2, \delta)$-singular points $x_{1}, \ldots, x_{k}$ in $X$. For sufficiently small $r>0$, consider the set $X^{\prime}:=X-\left(U\left(x_{1}, r\right) \cup \cdots \cup U\left(x_{k}, r\right)\right)$. By Theorem 2.10, there exists a $(3, \theta(i, \delta))$-strained closed domain $M_{i}^{\prime} \subset M_{i}$ which is converging to $X^{\prime}$. From Theorem 2.25, we may assume that there exists a circle fiber bundle $\pi_{i}^{\prime}: M_{i}^{\prime} \rightarrow X^{\prime}$ which is a $\theta(i, \delta)$-almost Lipschitz submersion. Here, $\theta(i, \delta)$ is a positive constant 
such that $\lim _{i \rightarrow \infty, \delta \rightarrow 0} \theta(i, \delta)=0$. We fix a large $i$ and use the notation $\theta(\delta)=\theta(i, \delta)$ for simplicity.

Fix any $(2, \delta)$-singular point $p \in\left\{x_{1}, \ldots, x_{k}\right\} \subset X$ and take a sequence $p_{i} \in$ $M_{i}$ converging to $p$. Since the Flow Theorem implies that $B\left(p_{i}, r\right)$ is not contractible, applying the rescaling argument Theorem 2.27, we have points $\hat{p}_{i} \in$ $B\left(p_{i}, r\right)$ with $d\left(p_{i}, \hat{p}_{i}\right) \rightarrow 0$ and a scaling constant $\delta_{i}$ such that any limit space $\left(Y, y_{0}\right)$ of $\lim _{i \rightarrow \infty}\left(\frac{1}{\delta_{i}} B\left(\hat{p}_{i}, r\right), \hat{p}_{i}\right)$ is a three-dimensional open Alexandrov space of non-negative curvature. We may assume that $p_{i}=\hat{p}_{i}$. We denote by $S$ a soul of $Y$. By Theorem 2.27, we have $\operatorname{dim} S \leq 1$.

From Theorem 3.11, the boundary $\partial B\left(p_{i}, r\right)$ is homeomorphic to a torus $T^{2}$ or a Klein bottle $K^{2}$. It follows from the Soul Theorem 2.59 and the Stability Theorem 2.34 that $B\left(p_{i}, r\right)$ is homeomorphic to the orbifold $B(\mathrm{pt})$ if $\operatorname{dim} S=0$ or a solid torus $S^{1} \times D^{2}$ or a solid Klein bottle $S^{1} \tilde{\times} D^{2}$ if $\operatorname{dim} S=1$.

We first consider the case of $\operatorname{dim} S=1$; namely, $S$ is a circle. In this case, we obtain the following conclusion.

Lemma 4.4. If $\operatorname{dim} S=1$, then $B\left(p_{i}, r\right)$ is homeomorphic to $S^{1} \times D^{2}$.

Proof. Put $B_{i}:=B\left(p_{i}, r\right), B:=B(p, r)$ and $\varepsilon_{i}:=d_{G H}\left(B_{i}, B\right)$. Suppose that $B_{i}$ is homeomorphic to a solid Klein bottle $S^{1} \tilde{\times} D^{2}$. Take $r_{i} \rightarrow 0$ with $\varepsilon_{i} / r_{i} \rightarrow 0$ such that $\lim \left(\frac{1}{r_{i}} B_{i}, p_{i}\right)=\left(T_{p} X, o\right)$. Let $\pi_{i}: \tilde{B}_{i} \rightarrow B_{i}$ be a universal covering and $\tilde{p}_{i} \in \pi_{i}^{-1}\left(p_{i}\right)$. Let $\Gamma_{i} \cong \mathbb{Z}$ be the deck transformation group of $\pi_{i}$. Passing to a subsequence, we have a limit triple $(Z, z, G)$ of a sequence of triples of pointed spaces and isometry groups $\left(\frac{1}{r_{i}} \tilde{B}_{i}, \tilde{p}_{i}, \Gamma_{i}\right)$ in the equivariant pointed Gromov-Hausdorff topology (cf. [FY]). $Z$ is an Alexandrov space of non-negative curvature because of $r_{i} \rightarrow 0$, and $G$ is abelian. Note that $Z / G=\lim \left(\frac{1}{r_{i}} B_{i}, p_{i}\right)=\left(T_{p} X, o\right)$. Using the $G$-action, we find a line in $Z$ ( $[\mathrm{ChGr})$. Then, by the Splitting Theorem, there is some non-negatively curved Alexandrov space $Z_{0}$ such that $Z$ is isometric to the product $\mathbb{R} \times Z_{0}$. We may assume that $Z_{0}$ is a cone by taking a suitable rescaling $\left\{r_{i}\right\}$. We denote by $G_{0}$ the identity component of $G$. By [FY, Lemma 3.10], there is a subgroup $\Gamma_{i}^{0}$ of $\Gamma_{i}$ such that:

(1) $\left(\frac{1}{r_{i}} \tilde{B}_{i}, \tilde{p}_{i}, \Gamma_{i}^{0}\right)$ converges to $\left(Z, z, G_{0}\right)$.

(2) $\Gamma_{i} / \Gamma_{i}^{0} \cong G / G_{0}$ for large $i$.

Since $\operatorname{dim} T_{p} X=2$ and $\operatorname{dim} Z=3$, we have $\operatorname{dim} G=1$. This implies $G \cong \mathbb{R} \times H$ for some finite abelian group $H$. Since $T_{p} X=Z_{0} / H, H$ must be cyclic. Here, $G$ action is component-wise: $G_{0} \cong \mathbb{R}$ acts by translation of the line $\mathbb{R}$ and $H$ acts on $Z_{0}$ independently. By Stability Theorem $2.34, Z$ is simply-connected. Therefore, $Z_{0}$ is homeomorphic to $\mathbb{R}^{2}$.

Take a generator $\gamma_{i}$ of $\Gamma_{i}$. From our assumption, $\gamma_{i}$ is orientation reversing on $\tilde{B}_{i}$. Consider $\Gamma_{i}^{\prime}:=\left\langle\gamma_{i}^{2}\right\rangle \cong \mathbb{Z}$. Then $\Gamma_{i}^{\prime}$ acts on $\tilde{B}_{i}$ preserving orientation. Taking a subsequence, we have a limit triple $\left(Z, z, G^{\prime}\right)$ of a sequence $\left\{\left(\frac{1}{r_{i}} \tilde{B}_{i}, \tilde{p}_{i}, \Gamma_{i}^{\prime}\right)\right\}$. By an argument similar to the above, $G^{\prime} \cong \mathbb{R} \times H^{\prime}$ for some finite cyclic group $H^{\prime}$. Let $\lim _{i \rightarrow \infty} \gamma_{i}=\gamma_{\infty} \in G$, which implies that $\gamma_{i}\left(x_{i}\right) \rightarrow \gamma_{\infty}\left(x_{\infty}\right)$ under the Gromov-Hausdorff convergence $\frac{1}{r_{i}} \tilde{B}_{i} \ni x_{i} \rightarrow x_{\infty} \in Z$. Then $\gamma_{\infty}$ is represented by $(0, \phi) \in \mathbb{R} \times H$. Then, for large $i$, we have

$$
Z / G=\left(Z / G^{\prime}\right) /\left(G / G^{\prime}\right)=\left(Z_{0} / H^{\prime}\right) /\langle[\phi]\rangle=T_{p} X
$$


Since $Z_{0} / H^{\prime}$ is the flat cone over a circle or an interval, and $[\phi] \in H / H^{\prime}$ acts on a $Z_{0} / H^{\prime}$ reversing orientation, $\left(Z_{0} / H^{\prime}\right) /\langle\phi\rangle$ cannot be $T_{p} X$. This is a contradiction.

By Lemma 4.4 $B\left(p_{i}, r / 2\right)$ must be homeomorphic to a solid torus. From Flow Theorem 3.2. $\left(\pi_{i}^{\prime}\right)^{-1}(\partial B(p, r))$ and $\partial B\left(p_{i}, r / 2\right)$ bound a closed domain homeomorphic to $T^{2} \times[0,1]$, and this provides a circle fiber structure on $\partial B\left(p_{i}, r / 2\right)$. By [SY00, Lemma 4.4], it extends to a topological Seifert structure on $B\left(p_{i}, r / 2\right)$ over $B(p, r / 2)$ which is compatible to the circle bundle structure on $A\left(p_{i} ; r / 2, r\right)$.

In the case of $\operatorname{dim} S=0, B_{i}$ is homeomorphic to $B(\mathrm{pt})$. We must prove that

Lemma 4.5. If $\operatorname{dim} S=0$, then $B_{i}$ has the structure of circle fibration with a singular arc fiber satisfying:

(1) it is isomorphic to the standard fiber structure on $B(p t)=S^{1} \times D^{2} / \mathbb{Z}_{2}$;

(2) it is compatible to the structure of circle fiber bundle $\pi_{i}^{\prime}$ near the boundary.

Proof. Recall that $B(p t)=S^{1} \times D^{2} / \mathbb{Z}_{2}$, where $\mathbb{Z}_{2}$-action on $S^{1} \times D^{2}$ is given by the involution $\hat{\sigma}$ defined by $\hat{\sigma}(x, y)=(\bar{x},-y)$. Let $p_{+}:=(1,0), p_{-}:=(-1,0)$ be the fixed points of $\hat{\sigma}$. Putting $\hat{U}:=S^{1} \times D^{2} \backslash\left\{p_{+}, p_{-}\right\}$, and $U:=\hat{U} / \mathbb{Z}_{2}$, let $\hat{\pi}: \hat{U} \rightarrow U$ be the projection map. Fix a homeomorphism $f_{i}: S^{1} \times D^{2} / \mathbb{Z}_{2} \rightarrow B_{i}$, and set $U_{i}:=f_{i}(U)$. Take a $\mathbb{Z}_{2}$-covering $\hat{\pi}_{i}: \hat{U}_{i} \rightarrow U_{i}$ such that there is a homeomorphism $\hat{f}_{i}: \hat{U} \rightarrow \hat{U}_{i}$ together with the following commutaitve diagram:

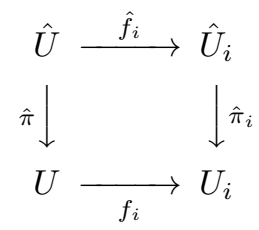

Consider the length-metric on $\hat{U}_{i}$ induced from that of $U_{i}$ via $\hat{\pi}_{i}$, and the lengthmetrics of $U$ and $\hat{U}$ for which both $f_{i}$ and $\hat{f}_{i}$ become isometries. Note that $U_{i}=$ $\hat{U}_{i} / \hat{\sigma}_{i}$, where $\hat{\sigma}_{i}:=\hat{f}_{i} \circ \hat{\sigma} \circ\left(\hat{f}_{i}\right)^{-1}$. $\hat{\sigma}_{i}$ extends to an isometry on the completion $\hat{B}_{i}$ of $\hat{U}_{i}$. Let $\hat{\pi}_{i}: \hat{B}_{i} \rightarrow B_{i}$ also denote the the projection. Then we have the following commutative diagram:

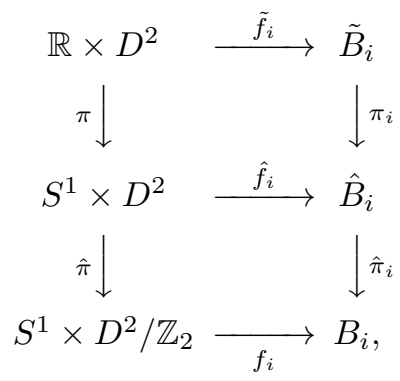

where $\pi_{i}: \tilde{B}_{i} \rightarrow \hat{B}_{i}$ is the universal covering, and $\tilde{f}_{i}$ is an isometry covering $\hat{f}_{i}$. Here we consider the metric on $\mathbb{R} \times D^{2}$ induced by that of $S^{1} \times D^{2}$. Let $\sigma, \lambda: \mathbb{R} \times D^{2} \rightarrow \mathbb{R} \times D^{2}$ be defined as

$$
\sigma(x, y)=(-x,-y), \lambda(x, y)=(x+1, y) .
$$

Since $\sigma$ covers $\hat{\sigma}, \sigma$ is an isometry. Put

$$
\sigma_{i}:=\tilde{f}_{i} \circ \sigma \circ\left(\tilde{f}_{i}\right)^{-1}, \lambda_{i}:=\tilde{f}_{i} \circ \lambda \circ\left(\tilde{f}_{i}\right)^{-1} .
$$


From construction, the group $\Lambda_{i}$ generated by $\lambda_{i}$ is the deck transformation group of $\pi_{i}: \tilde{B}_{i} \rightarrow \hat{B}_{i}$. Let $\Lambda$ be the group generated by $\lambda$. Let $\Gamma_{i}$ (resp. $\Gamma$ ) be the group generated by $\sigma_{i}$ and $\Lambda_{i}$ (resp. by $\sigma$ and $\Lambda$ ). Obviously we have an isomorphism $\left(\Gamma_{i}, \Lambda_{i}\right) \simeq(\Gamma, \Lambda)$. Note that

$$
\sigma \lambda \sigma^{-1}=\lambda^{-1}
$$

Let us consider the limit of the action of $\left(\Gamma_{i}, \Lambda_{i}\right)$ on $\tilde{B}_{i}$. We may assume that $\left(\tilde{B}_{i}, \tilde{p}_{i}, \Gamma_{i}, \Lambda_{i}\right)$ converges to $\left(Z, z_{0}, \Gamma_{\infty}, \Lambda_{\infty}\right)$, where $Z=\mathbb{R} \times L, \Lambda_{\infty}=\mathbb{R} \times H, L$ is a flat cone and $H$ is a finite cyclic group acting on $L$. Let $\sigma_{\infty} \in \Gamma_{\infty}$ and $\lambda_{\infty} \in \Lambda_{\infty}$ be the limits of $\sigma_{i}$ and $\lambda_{i}$ under the above convergence. Note that $\sigma_{\infty}: \mathbb{R} \times L \rightarrow \mathbb{R} \times L$ can be expressed as $\sigma_{\infty}(x, y)=\left(-x, \sigma_{\infty}^{\prime}(y)\right)$, where $\sigma_{\infty}^{\prime}$ is a rotation of angle $\ell / 2$ and $\ell$ is the length of the space of directions at the vertex of the cone $L$. Note that $T_{p} X=(L / H) / \sigma_{\infty}^{\prime}$.

As discussed above, from the action of $H$ on $L$, we can put a Seifert fibered torus structure on $\partial \hat{B}_{i}$. Namely, if $\lambda_{\infty}\left(r e^{i \theta}\right)=r e^{i(\theta+\nu \ell / \mu)}$, then $\partial \hat{B}_{i}$ has a Seifert fibered torus structure of type $(\mu, \nu)$ that is $\hat{\sigma}_{i}$-invariant (see [SY00, Lemma 4.4]). From (4.3), we have $\sigma_{\infty} \lambda_{\infty} \sigma_{\infty}=\lambda_{\infty}^{-1}$. This yields that $\lambda_{\infty}^{2}=1$. Thus $(\mu, \nu)$ is equal to $(1,1)$ or $(2,1)$.

We shall show $(\mu, \nu)=(1,1)$ and extend the fiber structure on $\partial \hat{B}_{i}$ to a $\hat{\sigma}_{i^{-}}$ invariant fiber structure on $\hat{B}_{i}$ which projects down to the generalized Seifert bundle structure on $B_{i}$.

Let $B$ and $\hat{B}$ be the $r$-balls in the cone $T_{p} X=(L / H) / \sigma_{\infty}^{\prime}$ and $L / H$ around the vertices $o_{p}$ and $\hat{o}_{p}$ respectively. Consider the metric annuli

$$
A:=A\left(o_{p} ; r / 4, r\right), \hat{A}:=A\left(\hat{o}_{p} ; r / 4, r\right) .
$$

Applying the equivariant Fibration Theorem (Theorem 18.4 in Y 4-dim), we have a $\mathbb{Z}_{2}$-equivariant $S^{1}$-fibration $\hat{g}_{i}: \hat{A}_{i} \rightarrow \hat{A}$ for some closed domain $\hat{A}_{i}$ of $\hat{B}_{i}$, which gives rise to an $S^{1}$-fibration $g_{i}: A_{i} \rightarrow A$ for some closed domain $A_{i}$ of $B_{i}$.

We denote by $B\left(\pi_{i}^{\prime}\right)$ and $B\left(g_{i}\right)$ the closed domain bounded by $\left(\pi_{i}^{\prime}\right)^{-1}(S(p, r))$ and $\left(g_{i}\right)^{-1}\left(S\left(o_{p}, r / 2\right)\right)$ respectively, and set

$$
A\left(\pi_{i}^{\prime}, g_{i}\right):=\overline{B\left(\pi_{i}^{\prime}\right) \backslash B\left(g_{i}\right)} .
$$

By Flow Theorem 3.2, there is a Lipschitz flow $\Phi: \partial B\left(\pi_{i}^{\prime}\right) \times[0,1] \rightarrow A\left(\pi_{i}^{\prime}, g_{i}\right)$ such that $\Phi(x, 0)=x$. Let $\Phi_{1}: \partial B\left(\pi_{i}^{\prime}\right) \rightarrow \partial B\left(g_{i}\right)$ be the homeomorpshism defined by $\Phi_{1}(x)=\Phi(x, 1)$. Obviously the $\pi_{i}^{\prime}$-fibers of $\left(\pi_{i}^{\prime}\right)^{-1}(S(p, r))$ and the $\left(\Phi_{1}\right)^{-1}$. images of $g_{i}$-fibers of $\left(g_{i}\right)^{-1}\left(S\left(o_{p}, r / 2\right)\right)$ are isotopic to each other. Namely, we have an isotopy $\varphi_{t}$ of $\partial B\left(\pi_{i}\right), 0 \leq t \leq 1$, such that $\varphi_{0}=i d$ and $\varphi_{1}$ sends every $\pi_{i}^{\prime}$-fiber to the $\left(\Phi_{1}\right)^{-1}$-image of a $g_{i}$-fiber. Define $\Psi: A\left(\pi_{i}^{\prime}, g_{i}\right) \rightarrow A\left(\pi_{i}^{\prime}, g_{i}\right)$ by

$$
\Psi(\Phi(x, t))=\Phi\left(\varphi_{t}(x), t\right) .
$$

This joins the two fiber structures of $\pi_{i}^{\prime}$ and $g_{i}$. Thus we obtain a circle fibration $\pi^{\prime \prime}: M_{i}^{\prime \prime} \rightarrow X^{\prime \prime}$ gluing the fibrations $\pi_{i}^{\prime}: M_{i}^{\prime} \rightarrow X^{\prime}$ and $g_{i}$, where $X^{\prime \prime}=X-$ $\left(U\left(x_{1}, r / 4\right) \cup \cdots \cup U\left(x_{k}, r / 4\right)\right)$.

Let $V_{\mu, \nu}=S^{1} \times D^{2}$ denote the fibered solid torus of type $(\mu, \nu)$.

From now on, for simplicity, we denote $B\left(g_{i}\right)$ by $B_{i}$, and use the same notation as in (4.2). In particular, we have the $\mathbb{Z}_{2}$-equivariant homeomorphism $\hat{f}_{i}: V_{\mu, \nu} \rightarrow \hat{B}_{i}$. Using $\hat{f}_{i}$, we have a fiber structure on $\partial V_{\mu, \nu}$ induced from the $\hat{g}_{i}$-fibers which is isotopic to the standard fiber structure of type $(\mu, \nu)$. 
Assertion 4.6. $(\mu, \nu)=(1,1)$ and there is a $\hat{\sigma}$-equivariant isotopy of $\partial V_{1,1}$ joining the two fiber structures on $\partial V_{1,1}$.

Proof. First suppose $(\mu, \nu)=(1,1)$. On the torus $\partial V_{1,1}=S^{1} \times \partial D^{2}$, let $m=$ $m(t)=\left(1, e^{i t}\right)$ and $\ell=\ell(t)=\left(e^{i t}, 1\right)$ denote the meridian and the longitude. Fix a meridian $m_{i}$ and a longitude $\ell_{i}$ of $\partial \hat{B}_{i}$ such that each fiber of $\hat{g}_{i}$ transversally meets $m_{i}$. Here we may assume that all the longitudes of $\partial \hat{B}_{i}$ discussed below are $\hat{g}_{i}$-fibers.

Set $h_{i}:=\left(\hat{f}_{i}\right)^{-1}$ for simplicity. We now show that $h_{i}\left(\ell_{i}\right)$ is $\hat{\sigma}$-equivariantly ambient isotopic to $\ell$. Recall that $\hat{\pi}: \partial V_{1,1}=S^{1} \times \partial D^{2} \rightarrow K^{2}=S^{1} \times \partial D^{2} / \hat{\sigma}$ is the projection. Since $h_{i}\left(\ell_{i}\right)$ is homotopic to $\ell, \hat{\pi}\left(h_{i}\left(\ell_{i}\right)\right)$ is homotopic to $\hat{\pi}(\ell)$, and hence is ambient isotopic to $\hat{\pi}(\ell)$. Namely, there exists an isotopy $\varphi_{t}, 0 \leq t \leq 1$, of $K^{2}$ such that

$$
\varphi_{0}=i d, \quad \varphi_{1}\left(\hat{\pi}\left(h_{i}\left(\ell_{i}\right)\right)\right)=\hat{\pi}(\ell) .
$$

Let $\hat{\varphi}_{t}: \partial V_{1,1} \rightarrow \partial V_{1,1}$ be the lift of $\varphi_{t}$ such that $\hat{\varphi}_{0}=i d$. Note that $\hat{\varphi}_{1}\left(h_{i}\left(\ell_{i}\right)\right)=\ell$. Therefore we may assume that $h_{i}\left(\ell_{i}\right)=\ell$ from the beginning.

Next we claim that $h_{i}\left(m_{i}\right)$ is $\hat{\sigma}$-equivariantly ambient isotopic to $m$ while keeping $\ell$ fixed. Namely, we show that there exists an isotopy $\hat{\varphi}_{t}, 0 \leq t \leq 1$, of $\partial V_{1,1}$ such that

$$
\hat{\varphi}_{0}=i d, \quad \hat{\varphi}_{1}\left(h_{i}\left(m_{i}\right)\right)=m,\left.\quad \hat{\varphi}\right|_{\ell}=1_{\ell} .
$$

To show this, we proceed in a way similar to the above. Since $h_{i}\left(m_{i}\right)$ is homotopic to $m, \hat{\pi}\left(h_{i}\left(m_{i}\right)\right)$ is homotopic to $\hat{\pi}(m)$, and hence is ambient isotopic to $\hat{\pi}(m)$. Here the construction of isotopy is local (see [E]). Hence approximating $m$ near the intersection point $\ell \cap m$ via a PL-arc for instance, we can choose such an isotopy $\varphi_{t}, 0 \leq t \leq 1$, of $K^{2}$ such that

$$
\varphi_{0}=i d, \varphi_{1}\left(\hat{\pi}\left(h_{i}\left(m_{i}\right)\right)\right)=\hat{\pi}(m),\left.\varphi_{t}\right|_{\hat{\pi}(\ell)}=1_{\hat{\pi}(\ell)} .
$$

Let $\hat{\varphi}_{t}: \partial V_{1,1} \rightarrow \partial V_{1,1}$ be the lift of $\varphi_{t}$ such that $\hat{\varphi}_{0}=i d$. Note that $\hat{\varphi}_{1}$ sends $h_{i}\left(m_{i}\right)$ to $m$ and $\hat{\varphi}_{t}$ is the required isotopy. Therefore we may assume that $h_{i}\left(m_{i}\right)=m$ from the beginning.

For a small $\epsilon>0$, let $\ell^{\prime}=\left(e^{i t}, e^{i \epsilon}\right)$ and $\ell^{\prime \prime}=\left(e^{i t}, e^{-i \epsilon}\right)\left(\right.$ resp. $m^{\prime}=\left(e^{i \epsilon}, e^{i t}\right)$ and $\left.m^{\prime \prime}=\left(e^{-i \epsilon}, e^{i t}\right)\right)$ be longitudes near $\ell$ (resp. meridians near $\left.m\right)$. Let $\ell_{i}^{\prime}$ and $\ell_{i}^{\prime \prime}$ (resp. $m_{i}^{\prime}$ and $m_{i}^{\prime \prime}$ ) be longitudes (resp. meridians) near $\ell_{i}$ (resp. near $m_{i}$ ) such that $\ell_{i}^{\prime}, \ell_{i}^{\prime \prime}, m_{i}^{\prime}$ and $m_{i}^{\prime \prime}$ bound a regular neighborhood of $\ell_{i} \cup m_{i}$. In a way similar to the above, taking a $\hat{\sigma}$-equivariant ambient isotopy, we may assume that $h_{i}\left(\ell_{i}^{\prime}\right)=\ell^{\prime}$, $h_{i}\left(\ell_{i}^{\prime \prime}\right)=\ell^{\prime \prime}, h_{i}\left(m_{i}^{\prime}\right)=m^{\prime}$ and $h_{i}\left(m_{i}^{\prime \prime}\right)=m^{\prime \prime}$.

Let $D$ (resp. $D_{i}$ ) be the small domain bounded by $\ell, \ell^{\prime}, m$ and $m^{\prime}$ (resp. $\ell_{i}$, $\ell_{i}^{\prime}, m_{i}$ and $\left.m_{i}^{\prime}\right)$. Identify $D=I_{0} \times[0,1], D_{i}=I_{i} \times[0,1]$, where $I_{0} \subset m, I_{i} \subset m_{i}$ are arcs, and define $k_{i}: D \rightarrow D$ by $k_{i}(x, t)=h_{i}\left(\hat{f}_{i}(x), t\right)$. From what we have discussed above, $\left.k_{i}\right|_{\partial D}=1_{\partial D}$. It is then standard to obtain an isotopy $\psi_{t}$ of $D$ which sends $k_{i}$ to $1_{D}$ keeping $\partial D$ fixed. Extending $\psi_{t} \hat{\sigma}$-equivariantly, we obtain a $\hat{\sigma}$-equivariant isotopy of $\partial V_{1,1}$ which sends the $h_{i}$-image of $I$-fibers of $D_{i}$ to $I$-fibers of $D$ keeping the outside $D$ fixed. Applying this argument to the other domains bounded by longitudes $\ell^{\prime}, \ell^{\prime \prime}, \hat{\sigma}\left(\ell^{\prime}\right), \hat{\sigma}\left(\ell^{\prime \prime}\right)$ and meridians $m^{\prime}, m^{\prime \prime}$ of $\partial V_{1,1}$, we finally construct a $\hat{\sigma}$-equivariant ambient isotopy $\varphi_{t}$ of $\partial V_{1,1}$ sending the $h_{i}$-images of the $\hat{g}_{i}$-fibers in $\partial B_{i}$ to the corresponding longitudes of $\partial V_{1,1}$.

Finally we show that the case $(\mu, \nu)=(2,1)$ never happens. Let us fix a $g_{i}$-fiber, say $k_{i}$, and a standard $(2,1)$-fiber, say $k$, on the fibered torus $\partial V_{2,1}$ of type $(2,1)$. Since $h_{i}\left(k_{i}\right)$ is homotopic to $k$ in $T^{2}$, in a way similar to the above discussion, 
we have a $\hat{\sigma}$-equivariant ambient isotopy $\hat{\varphi}_{t}$ of $\partial V_{2,1}$ such that $\hat{\varphi}_{0}=i d$ and $\hat{\varphi}_{1}$ sends $h_{i}\left(k_{i}\right)$ to $k$. In $S^{1} \times \partial D^{2}, k$ is described as $k(t)=\left(e^{2 i t}, e^{i t}\right)$, and hence $\hat{\sigma} \circ k(t)=\left(e^{-2 i t}, e^{i(t+\pi)}\right)$. Therefore the images $\operatorname{Im}(\hat{\sigma} \circ k), \operatorname{Im}(k)$ of $\hat{\sigma} \circ k$ and $k$ respectively must meet at $\hat{\sigma} \circ k(-\pi)=k(2 \pi)$. On the other hand,

$$
\hat{\sigma} \circ k=\hat{\sigma} \circ \hat{\varphi}_{1}\left(h_{i}\left(k_{i}\right)\right)=\hat{\varphi}_{1} \circ \hat{\sigma}\left(h_{i}\left(k_{i}\right)\right), k=\hat{\varphi}_{1}\left(h_{i}\left(k_{i}\right)\right) .
$$

It turns out that $\operatorname{Im}\left(\hat{\sigma}\left(h_{i}\left(k_{i}\right)\right)\right)=\operatorname{Im}\left(h_{i}\left(\hat{\sigma}_{i}\left(k_{i}\right)\right)\right)$ meets $\operatorname{Im}\left(h_{i}\left(k_{i}\right)\right)$. This implies that $\operatorname{Im}\left(\hat{\sigma}_{i}\left(k_{i}\right)\right)$ meets $\operatorname{Im}\left(k_{i}\right)$, a contradiction to the fact that $g_{i}$ is a $\mathbb{Z}_{2}$-equivariant fibration.

This completes the proof of the assertion.

Obviously the standard fiber structure on $\partial V_{1,1}$ extends to a standard $\hat{\sigma}$-invariant fiber structure on $V_{1,1}$. Now it becomes easy to extend the fiber structure defined by $\hat{g}_{i}$-fibers on $\hat{\partial} B_{i}$ to a $\hat{\sigma}_{i}$-equivariant fiber structure on $\hat{B}_{i}$ of type $(1,1)$ via $h_{i}$, which projects down to a generalized Seifert bundle structure on $B_{i}$ and on $M_{i}$ for large $i$ which is compatible to the fiber structure of $\pi_{i}^{\prime}$. This completes the proof of Lemma 4.5.

This completes the proof of Theorem 1.3 .

\section{The CASE That $\operatorname{dim} X=2$ AND $\partial X \neq \emptyset$}

Let $\left\{M_{i} \mid i=1,2, \ldots\right\}$ be a sequence of three-dimensional closed Alexandrov spaces with curvature $\geq-1$ having a uniform diameter bound. Suppose that $M_{i}$ converges to an Alexandrov surface $X$ with non-empty boundary.

In this section, we provide decompositions of $X$ into $X^{\prime} \cup X^{\prime \prime}$ and of $M_{i}$ into $M_{i}^{\prime} \cup M_{i}^{\prime \prime}$ such that $M_{i}^{\prime}$ fibers over $X^{\prime}$ in the sense of a generalized Seifert fiber space and $M_{i}^{\prime \prime}$ is the closure of the complement of $M_{i}^{\prime}$. We will prove that each component of $M_{i}^{\prime \prime}$ has the structure of a generalized solid torus or a generalized solid Klein bottle, and the circle fiber structure on its boundary is compatible to the circle fiber structure induced by the generalized Seifert fibration.

From now on, we denote by $C$ one of the components of $\partial X$. Since a twodimensional Alexandrov space is a manifold, $C$ is homeomorphic to a circle. Let us fix a small positive number $\varepsilon$. To construct the desired decompositions of $X$ and $M_{i}$, we define a notion of an $\varepsilon$-regular covering of $C$.

Definition 5.1. Let $\left\{B_{\alpha}, D_{\alpha}\right\}_{1 \leq \alpha \leq n}$ be a covering of $C$ by closed subsets in $X$. We say that $\left\{B_{\alpha}, D_{\alpha}\right\}_{1 \leq \alpha \leq n}$ is $\varepsilon$-regular if it satisfies the following:

(1) $\bigcup_{1<\alpha<n} B_{\alpha} \cup D_{\alpha}-C$ is $(2, \varepsilon)$-strained.

(2) Each $B_{\alpha}$ is the closed metric ball $B_{\alpha}=B\left(p_{\alpha}, r_{\alpha}\right)$ centered at $p_{\alpha}$ with radius $r_{\alpha}>0$ such that

$$
\begin{aligned}
& \left|\nabla d_{p_{\alpha}}\right|>1-\varepsilon \text { on } B\left(p_{\alpha}, 2 r_{\alpha}\right)-\left\{p_{\alpha}\right\}, \\
& B_{\alpha} \cap B_{\alpha^{\prime}}=\emptyset \text { for all } \alpha \neq \alpha^{\prime} .
\end{aligned}
$$

Also, the sequence $p_{1}, p_{2}, \ldots, p_{n}$ is consecutive in $C$.

(3) $D_{\alpha}$ forms

$$
D_{\alpha}:=B\left(\gamma_{\alpha}, \delta\right)-\operatorname{int}\left(B_{\alpha} \cup B_{\alpha+1}\right),
$$

where $\gamma_{\alpha}:=\widehat{p_{\alpha} p_{\alpha+1}}$ with $p_{n+1}:=p_{1}$. Here, $\delta>0$ is a small positive number with $\delta \ll \min _{\alpha} r_{\alpha}$. 
(4) For any $x \in D_{\alpha}$, we have

$$
\tilde{\angle} p_{\alpha} x p_{\alpha+1}>\pi-\varepsilon .
$$

For $x \in D_{\alpha}-C$ and $y \in C$ with $|x y|=|x C|$, we have

$$
\begin{aligned}
& \left|\nabla d_{C}\right|(x)>1-\varepsilon, \\
& \left|\tilde{\angle} p_{\alpha} x y-\pi / 2\right|<\varepsilon, \text { and } \\
& \left|\tilde{\angle} p_{\alpha+1} x y-\pi / 2\right|<\varepsilon .
\end{aligned}
$$

The existence of an $\varepsilon$-regular covering of $C$ will be proved in Section 9. We fix an $\varepsilon$-regular covering

$$
\left\{B_{\alpha}, D_{\alpha} \mid \alpha=1,2, \ldots, n\right\}
$$

of $C$.

We consider a closed neighborhood $X_{C}^{\prime \prime}$ of $C$ defined as

$$
X_{C}^{\prime \prime}:=\bigcup_{\alpha=1}^{n} B_{\alpha} \cup D_{\alpha} .
$$

We set

$$
X^{\prime \prime}:=\bigcup X_{C}^{\prime \prime} \text { and } X^{\prime}:=\text { the closure of } X-X^{\prime \prime} .
$$

This is our decomposition $X=X^{\prime} \cup X^{\prime \prime}$.

Since int $X^{\prime}$ has all interior $(2, \varepsilon)$-singular points of $X$, by Theorem 1.3 we obtain a generalized Seifert fibration

$$
\pi_{i}^{\prime}: M_{i}^{\prime} \rightarrow X^{\prime}
$$

for some closed domain $M_{i}^{\prime} \subset M_{i}$. Let us denote by $X^{\text {reg }}$ the complement of a small neighborhood of the union of $\partial X$ and the set of all interior $(2, \varepsilon)$-singular points in $X$. By Theorem 2.25, we may assume that $\pi_{i}^{\prime}$ is both a circle fibration and a $\theta(\varepsilon)$-Lipschitz submersion on $X^{\text {reg }}$. Recall that $\pi_{i}^{\prime-1}\left(X^{\text {reg }}\right)$ is $(3, \theta(\varepsilon))$-regular, for large $i$.

We set $M_{i}^{\prime \prime}:=M_{i}-\operatorname{int} M_{i}^{\prime}$. We will determine the topology of $M_{i}^{\prime \prime}$ in the following subsections.

\subsection{Decomposition of $M_{i}^{\prime \prime}$.}

Let us denote by $M_{i}^{\text {reg }}$ a $(3, \theta(\varepsilon))$-regular closed domain of $M_{i}$ which contains $\pi_{i}^{\prime-1}\left(X^{\mathrm{reg}}\right)$. By Theorem 2.46, we obtain a smooth approximation

$$
f_{i}: U\left(M_{i}^{\mathrm{reg}}\right) \rightarrow N\left(M_{i}^{\mathrm{reg}}\right)
$$

for a neighborhood $U\left(M_{i}^{\text {reg }}\right)$ of $M_{i}^{\text {reg }}$ and some Riemannian manifold $N\left(M_{i}^{\text {reg }}\right)$.

Let us take $p_{\alpha, i} \in M_{i}$ converging to $p_{\alpha} \in C \subset \partial X$, and $\gamma_{\alpha, i}$ a simple arc joining $p_{\alpha, i}$ and $p_{\alpha+1, i}$ converging to $\gamma_{\alpha}$. By the definition of regular covering, we may assume that

is $(3, \theta(\varepsilon))$-regular.

$$
A\left(\bigcup_{\alpha=1}^{N} \gamma_{\alpha, i} ; \delta / 100,10 \max r_{\alpha}\right)
$$

From now on, we fix any index $\alpha \in\{1, \ldots, N\}$ and use the following notation: $p:=p_{\alpha}, p^{\prime}:=p_{\alpha+1}, B:=B_{\alpha}, B^{\prime}:=B_{\alpha+1}, \gamma:=\gamma_{\alpha}$ and $\gamma^{\prime \prime}:=\gamma_{\alpha-1} ;$ and $p_{i}:=p_{\alpha, i}$, $p_{i}^{\prime}:=p_{\alpha+1, i}, \gamma_{i}:=\gamma_{\alpha, i}$ and $\gamma_{i}^{\prime \prime}:=\gamma_{\alpha-1, i}$. To avoid a disordered notation, we assume that all $r_{\alpha}$ are equal to each other, and set $r:=r_{\alpha}$. 
Let $\delta^{\prime}$ be a small positive number with $\delta^{\prime} \ll \delta$. We will construct an isotopy of $B\left(p_{i}, r+\delta^{\prime}\right)$ which deforms the metric ball $B\left(p_{i}, r-\delta^{\prime}\right)$ to some domain $B_{i}$ such that

$$
\begin{aligned}
B_{i} & \approx B\left(p_{i}, r\right) ; \\
\partial B_{i}-U\left(\gamma_{i} \cup \gamma_{i}^{\prime \prime}, 3 \delta / 2\right) & =\pi_{i}^{\prime-1}\left(\partial B(p, r)-U\left(\gamma \cup \gamma^{\prime \prime}, 3 \delta / 2\right)\right) ; \\
\partial B_{i} \cap B\left(\gamma_{i} \cup \gamma_{i}^{\prime \prime}, \delta\right) & =\partial B\left(p_{i}, r-\delta^{\prime}\right) \cap B\left(\gamma_{i} \cup \gamma_{i}^{\prime \prime}, \delta\right) .
\end{aligned}
$$

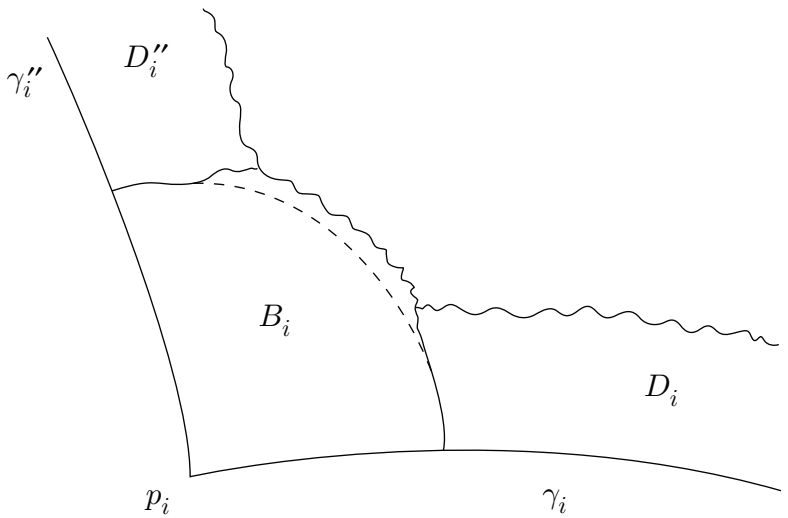

Figure 1. A domain near the corner

In Figure 1, the broken line denotes the metric sphere $S\left(p_{i}, r-\delta^{\prime}\right)$, and the wavy line denotes the pull-back of metric levels with respect to $\gamma^{\prime \prime}, \gamma$ and $p$ in $X$ by $\pi_{i}^{\prime}$.

Suppose that we construct such an isotopy and obtain a domain $B_{i}=B_{\alpha, i}$ satisfying (5.5) and (5.6) for a moment. We consider the domain

$$
D_{i}=D_{\alpha, i}:=B\left(\gamma_{\alpha, i}, 3 \delta / 2\right) \cup \pi_{i}^{\prime-1}\left(A\left(\gamma_{\alpha} ; \delta, 2 \delta\right)\right)-\operatorname{int}\left(B_{\alpha, i} \cup B_{\alpha+1, i}\right) .
$$

Then we obtain a decomposition of $M_{i}^{\prime \prime}$ :

$$
\begin{aligned}
M_{i, C}^{\prime \prime} & :=\bigcup_{\alpha=1}^{N} B_{\alpha, i} \cup D_{\alpha, i}, \\
M_{i}^{\prime \prime} & =\bigcup_{C \subset \partial X} M_{i, C}^{\prime \prime} .
\end{aligned}
$$

Now we construct an isotopy which deforms $B\left(p_{i}, r-\delta^{\prime}\right)$ to $B_{i}$ satisfying (5.5) and (5.6). From now on throughout this paper, we use the following notation. For any set $A \subset M_{i}^{\text {reg }}$, we set $\tilde{A}:=f_{i}(A)$. We denote by $U(A)$ a neighborhood of $A$ in $U\left(M_{i}^{\mathrm{reg}}\right)$ and by $N(A)$ the image of $U(A)$ by the approximation $f_{i}$. Namely, $N(A)=\widetilde{U(A)}$. For any point $x \in A$, we set $\tilde{x}:=f_{i}(x) \in \tilde{A}$. For any function $\phi: A \rightarrow \mathbb{R}$, we define $\tilde{\phi}: \tilde{A} \rightarrow \mathbb{R}$ by

$$
\tilde{\phi}:=\phi \circ f_{i}^{-1} .
$$

Let $\tilde{V}$ be a gradient-like smooth vector field for a Lipschitz function $\widetilde{\operatorname{dist}}_{p_{i}}$ on $N\left(\left(B\left(p_{i}, r\right) \cup B\left(p_{i}^{\prime}, r\right) \cup B\left(\gamma_{i}, 2 \delta\right)\right) \cap M_{i}^{\text {reg }}\right)$ obtained by Lemma 3.7. 
We take a Lipschitz function $h$ defined on $B\left(p_{i}, r+\delta^{\prime}\right)$ such that

$$
\begin{aligned}
& \tilde{h} \text { is smooth, } \\
& 0 \leq h \leq 1, \\
& \operatorname{supp}(h) \subset B\left(p_{i}, r+\delta^{\prime}\right)-U\left(\gamma_{i} \cup \gamma_{i}^{\prime \prime}, \delta / 2\right), \\
& h \equiv 1 \text { on } B\left(p_{i}, r+\delta^{\prime}\right)-U\left(\gamma_{i} \cup \gamma_{i}^{\prime \prime}, \delta\right) .
\end{aligned}
$$

We consider a smooth vector field $\tilde{h} \cdot \tilde{V}$ and its integral flow $\tilde{\Phi}$, and we define the pull-back flow $\Phi_{t}:=f_{i}^{-1} \circ \tilde{\Phi}_{t} \circ f_{i}$. Then by construction and Theorem 3.11, the flow $\Phi$ transversally intersects $\pi_{i}^{\prime-1}\left(\partial B(p, r)-U\left(\gamma \cup \gamma^{\prime \prime}, \delta\right)\right)$. Then we can construct an isotopy by using the flow $\Phi$, which provides a closed neighborhood $B_{i}$ of $p_{i}$ satisfying (5.4), (5.5) and (5.6).

5.2. The topologies of the balls near corners. We first prove that $\partial B_{i}$ is homeomorphic to a closed 2-manifold.

Lemma 5.2. $\partial B_{i} \approx \partial B\left(p_{i}, r\right)$ is a closed 2-manifold.

Proof. If $B_{i}$ does not satisfy Assumption 2.28, we have some sequence $\hat{p}_{i}$ with $\left|\hat{p}_{i} p_{i}\right| \rightarrow 0$ such that $\partial B\left(\hat{p}_{i}, r\right) \approx \Sigma_{\hat{p}_{i}}$, where we may assume that $\hat{p}_{i}=p_{i}$. Since $M_{i}$ has no boundary, $\partial B\left(p_{i}, r\right)$ is homeomorphic to $S^{2}$ or $P^{2}$.

If $B_{i}$ satisfies Assumption 2.28, there exist a sequence $\delta_{i} \rightarrow 0$ and $\hat{p}_{i}$ with $\left|\hat{p}_{i} p_{i}\right| \rightarrow$ 0 such that the limit $\left(Y, y_{0}\right)$ of $\left(\frac{1}{\delta_{i}} B\left(\hat{p}_{i}, r\right), \hat{p}_{i}\right)$ has dimension three. Here, we may assume that $\hat{p}_{i}=p_{i}$. Then, by Soul Theorem 2.59 and Stability Theorem 2.34, $\partial B\left(p_{i}, r\right)$ is homeomorphic to $S^{2}, P^{2}, T^{2}$ or $K^{2}$.

From (5.5) and the construction of $B_{i}$, we have

$$
\partial B_{i}-U\left(\gamma_{i} \cup \gamma_{i}^{\prime \prime}, \delta\right) \approx S^{1} \times I .
$$

Now, we put $F_{i}$ and $F_{i}^{\prime \prime}$ as follows:

$$
F_{i}:=\partial B_{i} \cap B\left(\gamma_{i}, \delta\right) \text { and } F_{i}^{\prime \prime}:=\partial B_{i} \cap B\left(\gamma_{i}^{\prime \prime}, \delta\right) .
$$

Then, by Lemma $5.2, F_{i}$ and $F_{i}^{\prime \prime}$ are 2-manifolds with boundaries homeomorphic to $S^{1}$. By the generalized Margulis lemma [FY], $F_{i}$ has an almost nilpotent fundamental group. Hence $F_{i}$ is homeomorphic to $D^{2}$ or Mö.

Therefore, we obtain the following assertion:

Lemma 5.3. $\partial B_{i}$ is homeomorphic to $S^{2}, P^{2}$ or $K^{2}$.

We now determine the topology of $B_{i}$.

Lemma 5.4. $B_{i}$ is homeomorphic to $D^{3}$, Mö $\times I$ or $K_{1}\left(P^{2}\right)$. Moreover, if $\operatorname{diam} \Sigma_{p}>$ $\pi / 2$, then $B_{i}$ is not homeomorphic to $K_{1}\left(P^{2}\right)$.

Proof. We first consider the case that diam $\Sigma_{p}>\pi / 2$. Then by Proposition 2.70, $\Sigma_{p_{i}}$ is topologically a suspension over a one-dimensional Alexandrov space $\Lambda$ of curvature $\geq 1$. Since $\partial \Sigma_{p_{i}}=\emptyset, \Lambda$ is a circle. Hence $p_{i}$ is a topologically regular point. Note that, in this situation, any $x \in B(p, r)$ has diam $\Sigma_{x}>\pi / 2$. Therefore, $\operatorname{int} B_{i}$ is topologically a manifold, and $B_{i}$ is not homeomorphic to $K_{1}\left(P^{2}\right)$.

From now on we assume that diam $\Sigma_{p} \leq \pi / 2$. If $B_{i}$ does not satisfy Assumption 2.28, then there exists $\hat{p}_{i}$ such that $\lim \left|p_{i} \hat{p}_{i}\right|=0$ and $B\left(\hat{p}_{i}, r\right) \approx K_{1}\left(\Sigma_{\hat{p}_{i}}\right)$ which is homeomorphic to $D^{3}$ or $K_{1}\left(P^{2}\right)$, where we may assume that $p_{i}=\hat{p}_{i}$. 
Suppose that $B_{i}$ satisfies Assumption 2.28. By Theorem 2.27, there is a sequence $\delta_{i}$ of positive numbers tending to zero and points $\hat{p}_{i}$ (where we may assume that $\left.\hat{p}_{i}=p_{i}\right)$ such that

- any limit $\left(Y, y_{0}\right)$ of $\left(\frac{1}{\delta_{i}} B_{i}, p_{i}\right)$ as $i \rightarrow \infty$ is a three-dimensional open Alexandrov space of non-negative curvature;

- denoting by $S$ a soul of $Y$, we obtain $\operatorname{dim} S \leq 1$.

Then, by Soul Theorem 2.59, $Y$ is homeomorphic to $\mathbb{R}^{3}, K\left(P^{2}\right)$ or $M_{\mathrm{pt}}$ if $\operatorname{dim} S=0$ or an $\mathbb{R}^{2}$-bundle over $S^{1}$ if $\operatorname{dim} S=1$. Therefore, $B_{i}$ is homeomorphic to $D^{3}$, $K_{1}\left(P^{2}\right)$ or $B(\mathrm{pt})$ if $\operatorname{dim} S=0$ or $S^{1} \times D^{2}$ or $S^{1} \tilde{\times} D^{2} \approx \mathrm{Mö} \times I$ if $\operatorname{dim} S=1$. By the boundary condition (Lemma 5.3),$B_{i}$ is actually not homeomorphic to $S^{1} \times D^{2}$. It remains to show that

$$
B_{i} \text { is not homeomorphic to } B(\mathrm{pt}) \text {. }
$$

We prove (5.13) by contradiction. Suppose that there is a homeomorphism $f_{i}: B(\mathrm{pt}) \rightarrow B_{i}$. We will use the notation in the proof of Lemma 4.5. Recall that $B(\mathrm{pt})$ is obtained by the quotient space of $S^{1} \times D^{2}$ by the involution $\hat{\sigma}$. We consider the corresponding space $\hat{B}_{i}$ with an involution $\hat{\sigma}_{i}$ such that its quotient is $B_{i}$. By the argument of the proof of Lemma 4.5, we obtain the following commutating diagram:

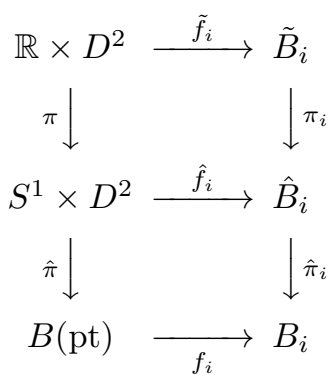

Here, the horizontal arrows are homeomorphisms, $\pi$ and $\pi_{i}$ are the universal coverings, and $\hat{\pi}$ and $\hat{\pi}_{i}$ are the projections by involutions $\hat{\sigma}$ and $\hat{\sigma}_{i}$, respectively. We may assume that $\left(\tilde{B}_{i}, \tilde{p}_{i}, \Gamma_{i}, \Lambda_{i}\right)$ converges to $\left(Z, z_{0}, \Gamma_{\infty}, \Lambda_{\infty}\right)$ with $Z=\mathbb{R} \times L$, $\Lambda_{\infty}=\mathbb{R} \times H, L$ is a flat cone over a circle and $H$ is a finite abelian group acting on $L$. Note that all elements of $H$ are orientation preserving on $L$. Recall that $\sigma_{\infty}$ is expressed as $\sigma_{\infty}(x, y)=\left(-x, \sigma_{\infty}^{\prime}(y)\right)$ and $\sigma_{\infty}$ is orientation preserving on $L$. Therefore, $\left[\sigma_{\infty}^{\prime}\right]$ is orientation preserving on $L / H$. We remark that $(L / H) /\left[\sigma_{\infty}^{\prime}\right]=T_{p} X$. Then, $L / H$ has no boundary. Indeed, to check this, we suppose that $L / H$ has nonempty boundary. Then $L / H$ is the cone over an arc. Since $\left[\sigma_{\infty}^{\prime}\right]$ is a non-trivial isometry on $L / H,\left[\sigma_{\infty}^{\prime}\right]$ is the reflection with respect to the center line. Therefore, $\left[\sigma_{\infty}^{\prime}\right]$ does not preserve orientation. This is a contradiction.

Thus, $L / H$ is the cone over a circle. It turns out that $\sigma_{\infty}^{\prime}$ is a half rotation of $L$, and hence so is $\left[\sigma_{\infty}^{\prime}\right]$ for $L / H$. This implies $T_{p} X$ has no boundary, and we obtain a contradiction. We conclude (5.13), and complete the proof of Lemma 5.4

Next, we will divide $D_{i}$ into two pieces $D_{i}=H_{i} \cup K_{i}$ depending on the topology of $F_{i}$. We will also determine the topology of $H_{i}, K_{i}$, and $D_{i}$. 
5.3. The case that $F_{i}$ is a disk. We consider the case that $F_{i} \approx D^{2}$. Then, we divide $D_{i}$ into $H_{i}$ and $K_{i}$ as follows:

$$
\begin{aligned}
& H_{i}:=D_{i}-U\left(\gamma_{i}, \delta\right), \\
& K_{i}:=D_{i} \cap B\left(\gamma_{i}, \delta\right) .
\end{aligned}
$$

5.3.1. The topology of $K_{i}$. We prove that

Assertion 5.5. $K_{i}$ is homeomorphic to $D^{3}$.

$K_{i}$ is contained in a domain $L_{i}$ defined by

$$
L_{i}:=A\left(p_{i} ; r-\delta^{\prime},\left|p p^{\prime}\right|-r / 2\right) \cap B\left(\gamma_{i}, \delta\right) .
$$

Since $\left(d_{p_{i}}, d_{\gamma_{i}}\right)$ is $(c, \theta(\varepsilon))$-regular near $L_{i} \cap S\left(\gamma_{i}, \delta\right)$, by Theorem 2.33 and Lemma 2.40, $L_{i}$ is homeomorphic to $F_{i} \times[0,1] \approx D^{3}$. On the other hand, we can take a closed domain $A_{i} \subset \operatorname{int} K_{i}$ such that $A_{i} \approx D^{3}$ and

$$
K_{i}^{0}:=B\left(\gamma_{i}, \delta / 2\right)-\left(U\left(p_{i}, 2 r\right) \cup U\left(p_{i}^{\prime}, 2 r\right)\right) \subset \operatorname{int} A_{i} .
$$

By Theorem 2.33 and Lemma 2.40, $K_{i} \approx K_{i}^{0}$. Remark that $F_{i}^{\prime}:=\partial B_{i}^{\prime} \cap B\left(\gamma_{i}, \delta\right)$ is homeomorphic to $D^{2}$. Indeed, if we assume that $F_{i}^{\prime} \approx \mathrm{Mö}$, then $\partial K_{i} \approx P^{2}$. Then, by the embedding (5.15), we have

$$
P^{2} \approx \partial K_{i}^{0} \subset \operatorname{int} A_{i} \approx \mathbb{R}^{3} .
$$

This is a contradiction. Therefore, $F_{i}^{\prime} \approx D^{2}$ and $\partial K_{i}^{0} \approx \partial K_{i} \approx S^{2}$. By Theorem 2.33. $\partial K_{i}^{0}$ is locally flatly embedded in $A_{i} \approx D^{3}$. Therefore, by the generalized Schoenflies theorem, we conclude $K_{i} \approx K_{i}^{0} \approx D^{3}$.

5.3.2. The topology of $H_{i}$.

Assertion 5.6. $H_{i}$ is homeomorphic to $S^{1} \times D^{2}$ and the circle fiber structure on $H_{i}$ induced by the standard one on $S^{1} \times D^{2}$ is compatible to $\pi_{i}^{\prime}$.

Let us define a domain $Q \subset X$ by

$$
Q:=A\left(\gamma ; \delta-\delta^{\prime}, 2 \delta+\delta^{\prime}\right)-\left(U\left(p, r-2 \delta^{\prime}\right) \cup U\left(p^{\prime}, r-2 \delta^{\prime}\right)\right) .
$$

Note that $Q$ is homeomorphic to a two-disk without $(2, \varepsilon)$-singular points. Then $Q_{i}:=\pi_{i}^{\prime-1}(Q)$ is topologically a solid torus, and $H_{i}$ is contained in the interior of $Q_{i}$.

We will construct an isotopy $\varphi: Q_{i} \times[0,1] \rightarrow Q_{i}$ satisfying

$$
\begin{aligned}
& \varphi(\cdot, 0)=\operatorname{id}_{Q_{i}}, \\
& \varphi\left(Q_{i}, 1\right)=H_{i}, \\
& \varphi: \partial Q_{i} \times[0,1] \rightarrow Q_{i}-\operatorname{int} H_{i} \text { is bijective. }
\end{aligned}
$$

If we obtain such a $\varphi$, then by (5.18), we conclude $H_{i} \approx Q_{i} \approx S^{1} \times D^{2}$. And by (5.19), we can obtain the circle fiber structure of $H_{i}$ over $Q$ which is compatible to the generalized Seifert fibration $\pi_{i}^{\prime}$.

Next we use the conventions as in (5.10).

Lemma 5.7. There is a smooth vector field $\tilde{X}$ on $N\left(Q_{i}-H_{i}\right)$ such that it is gradient-like:

- for $\tilde{d}_{p_{i}}$ and $\widetilde{d_{p} \circ \pi_{i}^{\prime}}$ on $N\left(B\left(p_{i}, r+\delta^{\prime}\right) \cap Q_{i}-H_{i}\right)$,

- for $\widetilde{d}_{p_{i}^{\prime}}$ and $\widetilde{d_{p^{\prime}} \circ \pi_{i}^{\prime}}$ on $N\left(B\left(p_{i}^{\prime}, r+\delta^{\prime}\right) \cap Q_{i}-H_{i}\right)$, 
- for $\widetilde{d}_{\gamma_{i}}$ and $\widetilde{d_{\gamma} \circ \pi_{i}^{\prime}}$ on $N\left(B\left(\gamma_{i}, \delta+\delta^{\prime}\right) \cap Q_{i}-H_{i}\right)$, and - for $-\widetilde{d}_{\gamma_{i}}$ and $-\widetilde{d_{\gamma} \circ \pi_{i}^{\prime}}$ on $N\left(Q_{i}-H_{i}-U\left(\gamma_{i}, 2 \delta-\delta^{\prime}\right)\right)$.

Proof. Let us take gradient-like smooth vector fields $\tilde{V}, \tilde{V}^{\prime}$ and $\tilde{W}$ for $\widetilde{d}_{p_{i}}, \widetilde{d}_{p_{i}^{\prime}}$ and $\widetilde{d}_{\gamma_{i}}$ on $N\left(Q_{i}-H_{i}\right)$. We prepare a decomposition of $Q_{i}-\operatorname{int} H_{i}$ as follows:

$$
Q_{i}-\operatorname{int} H_{i}=\bigcup_{\alpha=1}^{8} A_{\alpha} .
$$

See Figure 2, Here, we define

$$
\begin{aligned}
& A_{1}:=\left(Q_{i}-\operatorname{int} H_{i}\right) \cap\left(B\left(\gamma_{i}, \delta\right)-U\left(\left\{p_{i}, p_{i}^{\prime}\right\}, r+\delta^{\prime}\right)\right), \\
& A_{2}:=\left(Q_{i}-\operatorname{int} H_{i}\right)-\left(U\left(\gamma_{i}, 2 \delta-\delta^{\prime}\right) \cup U\left(\left\{p_{i}, p_{i}^{\prime}\right\}, r+\delta^{\prime}\right)\right), \\
& A_{3}^{*}:=B\left(p_{i}, r+\delta^{\prime}\right) \cap B\left(\gamma_{i}, \delta+\delta^{\prime}\right), \\
& A_{4}^{*}:=B\left(p_{i}, r+\delta^{\prime}\right) \cap A\left(\gamma_{i} ; \delta+\delta^{\prime}, 2 \delta-\delta^{\prime}\right), \\
& A_{5}^{*}:=B\left(p_{i}, r+\delta^{\prime}\right)-U\left(\gamma_{i}, 2 \delta-\delta^{\prime}\right) .
\end{aligned}
$$

Similarly, we put

$$
\begin{aligned}
& A_{6}^{*}:=B\left(p_{i}^{\prime}, r+\delta^{\prime}\right) \cap B\left(\gamma_{i}, \delta+\delta^{\prime}\right), \\
& A_{7}^{*}:=B\left(p_{i}^{\prime}, r+\delta^{\prime}\right) \cap A\left(\gamma_{i} ; \delta+\delta^{\prime}, 2 \delta-\delta^{\prime}\right), \\
& A_{8}^{*}:=B\left(p_{i}^{\prime}, r+\delta^{\prime}\right)-U\left(\gamma_{i}, 2 \delta-\delta^{\prime}\right) .
\end{aligned}
$$

We define $A_{3}, A_{4}, \cdots, A_{8}$ by

$$
A_{\alpha}:=A_{\alpha}^{*} \cap Q_{i}-\operatorname{int} H_{i} \text { for } \alpha=3,4, \ldots, 8 \text {. }
$$

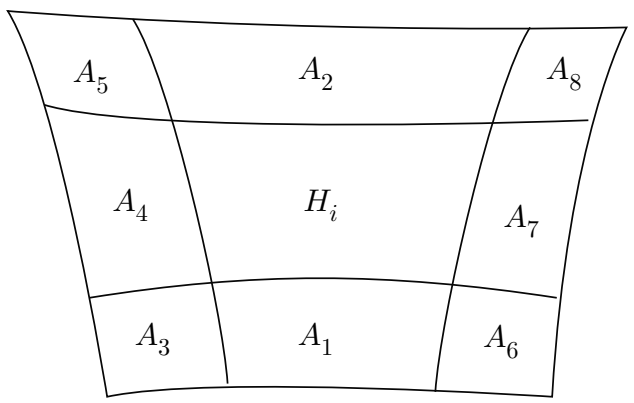

Figure 2. The decomposition of $Q_{i}$

We take smooth functions $h_{\alpha}(\alpha=1, \ldots, 8)$ on $N\left(Q_{i}-H_{i}\right)$ such that

$$
\begin{aligned}
& 0 \leq h_{\alpha} \leq 1, \\
& h_{\alpha} \equiv 1 \text { on } \tilde{A}_{\alpha}, \\
& \operatorname{supp}\left(h_{\alpha}\right) \subset B\left(\tilde{A}_{\alpha}, \delta^{\prime} / 100\right) .
\end{aligned}
$$

We define a vector field $\tilde{X}$ as

$$
\begin{aligned}
\tilde{X}:= & h_{1} \tilde{W}-h_{2} \tilde{W} \\
& +h_{3}(\tilde{V}+\tilde{W})+h_{4} \tilde{V}+h_{5}(\tilde{V}-\tilde{W}) \\
& +h_{6}\left(\tilde{V}^{\prime}+\tilde{W}\right)+h_{7} \tilde{V}^{\prime}+h_{8}\left(\tilde{V}^{\prime}-\tilde{W}\right) .
\end{aligned}
$$


Then, we can show that $\tilde{X}$ satisfies the conclusion of Lemma 5.7 as follows. We will prove it only on $N\left(A_{3}\right)$.

We consider the integral flow $\tilde{\Phi}$ of $\tilde{X}$ and the pull-back $\Phi_{t}:=f_{i}^{-1} \circ \tilde{\Phi}_{t} \circ f_{i}$. It suffices to show that

$$
\begin{aligned}
& \Phi \pitchfork \operatorname{dist}_{p_{i}} \\
& \Phi \pitchfork \operatorname{dist}_{\gamma_{i}} \\
& \Phi \pitchfork \operatorname{dist}_{p} \circ \pi_{i}^{\prime} \\
& \Phi \pitchfork \operatorname{dist}_{\gamma} \circ \pi_{i}^{\prime}
\end{aligned}
$$

on $N\left(A_{3}\right)$. We can write

$$
\tilde{X}=\alpha \tilde{V}+\beta \tilde{W}
$$

for smooth functions $\alpha, \beta \geq 0$ with $1 \leq \alpha+\beta \leq 3$ on $N\left(A_{3}\right)$. By a direct calculus, we have

$$
\begin{gathered}
|\tilde{X}| \geq \sqrt{2}-\theta(\varepsilon), \\
\angle(\tilde{X}, \tilde{V})<\gamma+\theta(\varepsilon), \angle(\tilde{X}, \tilde{W})<\gamma+\theta(\varepsilon)
\end{gathered}
$$

on $N\left(A_{3}\right)$. Here, $\cos \gamma=1 / \sqrt{10}$.

Let us set

$$
X:=\left.\frac{d}{d t}\right|_{t=0+} \Phi(x, t) \in T_{x} M_{i} .
$$

Then we have

$$
X(x)=d f_{i}^{-1}(\tilde{X}(\tilde{x}))
$$

We set

$$
V:=d f_{i}^{-1}(\tilde{V}), W:=d f_{i}^{-1}(\tilde{W}) .
$$

Then we obtain

$$
V \doteqdot \nabla d_{p_{i}}, W \doteqdot \nabla d_{\gamma_{i}}
$$

Here, $A \doteqdot A^{\prime}$ means $\left|A, A^{\prime}\right|<\theta(\varepsilon)$.

Since $f_{i}$ is a $\theta(\varepsilon)$-almost isometry, we have

$$
|X| \doteqdot|\tilde{X}|,|V, X| \doteqdot|\tilde{V}, \tilde{X}|, \angle(V, X) \doteqdot \angle(\tilde{V}, \tilde{X})
$$

Hence, we obtain

$$
\begin{aligned}
|X| & \geq \sqrt{2}-\theta(\varepsilon), \\
\angle\left(p_{i}^{\prime}, X\right) & \geq \angle\left(p_{i}^{\prime}, V\right)-\angle(V, X)>\pi-\gamma-\theta(\varepsilon) .
\end{aligned}
$$

Therefore, we have

$$
\left(d_{p_{i}}\right)^{\prime}(X)=-|X| \cos \angle\left(p_{i}^{\prime}, X\right) \geq 1 / \sqrt{5}-\theta(\varepsilon) .
$$

This implies (5.21).

For any fixed scale $(o)$, we set

$$
d \pi_{i}^{\prime}:=\left(\exp _{\pi_{i}^{\prime}(x)}^{(o)}\right)^{-1} \circ\left(\pi_{i}^{\prime}\right)_{x}^{(o)} \circ \exp _{x}^{(o)} .
$$

By Proposition 3.10, we have

$$
\angle\left(p^{\prime}, d \pi_{i}^{\prime}(X)\right)<\gamma+\theta(\varepsilon),\left|d \pi_{i}^{\prime}(X)\right|>\sqrt{2}-\theta(\varepsilon) .
$$


Therefore, we obtain

$$
\begin{aligned}
\left(\operatorname{dist}_{p} \circ \pi_{i}^{\prime}\right)_{x}^{(o)}\left(\exp _{x}^{(o)}(X)\right) & =-\left|d \pi_{i}^{\prime}(X)\right| \cos \angle_{\pi_{i}^{\prime}(x)}\left(p^{\prime}, d \pi_{i}^{\prime}(X)\right) \\
& >1 / \sqrt{5}-\theta(\varepsilon) .
\end{aligned}
$$

Thus, we obtain (5.23).

In a similar way to the above, we can prove (5.22) and (5.24).

By Lemma 5.7, we obtain an isotopy $\varphi$ based on $\Phi$ satisfying (5.17) through (5.19).

Therefore, we conclude that if $F_{i} \approx D^{2}$, then $D_{i} \approx D^{3}$.

5.4. The case that $F_{i}$ is a Mobius band. We consider the case that $F_{i} \approx$ Mö. We prove that

Lemma 5.8. $D_{i}$ is $(3, \theta(\varepsilon))$-strained.

Proof. We first define a domain $L_{i}$ similar to (5.14):

$$
L_{i}:=A\left(p_{i} ; r / 2,\left|p p^{\prime}\right|-r / 2\right) \cap B\left(\gamma_{i}, 3 \delta\right) .
$$

To prove Lemma 5.8, it suffices to show that

$$
L_{i} \text { is }(3, \theta(\varepsilon)) \text {-regular. }
$$

By Theorem 2.33 and Lemma 2.40, we have $L_{i} \approx \mathrm{Mö} \times I$. Let $\hat{L}_{i}$ be the orientable double cover which is homeomorphic to $\left(S^{1} \times I\right) \times I$. Since $\hat{L}_{i}$ is a covering space of $L_{i}, \hat{L}_{i}$ has the metric of Alexandrov space with $L_{i} \equiv \hat{L}_{i} /\langle\sigma\rangle$ for an isometric involution $\sigma$ on $\hat{L}_{i}$.

Since the projection $\hat{L}_{i} \rightarrow L_{i}$ is a local isometry, to prove (5.25), it suffices to show that

$$
\hat{L}_{i} \text { is }(3, \theta(\varepsilon)) \text {-regular. }
$$

$L_{i}$ converges to the following closed domain $L_{\infty}$ in $X$ :

$$
L_{\infty}=A\left(p ; r / 2,\left|p p^{\prime}\right|-r / 2\right) \cap B(\gamma, 3 \delta) .
$$

We may assume that $\hat{L}_{i}$ converges to some two-dimensional space $Y^{2}$. Note that $L_{\infty}$ is 1-strained, and hence $L_{i}$ and $\hat{L}_{i}$ are also 1-strained. Therefore,

$$
Y \text { is 1-strained. }
$$

From the form of $L_{\infty}$, we have that $Y^{2}$ is a two-disk having no $\varepsilon$-singular points. Indeed, if $Y$ has a boundary-point in the sense of Alexandrov space, then from an argument similar to the proof of Assertion 5.5, $\hat{L}_{i}$ contains a domain homeomorphic to $D^{2} \times I$ or $M \ddot{0} \times I$. This is a contradiction, and hence $Y$ has no boundary. By this and (5.27), $Y$ is 2-strained. Therefore, $\hat{L}_{i}$ is 3 -strained; this is the assertion (5.26). This implies (5.25) and completes the proof of Lemma 5.8.

By Lemma 5.8 and Theorem 3.2, we have a Lipschitz flow $\Phi$ which is gradient-like for dist $p_{i}$ near $D_{i}$. We divide $D_{i}$ into $H_{i}$ and $K_{i}$ as follows:

$$
\begin{aligned}
K_{i}:= & \text { the union of flow curves of } \Phi \\
& \text { starting from } F_{i} \text { in } B\left(\gamma_{i}, 2 \delta\right)-\operatorname{int} B_{i}^{\prime} . \\
H_{i}:= & D_{i}-\operatorname{int} K_{i} .
\end{aligned}
$$


Note that the union of flow curves of $\Phi$ starting from $\partial F_{i}$ is contained in $A\left(\gamma_{i} ; \delta-\delta^{\prime \prime}, \delta+\delta^{\prime \prime}\right)$ for some small $\delta^{\prime \prime}>0$. By the construction, $K_{i} \approx$ Mö $\times I$.

We will prove that

Assertion 5.9. $H_{i}$ is homeomorphic to $S^{1} \times D^{2}$ and the circle fiber structure on $H_{i}$ induced by one on $S^{1} \times D^{2}$ is compatible to $\pi_{i}^{\prime}$.

Proof. Let $Q_{i}$ be a closed neighborhood of $H_{i}$ obtained in a way similar to the construction of $Q_{i}$ in subsection 5.3. We actually define

$$
\begin{aligned}
Q & :=A\left(\gamma ; \delta-\delta^{\prime \prime}, 2 \delta+\delta^{\prime}\right)-U\left(\left\{p, p^{\prime}\right\}, r-2 \delta^{\prime}\right), \\
Q_{i} & :=\pi_{i}^{\prime-1}(Q) .
\end{aligned}
$$

We prepare a decomposition of $Q_{i}-\operatorname{int} H_{i}=\bigcup_{\alpha=1}^{8} A_{\alpha}$ in a way similar to (5.20) in Lemma 5.7. Actually, we define $A_{5}, A_{2}, A_{8}$ as in Lemma 5.7, and other $A_{\alpha}$ 's are defined by

$$
\begin{aligned}
& A_{1}:=\left(Q_{i}-\operatorname{int} H_{i}\right) \cap\left(B\left(\gamma_{i}, \delta+\delta^{\prime \prime}\right)-U\left(\left\{p_{i}, p_{i}^{\prime}\right\}, r+\delta^{\prime}\right)\right), \\
& A_{3}:=\left(Q_{i}-\operatorname{int} H_{i}\right) \cap B\left(p_{i}, r+\delta^{\prime}\right) \cap B\left(\gamma_{i}, \delta+\delta^{\prime \prime}\right), \\
& A_{6}:=\left(Q_{i}-\operatorname{int} H_{i}\right) \cap B\left(p_{i}^{\prime}, r+\delta^{\prime}\right) \cap B\left(\gamma_{i}, \delta+\delta^{\prime \prime}\right), \\
& A_{4}:=\left(Q_{i}-\operatorname{int}\left(H_{i} \cup A_{3} \cup A_{5}\right)\right) \cap B\left(p_{i}, r+\delta^{\prime}\right), \\
& A_{7}:=\left(Q_{i}-\operatorname{int}\left(H_{i} \cup A_{6} \cup A_{8}\right)\right) \cap B\left(p_{i}^{\prime}, r+\delta^{\prime}\right) .
\end{aligned}
$$

Since $\nabla$ dist $_{p_{i}}$ and $\nabla$ dist $_{\gamma_{i}}$ are almost perpendicular to each other on $Q_{i}-\operatorname{int} H_{i}$, we can obtain a flow $\Phi$ which has nice transversality as in Lemma 5.7. We can also construct an isotopy from the identity to some homeomorphism which deforms $Q_{i}$ to $H_{i}$ inside $Q_{i}$. Therefore, we obtain a circle fibration of $H_{i}$ over $Q$ which is compatible to the generalized Seifert fibration $\pi_{i}^{\prime}$. This completes the proof of Assertion 5.9 .

Therefore, we conclude that if $F_{i} \approx \mathrm{Mö}$, then $D_{i} \approx \mathrm{Mö} \times I$.

Proof of Theorem 1.5. It remain to show that each component $M_{i, C}^{\prime \prime}$ of $M_{i}^{\prime \prime}$ has the structure of a generalized solid torus or generalized solid Klein bottles. This is clear from Sections 5.3 and 5.4

5.5. Proof of Corollary 1.6. To prove Corollary 1.6. we show elementary lemmas. We define the mapping class group $\operatorname{MCG}(F)$ of a topological space $F$ to be the set of all isotopy classes of homeomorphisms of $F$.

Lemma 5.10. Let $F$ be a topological space. For any element $\gamma$ of the mapping class group $\operatorname{MCG}(F)$, we fix a homeomorphism $\varphi_{\gamma}: F \rightarrow F$ such that $\varphi_{\gamma} \in \gamma$. Let us set $B=F \times[0,1]$ and $\pi: B \rightarrow[0,1]$ a projection. For any homeomorphisms $f_{i}: F \rightarrow \pi^{-1}(i)$, for $i=0,1$, there exist $\gamma \in \operatorname{MCG}(F)$ and a homeomorphism $h: F \times[0,1] \rightarrow B$ respecting $\pi$ such that, for every $x \in F, h(x, 0)=f_{0}(x)$ and $h(x, 1)=f_{1} \circ \varphi_{\gamma}(x)$.

Proof. Let us set $F_{t}=\pi^{-1}(t)=F \times\{t\}$. Let us define the translation $\chi_{t}: F_{0} \rightarrow F_{t}$ by $\chi_{t}(x, 0)=(x, t)$, and set a homeomorphism $\tilde{f}_{t}=\chi_{t} \circ f_{0}: F \rightarrow F_{t}$. Note that $\tilde{f}_{0}=f_{0}$. Let us take an element $\gamma \in \operatorname{MCG}(F)$ represented by a homeomorphism $f_{1}^{-1} \circ \tilde{f}_{1}$ of $F$. Then, there is a homeomorphism $g_{t}: F \rightarrow F$, for $0 \leq t \leq 1$, such that

$$
g_{0}=\mathrm{id} \text { and } \tilde{f}_{1} \circ g_{1}=f_{1} \circ \varphi_{\gamma} .
$$


Therefore, setting $h_{t}=\tilde{f}_{t} \circ g_{t}: F \rightarrow F_{t}$, we obtain

$$
h_{0}=f_{0} \text { and } h_{1}=f_{1} \circ \varphi_{\gamma} \text {. }
$$

Hence, defining $h: F \times[0,1] \rightarrow B$ by $h(x, t)=h_{t}(x), h$ satisfies the desired condition.

Lemma 5.11. Let $Y$ be a generalized solid torus or a generalized solid Klein bottle. Let $\pi: Y \rightarrow S^{1}$ be a projection as in (1.2). Then, there is a continuous surjection

$$
\eta: Y \rightarrow[0,1]
$$

such that $\eta^{-1}(1)=\partial Y$ and, setting

$$
\Phi=(\pi, \eta): Y \rightarrow S^{1} \times[0,1],
$$

$\Phi$ is an $S^{1}$-bundle over $S^{1} \times(0,1]$. Further, for every $x \in S^{1}, \Phi^{-1}(x, 0)$ is a one point set or a circle, and the homeomorphic type of the fiber $\Phi^{-1}(x, 0)$ changes if and only if that of $\pi^{-1}(x)$ changes.

Proof. Let us take ordered points $t_{1}, t_{2}, \ldots, t_{2 N-1}, t_{2 N} \in S^{1}$ changing the fiber of $\pi$. Then, for a small $\varepsilon>0$, setting $I_{k}=\left[t_{k}-\varepsilon, t_{k}+\varepsilon\right] \subset S^{1}, \pi^{-1}\left(I_{k}\right)$ is homeomorphic to $K_{1}\left(P^{2}\right)$.

We regard $K_{1}\left(P^{2}\right)=\bigcup_{t \in[-1,1]} D(t)$ as in Definition 1.4 Let us define a continuous surjection $\theta: K_{1}\left(P^{2}\right) \rightarrow[0,1]$ by

$$
\theta(x, y, z)= \begin{cases}z^{2} & \text { if } t>0 \\ x^{2}+y^{2} & \text { if } t \leq 0\end{cases}
$$

This is well defined. ( $\theta$ is like the square of the distance function from the center of each surface $D(t)$. If $t \leq 0$, then the center means a point $D(t) \cap\left\{x^{2}+y^{2}=0\right\}$ of disk $D(t)$, and if $t>0$, then the center means a centric circle $D(t) \cap\{z=0\}$ of a Mobius band $D(t)$.) Let us fix a homeomorphism $\varphi_{k}: K_{1}\left(P^{2}\right) \rightarrow \pi^{-1}\left(I_{k}\right)$ respecting $\pi$. We define a continuous surjection

$$
\eta_{k}=\theta \circ \varphi_{k}^{-1}: \pi^{-1}\left(I_{k}\right) \rightarrow[0,1] .
$$

Thus, a continuous surjection from the disjoint union of $\pi^{-1}\left(I_{k}\right)$ 's to $[0,1]$ is defined and satisfies the desired property.

It remains to show that the domain of the $\eta_{k}$ 's can extend to the whole $Y$, satisfying the desired property. Let $J_{k}:=\left[t_{k}+\varepsilon, t_{k+1}-\varepsilon\right] \subset S^{1}$ be the interval between $I_{k}$ and $I_{k+1}$. Let us set $F_{k}=\pi^{-1}\left(t_{k}+\varepsilon\right)$ which is homeomorphic to $D^{2}$ or Mö. Let $G_{k}=\pi^{-1}\left(t_{k+1}-\varepsilon\right)$ which is homeomorphic to $F_{k}$.

Suppose that $F_{k} \approx D^{2}$. We recall that $D(-1) \subset \partial K_{1}\left(P^{2}\right)$ is defined as

$$
\left\{(x, y, z) \in \mathbb{R}^{3} \mid x^{2}+y^{2}-z^{2}=-1, x^{2}+y^{2} \leq 1\right\} /(x, y, z) \sim-(x, y, z) .
$$

We identify this as $D^{2}=\left\{(x, y) \mid x^{2}+y^{2} \leq 1\right\}$ by a map

$$
D(-1) \ni[x, y, z] \mapsto(x, y) \in D^{2} .
$$

Then, via $\varphi_{k}$, the map $\eta_{k}: F_{k} \rightarrow[0,1]$ can be identified as the map

$$
\theta^{\prime}: D^{2} \rightarrow[0,1] ;(x, y) \mapsto x^{2}+y^{2},
$$

namely, $\eta_{k}=\theta^{\prime} \circ \varphi_{k}^{-1}$. Similarly, $\eta_{k+1}=\theta^{\prime} \circ \varphi_{k+1}^{-1}$. Here, $\varphi_{k}$ and $\varphi_{k+1}$ are restricted on $D^{2} \subset \partial K_{1}\left(P^{2}\right)$. Let $r: D^{2} \rightarrow D^{2} ;(x, y) \mapsto(x,-y)$ be the reflection with respect to the $x$-axis. We note that $\theta^{\prime} \circ r=\theta^{\prime}$ and $r$ represents a unique non-trivial element 
of the mapping class group $\operatorname{MCG}\left(D^{2}\right)\left(\cong \mathbb{Z}_{2}\right)$ of $D^{2}$. By using Lemma [5.10, we obtain a homeomorphism

$$
\varphi_{k}^{\prime}: D^{2} \times J_{k} \rightarrow \pi^{-1}\left(J_{k}\right),
$$

respecting projections $\pi$ and $D^{2} \times J_{k} \rightarrow J_{k}$ such that $\varphi_{k}^{\prime}=\varphi_{k}$ on $F_{k}$ and either

$$
\begin{aligned}
& \varphi_{k}^{\prime}=\varphi_{k+1} \quad \text { on } G_{k}, \text { or } \\
& \varphi_{k}^{\prime}=\varphi_{k+1} \circ r \text { on } G_{k} .
\end{aligned}
$$

Hence,

$$
\eta_{k}^{\prime}=\theta^{\prime} \circ\left(\varphi_{k}^{\prime}\right)^{-1}: \pi^{-1}\left(J_{k}\right) \rightarrow[0,1]
$$

satisfies

$$
\eta_{k}^{\prime}=\eta_{k} \text { on } F_{k} \text { and } \eta_{k}^{\prime}=\eta_{k+1} \text { on } G_{k} .
$$

Therefore, if the fiber of $\pi$ on $J_{k}$ is a disk, then $\eta_{k}$ and $\eta_{k+1}$ extend to the map $\eta_{k}^{\prime}$ on $\pi^{-1}\left(J_{k}\right)$, satisfying the desired property.

Next, we assume that $F_{k} \approx$ Mö. We recall that $D(1)$ is

$$
\left\{(x, y, z)\left|x^{2}+y^{2}-x^{2}=1,\right| z \mid \leq 1\right\} /(x, y, z) \sim-(x, y, z) .
$$

Let us identify $D(1) \subset \partial K_{1}\left(P^{2}\right)$ as Mö defined by

$$
\text { Мö }=S^{1} \times[-1,1] /(x, s) \sim(-x,-s)
$$

via a map

$$
D(1) \ni[x, y, z] \mapsto\left[\frac{(x, y)}{\sqrt{x^{2}+y^{2}}}, z\right] \in \mathrm{Mö.}
$$

Then, $\eta_{k}$ is identified as a projection

$$
\theta^{\prime \prime}: \text { Mö } \ni[x, s] \mapsto s^{2} \in[0,1],
$$

via $\varphi_{k}$. Namely, $\eta_{k}=\theta^{\prime \prime} \circ \varphi_{k}^{-1}$ on $F_{k}$. We can see that $\eta_{k+1}=\theta^{\prime \prime} \circ \varphi_{k+1}^{-1}$ on $G_{k}$. Let us fix a homeomorphism $r:$ Mö $\rightarrow$ Mö defined by $r[x, s]=[\bar{x}, s]$, where $\bar{x}$ is the complex conjugate of $x$ in $S^{1} \subset \mathbb{C}$. Then, $r$ reverses the orientation of $\partial \mathrm{Mö}$. Hence, $r$ represents a unique non-trivial element of the mapping class group $\operatorname{MCG}(\mathrm{Mö}) \cong \mathbb{Z}_{2}$, and we note that $\theta^{\prime \prime} \circ r=\theta^{\prime \prime}$. By Lemma 5.10, there exists a homeomorphism

$$
\varphi_{k}^{\prime \prime}: \mathrm{Mö} \times J_{k} \rightarrow \pi^{-1}\left(J_{k}\right),
$$

respecting projections $\pi$ and Mö $\times J_{k} \rightarrow J_{k}$, such that $\varphi_{k}^{\prime \prime}=\varphi_{k}$ on $F_{k}$ and either

$$
\begin{aligned}
& \varphi_{k}^{\prime \prime}=\varphi_{k+1} \quad \text { on } G_{k}, \text { or } \\
& \varphi_{k}^{\prime \prime}=\varphi_{k+1} \circ r \text { on } G_{k} .
\end{aligned}
$$

Since $\theta^{\prime \prime}=\theta^{\prime \prime} \circ r$, we obtain a continuous surjection

$$
\eta_{k}^{\prime \prime}=\theta^{\prime \prime} \circ\left(\varphi_{k}^{\prime \prime}\right)^{-1}: \pi^{-1}\left(J_{k}\right) \rightarrow[0,1]
$$

satisfying

$$
\eta_{k}^{\prime \prime}=\eta_{k} \text { on } F_{k} \text { and } \eta_{k}^{\prime \prime}=\eta_{k+1} \text { on } G_{k} .
$$

By summarizing the above, we obtain a continuous surjection

$$
\eta: Y \rightarrow[0,1]
$$

satisfying the desired condition. 
Proof of Corollary 1.6. We may assume that $X$ has only one boundary component $\partial X$. By Theorem 1.5 there are decompositions

$$
M_{i}=M_{i}^{\prime} \cup M_{i}^{\prime \prime} \text { and } X=X^{\prime} \cup X^{\prime \prime}
$$

satisfying the following:

(1) $X^{\prime \prime}$ is a collar neighborhood of $\partial X$. We fix a homeomorphism $\varphi: \partial X \times$ $[0,1] \rightarrow X^{\prime \prime}$ such that $\varphi(\partial X \times\{0\})=\partial X$ and $\varphi(\partial X \times\{1\})=\partial X^{\prime}$.

(2) $M_{i}^{\prime}$ is a generalized Seifert fiber space over $X^{\prime} \approx X$. We fix a fibration $f_{i}^{\prime}: M_{i}^{\prime} \rightarrow X^{\prime}$ of it.

(3) $M_{i}^{\prime \prime}$ is a generalized solid torus or a generalized solid Klein bottle. We fix a projection $\pi_{i}: M_{i}^{\prime \prime} \rightarrow \partial X \approx S^{1}$ as (1.2) in Definition 1.4.

(4) The maps $f_{i}^{\prime}, \pi_{i}$ and $\varphi$ are compatible in the following sense. For any $x \in \partial X$

$$
\pi_{i}^{-1}(x) \cap \partial M_{i}^{\prime \prime}=\left(f_{i}^{\prime}\right)^{-1}(\varphi(x, 1))
$$

holds.

By Lemma 5.11, we obtain a continuous surjection

$$
\eta_{i}: M_{i}^{\prime \prime} \rightarrow[0,1]
$$

such that

(5) $\eta_{i}^{-1}(1)=\partial M_{i}^{\prime \prime}$

(6) Setting $g_{i}=\left(\pi_{i}, \eta_{i}\right): M_{i}^{\prime \prime} \rightarrow \partial X \times[0,1]$, the restriction of $g_{i}$ on

$$
g_{i}^{-1}(\partial X \times(0,1])
$$

is an $S^{1}$-bundle.

(7) For every $x \in \partial X, g_{i}^{-1}(x, 0)$ is one point set or a circle. The fiber of $g_{i}$ changes at $x \in \partial X$ if and only if the fiber of $\pi_{i}$ changes at $x$.

Then, the map

$$
f_{i}^{\prime \prime}=\varphi \circ g_{i}: M_{i}^{\prime \prime} \rightarrow X^{\prime \prime}
$$

satisfies

$$
f_{i}^{\prime}=f_{i}^{\prime \prime} \text { on } M_{i}^{\prime} \cap M_{i}^{\prime \prime} .
$$

Therefore, the gluing $f_{i}: M_{i} \rightarrow X$ of maps $f_{i}^{\prime}$ and $f_{i}^{\prime \prime}$ defined by

$$
f_{i}=\left\{\begin{array}{l}
f_{i}^{\prime} \text { on } M_{i}^{\prime} \\
f_{i}^{\prime \prime} \text { on } M_{i}^{\prime \prime}
\end{array}\right.
$$

is well-defined. The map $f_{i}$ satisfies the topological condition desired in Corollary 1.6.

From the proof of Theorem [1.5] and the construction of $X^{\prime \prime}$, for any $\varepsilon>0$ and large $i$, we can take $\pi_{i}: M_{i}^{\prime \prime} \rightarrow \partial X$ as an $\varepsilon$-approximation and $\varphi: \partial X \times[0,1] \rightarrow X^{\prime \prime}$ satisfying

$$
|| \varphi(x, t), \varphi\left(x^{\prime}, t^{\prime}\right)|-| x, x^{\prime}||<\varepsilon
$$

for any $x, x^{\prime} \in \partial X$ and $t, t^{\prime} \in[0,1]$. Then, one can show that $f_{i}$ is an approximation. 


\section{The case that $X$ is a Circle}

Let $\left\{M_{i}^{3}\right\}$ be a sequence of closed three-dimensional Alexandrov spaces with curvature $\geq-1$ and uniformly bounded diameter. Suppose that $M_{i}$ converges to a circle $X$. We will prove Theorem 1.7

Proof of Theorem 1.7. We first show

Lemma 6.1. For large $i, \Sigma_{x} \approx S^{2}$ for all $x \in M_{i}$. In particular, $M_{i}$ is a topological manifold.

Proof. Indeed, by Proposition 2.70, we may assume that diam $\Sigma_{x_{i}}$ is almost $\pi$ for each $x_{i} \in M_{i}$. It follows from Theorem 2.35 and $\partial M_{i}=\emptyset$ that $\Sigma_{x_{i}}$ is homeomorphic to the suspension over a circle, which is 2 -sphere. Therefore, by Theorem 2.34, $M_{i}$ is a topological manifold.

By taking a rescaling, we may assume that $M_{i}$ converges to the unit circle $X=S^{1}=\left\{e^{i \theta} \in \mathbb{C} \mid \theta \in[0,2 \pi]\right\}$. We take points $p^{+}:=1$ and $p^{-}:=-1 \in S^{1}$, and prepare points $p_{i}^{+}$and $p_{i}^{-} \in M_{i}$ converging to $p^{+}$and $p^{-}$, respectively. Let us set $q^{+}:=\sqrt{-1}$ and $q^{-}:=-\sqrt{-1} \in S^{1}$, and take $q_{i}^{+}, q_{i}^{-} \in M_{i}$ such that $q_{i}^{ \pm} \rightarrow q^{ \pm}$.

Let us take $\delta_{i}$ the diameter of a part of $\partial B\left(p_{i}, \pi / 2\right)$ which is GH-close to $q^{+} \in S^{1}$. We consider metric balls

$$
B_{i}^{+}:=B\left(p_{i}^{+}, \ell_{i}-\delta_{i}\right) \text { and } B_{i}^{-}:=B\left(p_{i}^{-}, \ell_{i}-\delta_{i}\right) .
$$

Here, $\ell_{i}=\left|p_{i}^{+}, p_{i}^{-}\right| / 2$. By the construction, $B_{i}^{+} \cap B_{i}^{-}=\emptyset$. We prove the next

Lemma 6.2. $B_{i}^{ \pm}$is homeomorphic to $F_{i}^{ \pm} \times[0,1]$. Here, $F_{i}^{ \pm}$is homeomorphic to $S^{2}, P^{2}, T^{2}$ or $K^{2}$.

Proof. We will prove this assertion only for $B_{i}^{+}$. Let us set $B_{i}:=B_{i}^{+}$and $p_{i}:=p_{i}^{+}$.

By Lemma 6.1, $M_{i}$ is a manifold. We will implicitly use this fact throughout the following argument.

Remark that

$$
\partial B_{i} \text { is disconnected. }
$$

If $B_{i}$ does not satisfy Assumption 2.28, then there exists a sequence $\hat{p}_{i} \in M_{i}$ where we may assume that $\hat{p}_{i}=p_{i}$ and $\partial B_{i} \approx \Sigma_{p_{i}} \approx S^{2}$. Hence $\partial B_{i}$ is connected. This is a contradiction.

Therefore, $B_{i}$ must satisfy Assumption 2.28, Then, by Theorem 2.27, there exist $\varepsilon_{i} \rightarrow 0$ and points $\hat{p}_{i} \in M_{i}$, where we may assume that $\hat{p}_{i}=p_{i}$, such that a limit $\left(Y, y_{0}\right):=\lim _{i \rightarrow \infty}\left(\frac{1}{\varepsilon_{i}} B_{i}, p_{i}\right)$ exists and has dimension $\geq 2$. We remark that $Y$ has a line, because $\tilde{L} q_{i}^{+} p_{i} q_{i}^{-} \rightarrow \pi$. It follows from Theorem 2.19 that $Y$ is isometric to $S \times \mathbb{R}$ for some non-negatively cured Alexandrov space $S$ of dimension at least one.

If $\operatorname{dim} S=2$, then by Theorem 2.34. $S$ has no boundary and the topology of $B_{i}$ can be determined. By the remark (6.1), $S$ is compact and, hence, $S$ is either homeomorphic to $S^{2}$ or $P^{2}$ or is isometric to a flat torus or a flat Klein bottle. Again, by using Theorem 2.34, we conclude that $B_{i} \approx S \times I$.

If $\operatorname{dim} S=1$, then by Theorems 1.3 and 1.5, the topology of $B_{i}$ can be determined. It follows from (6.1) that $S$ is compact. Hence $S$ is isometric to a circle or an interval. If $S$ is a circle, then $Y$ has no singular point. Then we can use Theorem 2.25, and therefore we conclude that $B_{i}$ is homeomorphic to $T^{2} \times I$ or 
$K^{2} \times I$. If $S$ is an interval, then by Theorem 1.5. $B_{i}$ is homeomorphic to $S^{2} \times I$, $P^{2} \times I$ or $K^{2} \times I$.

This completes the proof of Lemma 6.2.

Recall that $q_{i}^{ \pm}$are points in $M_{i}$ converging to $q^{ \pm}= \pm \sqrt{-1} \in S^{1}$. Let us consider

$$
D_{i}^{ \pm}:=B\left(q_{i}^{ \pm}, \pi / 2\right)-\operatorname{int}\left(B_{i}^{+} \cup B_{i}^{-}\right) .
$$

Let us set

By Lemma $\underline{6.2}, S_{i}^{ \pm} \approx F_{i}^{ \pm}$.

$$
S_{i}^{ \pm}:=B_{i}^{ \pm} \cap D_{i}^{+}
$$

Lemma 6.3. There is a homeomorphism $\phi_{i}: F_{i}^{+} \times[0,1] \rightarrow D_{i}^{+}$such that $\phi_{i}\left(F_{i}^{+} \times\{0\}\right)=S_{i}^{+}$and $\phi_{i}\left(F_{i}^{+} \times\{1\}\right)=S_{i}^{-}$.

Proof. Let $W_{i}$ be the component of $S\left(p_{i}, \ell_{i}\right)$ converging to $q=\sqrt{-1} \in S^{1}$. Recall that $\delta_{i}=\operatorname{diam} W_{i}$. Then $\delta_{i} \rightarrow 0$.

Let us take $q_{i} \in W_{i}$ and consider any limit $Y$ of a rescaling sequence:

$$
\left(\frac{1}{\delta_{i}} M_{i}, q_{i}\right) \rightarrow\left(Y, q_{\infty}\right)
$$

Let $\gamma_{\infty}^{ \pm}$be rays starting at $q_{\infty}$ which are limits of geodesics $q_{i} p_{i}^{ \pm}$. Since $\tilde{\angle} p_{i}^{+} q_{i} z_{i}^{-} \rightarrow$ $\pi, \gamma_{\infty}:=\gamma_{\infty}^{+} \cup \gamma_{\infty}^{-}$is a line in $Y$.

Let $W_{\infty}$ be the limit of $W_{i}$ under the convergence (6.2). By the choice of $\delta_{i}$, $\operatorname{diam} W_{\infty}=1$. We will prove that

Assertion 6.4. $Y$ is isometric to $W_{\infty} \times \mathbb{R}$. In particular, $\operatorname{dim} Y \geq 2$.

Proof of Assertion 6.4. Let us consider functions

$$
\begin{aligned}
f_{i}^{ \pm}(\cdot) & :=\tilde{d}_{i}\left(p_{i}^{ \pm}, \cdot\right)-\tilde{d}_{i}\left(p_{i}^{ \pm}, q_{i}\right), \\
b^{ \pm}(\cdot) & :=\lim _{t \rightarrow \infty} d\left(\gamma_{\infty}^{ \pm}(t), \cdot\right)-t .
\end{aligned}
$$

Here, $\tilde{d}_{i}$ is the original metric of $M_{i}$ multiplied by $1 / \delta_{i}$. The functions $b^{ \pm}$are the Busemann functions of the rays $\gamma_{\infty}^{ \pm}$. Then, we can show that $f_{i}^{ \pm}$converges to $b^{ \pm}$. Therefore, we obtain $W_{\infty}=\left(b^{+}\right)^{-1}(0)$. This completes the proof of Assertion 6.4 .

By Assertion 6.4, $\operatorname{dim} W_{\infty}=1$ or 2 . If $\operatorname{dim} W_{\infty}=2$, then by Theorem 2.34, we have a homeomorphism

with respect to functions $f_{i}^{ \pm}$and $b^{ \pm}$. Namely,

$$
\phi_{i}: D_{i}^{+} \approx W_{\infty} \times[-1,1]
$$

$$
\phi_{i}\left(\left(f_{i}^{ \pm}\right)^{-1}(t)\right)=\left(b^{ \pm}\right)^{-1}(t)
$$

whenever $t$ is near $\{-1,1\}$. In particular,

$$
S_{i}^{+}=\left(f_{i}^{+}\right)^{-1}(1) \approx\left(b^{ \pm}\right)^{-1}(0)=W_{\infty} \approx\left(f_{i}^{-}\right)^{-1}(1)=S_{i}^{-} .
$$

In this case, $W_{\infty} \approx S^{2}, P^{2}, T^{2}$ or $K^{2}$.

If $\operatorname{dim} W_{\infty}=1$, then $W_{\infty}$ is a circle or an interval. If $W_{\infty}$ is a circle, then by Theorem 2.25 and some flow argument, there is a circle fiber bundle

$$
\pi_{i}: D_{i}^{+} \rightarrow W_{\infty} \times[-1,1]
$$

such that $\pi_{i}^{-1}\left(W_{\infty} \times\{ \pm 1\}\right)=S_{i}^{ \pm}$. In this case, $S_{i}^{ \pm} \approx T^{2}$ or $K^{2}$. 
If $W_{\infty}$ is an interval, then by using Theorem 1.5 and some flow argument, we have a homeomorphism

$$
\phi_{i}: D_{i}^{+} \rightarrow S_{i}^{+} \times[-1,1]
$$

such that $\phi_{i}\left(S_{i}^{ \pm}\right)=S_{i}^{+} \times\{ \pm 1\}$. In this case, $S_{i}^{ \pm} \approx S^{2}, P^{2}$ or $K^{2}$.

This completes the proof of Lemma 6.3.

Let $F_{i}$ be a topological space homeomorphic to $F_{i}^{ \pm} \approx S_{i}^{ \pm}$. By Lemmas 6.2 and 6.3. we obtain homeomorphisms

$$
\begin{aligned}
& \varphi_{i}^{ \pm}: F_{i} \times[0,1] \rightarrow B_{i}^{ \pm}, \\
& \psi_{i}^{ \pm}: F_{i} \times[0,1] \rightarrow D_{i}^{ \pm}
\end{aligned}
$$

such that they send the boundaries to the boundaries. Therefore, $M_{i}=B_{i}^{+} \cup B_{i}^{-} \cup$ $D_{i}^{+} \cup D_{i}^{-}$is an $F_{i}$-bundle over $S^{1}$.

\section{The Case that $X$ is AN Interval}

Let $\left\{M_{i}\right\}$ be a sequence of three-dimensional closed Alexandrov spaces of curvature $\geq-1$ with $\operatorname{diam} M_{i} \leq D$. Suppose that $M_{i}$ converges to an interval $I$. Let $\partial I=\left\{p, p^{\prime}\right\}$, and let $p_{i}, p_{i}^{\prime} \in M_{i}$ converge to $p, p^{\prime}$, respectively. We divide $M_{i}$ into $M_{i}=B_{i} \cup D_{i} \cup B_{i}^{\prime}$, where $B_{i}=B\left(p_{i}, r\right), B_{i}^{\prime}=B\left(p_{i}^{\prime}, r\right)$ for small $r>0$, and $D_{i}:=M_{i}-\operatorname{int}\left(B_{i} \cup B_{i}^{\prime}\right)$.

Proof of Theorem 1.8. In a way similar to the proof of Lemma 6.3. we can prove that there exists a homeomorphism $\phi_{i}: F_{i} \times I \rightarrow D_{i}$ such that $\phi_{i}\left(F_{i} \times 0\right)=\partial B_{i}$ and $\phi_{i}\left(F_{i} \times 1\right)=\partial B_{i}^{\prime}$, where $F_{i}$ is homeomorphic to one of $S^{2}, P^{2}, T^{2}$ and $K^{2}$.

Next, we will find the topologies of $B_{i}$ (and $B_{i}^{\prime}$ ). If $B_{i}$ does not satisfy Assumption 2.28, then $B_{i}$ is homeomorphic to $D^{3}$ or $K_{1}\left(P^{2}\right)$. Hence, we may assume that there exist sequences $\delta_{i} \rightarrow 0$ and $\hat{p}_{i}$ such that a limit $\left(Y, y_{0}\right)=\lim _{i \rightarrow \infty} \frac{1}{\delta_{i}}\left(B_{i}, \hat{p}_{i}\right)$ exists, where we may assume that $\hat{p}_{i}=p_{i}$ and $Y$ is a non-compact non-negatively curved Alexandrov space of $\operatorname{dim} Y \geq 2$.

If $\operatorname{dim} Y=3$ with a soul $S \subset Y$, then Theorem 2.59 implies $B_{i}$ is homeomorphic to one of the following:

- $D^{3}, K_{1}\left(P^{2}\right)$ and $B(\mathrm{pt})$ if $\operatorname{dim} S=0$,

- $S^{1} \times D^{2}$ and $S^{1} \tilde{\times} D^{2}$ if $\operatorname{dim} S=1$, and

- $B(N(S))$ and $B\left(S_{2}\right)$ and $B\left(S_{4}\right)$ if $\operatorname{dim} S=2$.

Here, $N(S)$ is a non-trivial line bundle over a closed surface $S$ of non-negative curvature and $B(N(S))$ is a metric ball around $S$ in $N(S)$, and $B\left(S_{i}\right)$ is a metric ball around $S_{i}$ in $L_{i}=L\left(S_{i}\right)$ for $i=2,4$ (see 2.8.1). $B(N(S)$ ) is homeomorphic to one of the non-trivial twisted $I$-bundles over a closed surface $S$ with connected boundary. We determine the topology of $B(N(S))$ as follows: If $S \approx S^{2}, N(S)$ is isometric to $S \times \mathbb{R}$, which is a contradiction. If $S \approx P^{2}$, we have the line bundle $N(\hat{S})$ induced by the double covering $\pi: \hat{S} \rightarrow S$. Since $N(\hat{S})=\hat{S} \times \mathbb{R}$, we find that $N(S)=\hat{S} \times \mathbb{R} /(x, t) \sim(\sigma(x),-t)$, where $\sigma$ is the involution on $\hat{S}$ with $\hat{S} / \sigma=S$. Thus $B(N(S))$ is a twisted $I$-bundle over $P^{2}$, which is homeomorphic to $P^{3}$ - int $D^{3}$. If $S$ is homeomorphic to either $T^{2}$ or $K^{2}$, then $N(S)$ is a complete flat three-manifold. By $\mathrm{W}$, Theorem 3.5.1] we obtain that $B(N(S))$ is a twisted $I$-bundle over $T^{2}$, which is homeomorphic to Mö $\times S^{1}$, an orientable $I$-bundle $K^{2} \tilde{\times} I$ over $K^{2}$, and a non-trivial non-orientable $I$-bundle $K^{2} \hat{\times} I$ over $K^{2}$. 
If $\operatorname{dim} Y=2$ and $\partial Y=\emptyset$, then $Y$ is either homeomorphic to $\mathbb{R}^{2}$ or isometric to a flat cylinder or a flat Mobius strip.

Suppose that $Y \approx \mathbb{R}^{2}$. Let us denote by $m$ the number of essential singular points in $Y$. Then $m \leq 2$. When $m \leq 1$, Theorem 1.3 together with Lemma 4.5 implies that $B_{i} \approx S^{1} \times D^{2}$ or $B(\mathrm{pt})$. If $m=2$, then $Y$ is isometric to the envelope $\operatorname{dbl}\left(\mathbb{R}_{+} \times[0, \ell]\right)$ for some $\ell>0$. Let $B$ be a closed ball around $\{0\} \times[0, \ell]$ in $Y$. By Theorem 1.3. $B_{i}$ is a generalized Seifert fiber space over $B$ and its boundary $\partial B_{i}$ is homeomorphic to $T^{2}$ or $K^{2}$. We may assume that $B_{i}$ has actually two singular orbits over two singular points $(0,0)$ and $(0, \ell)$ in $Y$. Here, a singular orbit is either a $(2,1)$-type fiber corresponding to the core of $U_{2,1}$ or the interval fiber of $M_{\mathrm{pt}}$ in this case. The topology of $B_{i}$ is determined as follows: When two singular orbits are both (2,1)-type, int $B_{i}$ is homeomorphic to $U_{2,1}^{\prime} \cup_{\partial} U_{2,1}^{\prime}$. Since $U_{2,1}^{\prime}$ is an $\mathbb{R}$ bundle over Mö, int $B_{i}$ is an $\mathbb{R}$-bundle over $K^{2}$. By the boundary condition, $B_{i}$ is homeomorphic to $K^{2} \tilde{\times} I$ if $\partial B_{i} \approx T^{2}$ or $K^{2} \hat{\times} I$ if $\partial B_{i} \approx K^{2}$. When singular fibers of $B_{i}$ are $(2,1)$-type and an interval, int $B_{i}$ is homeomorphic to $U_{2,1}^{\prime} \cup_{\partial} M_{\mathrm{pt}}^{\prime}$. Then $B_{i}$ is homeomorphic to one of $B\left(S_{2}\right) \subset L_{2,1}$ with $S_{2} \approx P^{2}$. When $B_{i}$ has two singular interval fibers, $\operatorname{int} B_{i}$ is homeomorphic to $M_{\mathrm{pt}}^{\prime} \cup_{\partial} M_{\mathrm{pt}}^{\prime}$, which is $L_{4}$. Then $B_{i}$ is homeomorphic to $B\left(S_{4}\right)$.

If $Y$ is a flat cylinder, then $\partial B_{i}$ is not connected, and hence this case cannot happen.

If $Y$ is isometric to a flat Mobius strip, then $B_{i}$ is an $S^{1}$-bundle over Mö. Therefore, we have $B_{i} \approx \mathrm{Mö} \times S^{1}$ or $K^{2} \tilde{\times} I$.

If $\operatorname{dim} Y=2$ and $\partial Y \neq \emptyset$, then $Y$ is either isometric to a flat half cylinder $S^{1}(\ell) \times[0, \infty)$ or $[0, \ell] \times \mathbb{R}$ or homeomorphic to an upper half plane $\mathbb{R}_{+}^{2}=\mathbb{R} \times[0, \infty)$.

If $Y$ is a flat half cylinder, then $\partial Y$ has no essential singular point. Therefore, $B_{i}$ is a fiber bundle over $S^{1}$ with the fiber homeomorphic to $D^{2}$ or Mö. In other words, this is a generalized solid torus of type 0 or a generalized solid Klein bottle of type 0 .

If $Y \equiv[0, \ell] \times \mathbb{R}$, then $\partial B_{i}$ is not connected, and hence this case cannot happen.

Suppose that $Y$ is homeomorphic to $\mathbb{R}_{+}^{2}$. Let us set $m:=\sharp \operatorname{Ess}($ int $Y$ ) and $n:=\sharp \operatorname{Ess}(\partial Y)$. Then $m \leq 1$ and $n \leq 2$.

If $m=0$ and $n \leq 1$, then by Lemma [5.4, $B_{i}$ is homeomorphic to one of $D^{3}$, Mö $\times I$ or $K_{1}\left(P^{2}\right)$.

If $m=0$ and $n=2$, then $Y$ is isometric to $\mathbb{R}_{+} \times[0, \ell]$ for some $\ell>0$. Let $B:=[0, c] \times[0, \ell]$ for some $c>0$. By Corollary 1.6 , there is a continuous surjective map

$$
\pi: B_{i} \rightarrow B
$$

We may assume that $B_{i}$ has two topologically singular points converging to the corners $(0,0)$ and $(0, \ell)$ of $Y$. We divide $B$ into two domains,

$$
A_{j}=[0, c] \times\left\{y \in[0, \ell] \mid(-1)^{j}(y-\ell / 2) \geq 0\right\} \subset B,
$$

for $j=1,2$. Since $B_{i}$ has two topologically singular points, $\pi^{-1}\left(A_{j}\right) \approx K_{1}\left(P^{2}\right)$. Then, $B_{i}$ is homeomorphic to $K_{1}\left(P^{2}\right) \cup_{D^{2}} K_{1}\left(P^{2}\right)$ if $\pi^{-1}\left(A_{1} \cap A_{2}\right) \approx D^{2}$ or $K_{1}\left(P^{2}\right) \cup_{\text {Mö }} K_{1}\left(P^{2}\right)$ if $\pi^{-1}\left(A_{1} \cap A_{2}\right) \approx$ Mö. By Lemma 2.61 and Remark 2.62, $B_{i}$ is homeomorphic to $B(\mathrm{pt})$ or $B\left(S_{2}\right) \subset L_{2,2}$ with $S_{2} \approx S^{2}$.

If $m=1$, then $n=0$ and $Y$ is isometric to a cut envelope $\mathbb{R} \times[0, h] /(x, y) \sim$ $(-x, y)$ for some $h>0$. Let $B:=Y \cap\{(x, y) \mid x \leq r\}$ which is homeomorphic to $D^{2}$. By Theorem 1.5, there is a generalized Seifert fibration $\pi_{i}: W_{i} \rightarrow B$ such that 
$B_{i}$ is homeomorphic to a gluing of $W_{i}$ and $F_{i} \times[-r, r]$ via a homeomorphism

$$
\partial F_{i} \times[-r, r] \supset \partial F_{i} \times\{x\} \mapsto \pi_{i}^{-1}(x) \subset \pi_{i}^{-1}(\{(x, h) \in B \mid x \in[-r, r]\})
$$

for all $x \in[-r, r]$. Here, $F_{i}$ is $D^{2}$ or Mö. We may assume that $W_{i}$ contains a singular orbit over the singular point $(0,0) \in \operatorname{int} B$. If the singular orbit is a circle, then $W_{i}$ is isomorphic to a Seifert solid torus $V_{2,1}$ of $(2,1)$-type. Remark that $W_{i}$ can be regarded as an $I$-bundle over Mö, which corresponds to the preimage of the Seifert fibration over $\{0\} \times[0, h] \subset B$. Then, $B_{i}$ is isomorphic to an $I$-bundle over Mö $\cup_{\partial} F_{i}$. Therefore, it is $P^{2} \tilde{\times} I \approx P^{3}-\operatorname{int} D^{3}$ if $F_{i} \approx D^{2}$ or $K^{2} \hat{\times} I$ if $F_{i} \approx$ Mö. If the singular orbit is an interval, then Theorem 1.3 shows that $W_{i}$ is isomorphic to $M_{\mathrm{pt}}^{\prime}$. Recall that $B_{i}$ is homeomorphic to the union $W_{i} \cup F_{i} \times I$. Therefore, $B_{i}$ is homeomorphic to $B\left(S_{2}\right) \subset L_{2,2}$ with $S_{2} \approx S^{2}$ if $F_{i} \approx D^{2}$ or $B\left(S_{2}\right) \subset L_{2,3}$ with $S_{2} \approx P^{2}$ if $F_{i} \approx$ Mö.

This completes the proof of Theorem 1.8 .

\section{The CASE That $X$ IS A SINGLE-Point SET}

Lemma 8.1. If $M$ is a three-dimensional non-negatively curved closed Alexandrov space, then a finite covering of $M$ is $T^{3}, S^{1} \times S^{2}$ or simply-connected.

Proof. We may assume that $\left|\pi_{1}(M)\right|=\infty$. Then a universal covering $\tilde{M}$ of $M$ has a line. Thus, $\tilde{M}$ is isometric to the product $\mathbb{R}^{k} \times X_{0}$, where $1 \leq k \leq 3$ and $X_{0}$ is a $(3-k)$-dimensional non-negatively curved closed Alexandrov space.

- If $k=3$, then $\tilde{M}$ is the Euclidean space. Then a finite covering of $M$ is $T^{3}$.

- If $k=2$, then $X_{0}$ is a circle. Then $\tilde{M}$ is not simply-connected. This is a contradiction.

- If $k=1$, then $X_{0}$ is homeomorphic to $S^{2}$. Then a finite covering of $M$ is homeomorphic to $S^{1} \times S^{2}$.

Proof of Corollary 1.9. Let $\left\{M_{i}\right\}$ be a sequence of three-dimensional closed Alexandrov spaces of curvature $\geq-1$ with $\operatorname{diam} M_{i} \leq D$, which converges to a point $\{*\}$. Let $\delta_{i}:=\operatorname{diam} M_{i}$. Then the rescaled space $\frac{1}{\delta_{i}} M_{i}$ is an Alexandrov space with curvature $\geq-\delta_{i}^{2}$ having diameter one. Then, the limit $Y$ of the rescaled sequence $\frac{1}{\delta_{i}} M_{i}$ is a non-negatively curved Alexandrov space of $\operatorname{dimension} \geq 1$. If $\operatorname{dim} Y=1$, then $M_{i}$ is homeomorphic to a space in the conclusion of Theorems 1.7 and 1.8 . If $\operatorname{dim} Y=2$ and $\partial Y=\emptyset$, then $M_{i}$ is homeomorphic to a generalized Seifert fiber space having at most 4 singular fibers. If $\operatorname{dim} Y=2$ and $\partial Y \neq \emptyset$, then $M_{i}$ is homeomorphic to a space in the conclusion of Theorem 1.5 with at most 4 topologically singular points. If $\operatorname{dim} Y=3$, then by the Stability Theorem, $M_{i}$ is homeomorphic to $Y$. In this case, the topology of $Y$ is already obtained in Lemma 8.1 .

\section{ApPEndiX: $\varepsilon$-REgular COVERING OF THE BOUNDARY of an Alexandrov surface}

Let $X$ be an Alexandrov surface with non-empty compact boundary $\partial X$. Let us denote $C$ by a component of $\partial X$. The purpose of this section is to prove Lemma 9.9, which states the existence of an $\varepsilon$-regular covering of $C$, used in Section 5 .

We will first prepare a division of $C$ by consecutive arcs $\gamma_{1}, \gamma_{2}, \ldots, \gamma_{n}$ with $\partial \gamma_{\alpha}=$ $\left\{p_{\alpha}, p_{\alpha+1}\right\}$ and $p_{n+1}=p_{1}$. We next prove that this division makes the desired regular covering $\left\{B_{\alpha}, D_{\alpha}\right\}_{\alpha=1,2, \ldots, n}$ of $C$. 
For $\varepsilon>0$, we define

$$
S_{\varepsilon}(\partial X):=\left\{p \in \partial X \mid L\left(\Sigma_{p}\right) \leq \pi-\varepsilon\right\},
$$

where $L\left(\Sigma_{p}\right)$ is the length of $\Sigma_{p}$. Note that $S_{\varepsilon}(\partial X)$ is a finite set. We set

$$
R_{\varepsilon}(\partial X):=\partial X-S_{\varepsilon}(\partial X)
$$

and

$$
S_{\varepsilon}(C):=S_{\varepsilon}(\partial X) \cap C \text { and } R_{\varepsilon}(C):=R_{\varepsilon}(\partial X) \cap C .
$$

We review fundamental properties.

Lemma 9.1. For $\varepsilon>0$ and $p \in R_{\varepsilon}(\partial X)$, there exists $\delta>0$ such that for every $x \in B(p, \delta)-\partial X$, we have

$$
\left|\nabla d_{\partial X}\right|(x)>\cos \varepsilon \text {. }
$$

Proof. Suppose the contrary. Then, there are a sequence $\delta_{i} \rightarrow 0$ and $x_{i} \in B\left(p, \delta_{i}\right)-$ $\partial X$ such that $\left|\nabla d_{\partial X}\right|\left(x_{i}\right) \leq \cos \varepsilon$. Taking a subsequence, we consider the limit $x_{\infty} \in B\left(o_{p}, 1\right) \subset T_{p} X$ of $x_{i}$ under the convergence $\left(\frac{1}{\delta_{i}} X, p\right) \rightarrow\left(T_{p} X, o_{p}\right)$.

If $\left|\partial T_{p} X, x_{\infty}\right|>0$, then

$$
\left|\nabla d_{\partial T_{p} X}\right|\left(x_{\infty}\right)>-\cos \left(\pi-\frac{\varepsilon}{2}\right)=\cos \left(\frac{\varepsilon}{2}\right) .
$$

By the lower-semicontinuity of angles,

$$
\liminf _{i \rightarrow \infty}\left|\nabla d_{\partial X}\right|\left(x_{i}\right) \geq\left|\nabla d_{\partial T_{p} X}\right|\left(x_{\infty}\right) .
$$

This implies a contradiction.

When $\left|\partial T_{p} X, x_{\infty}\right|=0$, we take $y_{\infty} \in B\left(o_{p}, 1\right)-U\left(\partial T_{p} X, 1 / 2\right)$ such that

$$
\frac{\left|\partial T_{p} X, y_{\infty}\right|}{\left|x_{\infty}, y_{\infty}\right|}=\cos \angle x_{\infty} y_{\infty} \partial T_{p} X>\cos \left(\frac{\varepsilon}{2}\right) \text {. }
$$

We take a sequence $y_{i} \in B\left(p, \frac{3 \delta_{i}}{2}\right)-U\left(\partial X, \frac{\delta_{i}}{4}\right)$ such that $y_{i} \rightarrow y_{\infty}$ under the convergence $\left(\frac{1}{\delta_{i}} X, p\right) \rightarrow\left(T_{p} X, o_{p}\right)$. Since the distance function $d_{\partial X}$ is $\lambda$-concave for some $\lambda$ on $\operatorname{int} X$,

$$
\begin{aligned}
\frac{\left|\partial X, y_{i}\right|-\left|\partial X, x_{i}\right|}{\left|x_{i}, y_{i}\right|} & \leq \frac{\lambda}{2}\left|x_{i} y_{i}\right|+\left(d_{\partial X}\right)_{x_{i}}^{\prime}\left(\uparrow_{x_{i}}^{y_{i}}\right) \\
& \leq \frac{\lambda}{2}\left|x_{i} y_{i}\right|+\left|\nabla d_{\partial X}\right|\left(x_{i}\right) .
\end{aligned}
$$

Remark that $x_{i} y_{i} \subset \operatorname{int} X$ (Remark 9.2 , later). It is obvious that

$$
\frac{\left|\partial X, y_{i}\right|-\left|\partial X, x_{i}\right|}{\left|x_{i} y_{i}\right|} \rightarrow \frac{\left|\partial T_{p} X, y_{\infty}\right|}{\left|x_{\infty}, y_{\infty}\right|}(\text { as } i \rightarrow \infty) \text {. }
$$

Therefore, we conclude that

$$
\cos (\varepsilon / 2) \leq \cos \varepsilon
$$

This is a contradiction. Therefore, we have the conclusion of Lemma 9.1 .

Remark 9.2. The interior of an Alexandrov space is strictly convex. In fact, let $p, q \in \operatorname{int} M$. For every $x, y \in \operatorname{int}(p q)$ (the relative interior), $\Sigma_{x} \equiv \Sigma_{y}$ ([Pet Para $]$ ). If $x$ is near $p$, then $x \in \operatorname{int} M$, and hence $\partial \Sigma_{x}=\emptyset$. Then $\partial \Sigma_{y}=\emptyset$. Therefore, $p q \subset \operatorname{int} M$. 
Corollary 9.3. For any $\varepsilon, s>0$, there is $\delta_{1}>0$ such that

$$
\left|\nabla d_{\partial X}\right|>\cos \varepsilon
$$

on $B\left(\partial X, \delta_{1}\right)-\left(\partial X \cup U\left(S_{\varepsilon}(\partial X), s\right)\right)$.

Proof. The proof is provided by Lemma 9.1 and the Lebesgue covering lemma.

Lemma 9.4. For any $\varepsilon>0$, there is $\delta_{2}>0$ such that

$$
B\left(\partial X, \delta_{2}\right)-\partial X
$$

is $(2, \varepsilon)$-strained.

Proof. For any $p \in \partial X$, there is $\delta_{p}>0$ such that

$$
B\left(p, \delta_{p}\right)-\{p\}
$$

has no $\varepsilon^{\prime}$-critical point for $d_{p}$, where, $\varepsilon^{\prime} \ll \varepsilon$. Therefore, $B\left(p, \delta_{p}\right)-\partial X$ is $\left(1, \varepsilon^{\prime}\right)$ strained, and hence this is $(2, \varepsilon)$-strained. Since $\partial X$ is compact, there is $\delta>0$ such that, for any $p \in \partial X$, there exists $q \in \partial X$ with $B(p, \delta) \subset B\left(q, \delta_{q}\right)$. Therefore, $B(\partial X, \delta)-\partial X$ is $(2, \varepsilon)$-strained.

From now on, we use the notation $\tilde{\angle}(A ; B, C)$ defined as follows. Let $A, B$ and $C$ be positive numbers satisfying a part of the triangle inequality: $B+C \geq A$ and $A+C \geq B$. If $A+B \geq C$, then taking a geodesic triangle $\triangle a b c$ in the hyperbolic plane $\mathbb{H}^{2}$ with side lengths $|a b|=C,|b c|=A$ and $|c a|=B$, we set $\tilde{\angle}(A ; B, C):=\angle b a c$. Otherwise, $\tilde{\angle}(A ; B, C):=0$.

Let us start to construct a division of $C \subset \partial X$ to construct an $\varepsilon$-regular covering. Let us fix a small positive number $\varepsilon>0$.

Lemma 9.5. For any $p \in \partial X$, there is $s>0$ such that for any $q \in B(p, s) \cap$ $\partial X-\{p\}$ and $x \in \widehat{p q}-(\{q\} \cup U(p,|p q| / 2))$, we have

$$
\tilde{\angle}(|p q| ;|p x|, L(\widehat{x q}))>\pi-\varepsilon .
$$

Here, $\widehat{p q}$ is an arc joining $p$ and $q$ in $\partial X$. In particular,

$$
\tilde{\angle} p x q>\pi-\varepsilon \text {. }
$$

Proof. Suppose the contrary. Then, there are $p \in \partial X, s_{i} \rightarrow 0, q_{i} \in S\left(p, s_{i}\right) \cap \partial X$ and $x_{i} \in \widehat{p q}_{i}-\left(\left\{q_{i}\right\} \cup U\left(p,\left|p q_{i}\right| / 2\right)\right)$ such that

$$
\tilde{L}\left(\left|p q_{i}\right| ;\left|p x_{i}\right|, L\left(\widehat{x_{i} q_{i}}\right)\right) \leq \pi-\varepsilon .
$$

Taking a subsequence, we may assume that $q_{i}, x_{i}$ converges to $q_{\infty}, x_{\infty}$, respectively, under the convergence $\left(\frac{1}{s_{i}} X, p\right) \rightarrow\left(T_{p} X, o_{p}\right)$. Then, $q_{\infty} \in \partial T_{p} X,\left|o_{p}, q_{\infty}\right|=1$ and $x_{\infty} \in o_{p} q_{\infty}$.

If $x_{\infty} \neq q_{\infty}$, then

$$
\lim _{i \rightarrow \infty} \tilde{L}\left(\left|p q_{i}\right| ;\left|p x_{i}\right|, L\left(\widehat{x_{i} q_{i}}\right)\right)=\tilde{L} o_{p} x_{\infty} q_{\infty}=\pi .
$$

This is a contradiction.

Otherwise, $x_{\infty}=q_{\infty}$. We take $r_{\infty} \in \partial T_{p} X$ such that

$$
q_{\infty} \in o_{p} r_{\infty},\left|o_{p}, r_{\infty}\right|>3 / 2 \text {. }
$$

We choose $r_{i} \in \partial X$ such that $r_{i} \rightarrow r_{\infty}$ as $i \rightarrow \infty$ under the convergence $\left(\frac{1}{s_{i}} X, p\right) \rightarrow$ $\left(T_{p} X, o_{p}\right)$. Since $\widehat{x_{i} r_{i}}$ is a quasigeodesic containing $q_{i}$, by the comparison theorem for quasigeodesics $\mathrm{PP} \mathrm{QG}$, we have

$$
\tilde{L}\left(\left|p q_{i}\right| ;\left|p x_{i}\right|, L\left(\widehat{x_{i} q_{i}}\right)\right) \geq \tilde{L}\left(\left|p r_{i}\right| ;\left|p x_{i}\right|, L\left(\widehat{x_{i} r_{i}}\right)\right) .
$$


Since $L\left(\widehat{x_{i} r_{i}}\right) / s_{i} \rightarrow\left|x_{\infty} r_{\infty}\right|(\mid \widehat{P P Q G})$, we obtain

$$
\tilde{L}\left(\left|p r_{i}\right| ;\left|p x_{i}\right|, L\left(\widehat{x_{i} r_{i}}\right)\right) \rightarrow \tilde{L} o_{p} x_{\infty} r_{\infty}=\pi .
$$

This is a contradiction.

Lemma 9.6. For $p \in R_{\varepsilon}(\partial X)$, there is $s>0$ such that for any $q \in B(p, s) \cap$ $\partial X-\{p\}$ and $x \in \widehat{p q}-\{p, q\}$, we have

$$
\tilde{L}(|p q| ;|p x|, L(\widehat{x q}))>\pi-\varepsilon \text { or } \tilde{L}(|p q| ; L(\widehat{p x}),|x q|))>\pi-\varepsilon .
$$

In particular, $\tilde{\angle} p x q>\pi-\varepsilon$.

Proof. Suppose the contrary. Then there are $p \in \partial X, s_{i} \rightarrow 0, q_{i} \in S\left(p, s_{i}\right) \cap \partial X$ and $x_{i} \in \widehat{p q}_{i}-\left\{p, q_{i}\right\}$ such that

$$
\begin{aligned}
& \tilde{L}\left(\left|p q_{i}\right| ;\left|p x_{i}\right|, L\left(\widehat{x_{i} q_{i}}\right)\right) \leq \pi-\varepsilon \text { and } \\
& \left.\tilde{L}\left(\left|p q_{i}\right| ; L\left(\widehat{p x_{i}}\right),\left|x_{i} q_{i}\right|\right)\right) \leq \pi-\varepsilon .
\end{aligned}
$$

We may assume that $q_{i}$ and $x_{i}$ converge to $q_{\infty}$ and $x_{\infty}$, respectively, under the convergence $\left(\frac{1}{s_{i}} X, p\right) \rightarrow\left(T_{p} X, o_{p}\right)$. Then, $q_{\infty} \in \partial T_{p} X,\left|o_{p} q_{\infty}\right|=1$ and $x_{\infty} \in o_{p} q_{\infty}$.

If $q_{\infty} \neq o_{p}$, then by the same argument as in the proof of Lemma 9.5, we have

$$
\tilde{L}\left(\left|p q_{i}\right| ;\left|p x_{i}\right|, L\left(\widehat{x_{i} q_{i}}\right)\right) \rightarrow \pi \text {. }
$$

This is a contradiction to (9.1).

Otherwise, $q_{\infty}=o_{p}$. We take $r_{\infty} \in \partial T_{p} X \cap S\left(o_{p}, 1\right)-\left\{q_{\infty}\right\}$ and $r_{i} \in \partial X$ such that $r_{i} \rightarrow r_{\infty}$. Since $\widehat{x_{i} r_{i}}$ is a quasigeodesic containing $p$, by the comparison theorem for quasigeodesics, we have

$$
\left.\left.\tilde{L}\left(\left|p q_{i}\right| ; L\left(\widehat{p x_{i}}\right),\left|x_{i} q_{i}\right|\right)\right) \geq \tilde{L}\left(\left|r_{i} q_{i}\right| ; L\left(\widehat{r_{i} x_{i}}\right),\left|x_{i} q_{i}\right|\right)\right) .
$$

Since $L\left(\widehat{r_{i} x_{i}}\right) / s_{i} \rightarrow\left|r_{\infty} o_{p}\right|$, we obtain

$$
\left.\tilde{L}\left(\left|r_{i} q_{i}\right| ; L\left(\widehat{r_{i} x_{i}}\right),\left|x_{i} q_{i}\right|\right)\right) \rightarrow \tilde{L} q_{\infty} o_{p} r_{\infty}>\pi-\varepsilon .
$$

This is a contradiction to (9.2).

Definition 9.7. Let $\gamma=\widehat{p q}$ be an arc joining $p$ and $q$ in $\partial X$. We say that $\gamma$ is strictly $\varepsilon$-strained by $\partial \gamma=\{p, q\}$ if

$$
\tilde{\angle} p x q>\pi-\varepsilon \text { for all } x \in \operatorname{int} \gamma,
$$

and if setting $\xi$ and $\eta$ as the directions of quasigeodesics $\widehat{x p}$ and $\widehat{x q}$ at $x$, respectively, we have

$$
\angle\left(\xi, \uparrow_{x}^{p}\right)<\varepsilon \text { and } \angle\left(\eta, \uparrow_{x}^{q}\right)<\varepsilon .
$$

Remark that an arc $\widehat{p q}$ in Lemma 9.6 is strictly $\varepsilon$-strained by $\{p, q\}$. Indeed, we assume that $\tilde{L}(|p q| ;|p x|, L(\widehat{x q}))>\pi-\varepsilon$ for some $x \in \operatorname{int} \widehat{p q}$. We obtain $\tilde{\angle} p x q \geq$ $\tilde{\angle}(|p q| ;|p x|, L(\widehat{x q}))>\pi-\varepsilon$. Let $\xi$ and $\eta$ be the directions of $\widehat{x p}$ and $\widehat{x q}$ at $x$, respectively. Since $\operatorname{dim} X=2, \xi$ and $\eta$ attain the diameter of $\Sigma_{x}$, i.e.

$$
\angle(\xi, \eta)=L\left(\Sigma_{x}\right)
$$

Hence, we have

$$
\begin{aligned}
\angle(\xi, \eta) & =\angle\left(\xi, \uparrow_{x}^{p}\right)+\angle\left(\uparrow_{x}^{p}, \eta\right) \\
& =\angle\left(\xi, \uparrow_{x}^{p}\right)+\angle\left(\uparrow_{x}^{p}, \uparrow_{x}^{q}\right)+\angle\left(\uparrow_{x}^{q}, \eta\right) \\
& \geq \angle\left(\uparrow_{x}^{p}, \uparrow_{x}^{q}\right) \geq \tilde{\angle} p x q>\pi-\varepsilon .
\end{aligned}
$$


Since $L\left(\Sigma_{p}\right) \leq \pi$, we obtain

$$
\angle\left(\xi, \uparrow_{x}^{p}\right)+\angle\left(\uparrow_{x}^{q}, \eta\right)<\varepsilon .
$$

In particular, (9.4) holds.

Let us fix a component $C$ of $\partial X$. By Lemma 9.5 and $\sharp S_{\varepsilon}(C)<\infty$, there is $s>0$ such that for every $p \in S_{\varepsilon}(C)$, taking $q^{+}, q^{-} \in S(p, s) \cap C$, we have

$$
\tilde{\angle}\left(\left|p q^{ \pm}\right| ;|p x|, L\left(\widehat{x q^{ \pm}}\right)\right)>\pi-\varepsilon
$$

for all $x \in \beta_{p}^{ \pm}-\left(U(p, s / 2) \cup\left\{q^{ \pm}\right\}\right)$, where $\beta_{p}^{ \pm}:=\widehat{p q^{ \pm}}$.

Let us consider the set

$$
C-U\left(S_{\varepsilon}(C), s\right)=C-\bigcup_{p \in S_{\varepsilon}(C)} \operatorname{int}\left(\beta_{p}^{+} \cup \beta_{p}^{-}\right) .
$$

This consists of finitely many arcs. We prove that each component $K$ of it is divided into finitely many strictly $\varepsilon$-strained arcs.

Lemma 9.8. Let $K$ be an arc in $R_{\varepsilon}(C)$ with $\partial K=\{p, q\}$. There are consecutive arcs $\gamma_{\alpha}=\widehat{p_{\alpha} p_{\alpha+1}}, \alpha=1,2, \ldots, n$ with $p_{1}=p$ and $p_{n+1}=q$ such that each $\gamma_{\alpha}$ is strictly $\varepsilon$-strained by $\left\{p_{\alpha}, p_{\alpha+1}\right\}$.

Proof. By repeatedly using Lemma 9.6, we have a set $\Phi$ of consecutive arcs starting from $p$ contained in $K$,

$$
\Phi=\left\{\gamma_{1}, \gamma_{2}, \ldots, \gamma_{n}\right\}
$$

such that each $\gamma_{\alpha}$ is strictly $\varepsilon$-strained by $\partial \gamma_{\alpha}$. Here, "consecutive arcs starting from $p$ " means that each $\gamma_{\alpha}$ forms $\gamma_{\alpha}=\widehat{p_{\alpha} p_{\alpha+1}} \subset K$ and $p_{1}=p$.

In what follows, $\Phi$ denotes any such finite sequence of arcs as above. Let us set

$$
L(\Phi):=\sum_{\alpha=1}^{n} L\left(\gamma_{\alpha}\right)
$$

We consider the value $\ell:=\sup _{\Phi} L(\Phi)$. Since $\gamma_{\alpha}$ are consecutive and contained in $K$, we have $\ell \leq L(K)$. To prove the lemma, we show that there exists $\Phi$ with $L(\Phi)=L(K)$.

If $\ell=L(K)$, then there is $\Phi=\left\{\gamma_{\alpha}\right\}_{1 \leq \alpha \leq n}$ such that $p_{n}$ is arbitrarily close to $q$. If there is $\Phi$ with $L(\Phi)=L(K)$, then the proof is done. Otherwise, by using Lemma 9.6 for $q$, we can take $\Phi$ such that $\gamma_{n+1}:=\widehat{p_{n} q}$ is strictly $\varepsilon$-strained by $\partial \gamma_{n+1}$. Then we obtain an extension

$$
\tilde{\Phi}:=\Phi \cup\left\{\gamma_{n+1}\right\}
$$

of $\Phi$ with $L(\tilde{\Phi})=L(K)$. This is a contradiction. Therefore, if $\ell=L(K)$, then there is $\Phi$ attaining $L(\Phi)=L(K)$.

We assume that $\ell<L(K)$. By a similar argument as above, we have $\Phi=$ $\left\{\gamma_{\alpha}\right\}_{1 \leq \alpha \leq n}$ such that $L(\Phi)=\ell$. Again, by a similar argument as above, we have an extension $\tilde{\Phi}$ of $\Phi$. Hence $L(\tilde{\Phi})>\ell$. This is a contradiction.

By Lemma 9.8 and the decomposition (9.5), we obtain a division of $C$ :

$$
C=\left(\bigcup_{p \in S_{\varepsilon}(C)} \beta_{p}^{+} \cup \beta_{p}^{-}\right) \cup\left(\bigcup_{K} \bigcup_{i=1}^{n_{K}} \gamma_{\alpha}^{K}\right),
$$


where $\beta_{p}^{ \pm}:=\widehat{p q^{ \pm}}$and $K$ denotes any arc component of $C-U\left(S_{\varepsilon}(C), s\right)$. For each $K, \gamma_{\alpha}^{K}\left(1 \leq \alpha \leq n_{K}\right)$ expresses a strictly $\varepsilon$-strained arc by $\partial \gamma_{\alpha}^{K}$, obtained in Lemma 9.8 . $C$.

By using a division (9.6) of $C$, we prove the existence of an $\varepsilon$-regular covering of

Lemma 9.9. There is an $\varepsilon$-regular covering of $C$.

Proof. Let us fix a division of $C$ obtained as (9.6). Fixing a component $K$, we write $n=n_{K}, \gamma_{\alpha}=\gamma_{\alpha}^{K}$. Each $\gamma_{\alpha}$ forms $\gamma_{\alpha}=\widehat{p_{\alpha} p_{\alpha+1}}$. We take a small positive number $r$ such that

$$
\begin{aligned}
& \left|\nabla d_{p_{\alpha}}\right|>1-\varepsilon \text { on } B\left(p_{\alpha}, 2 r\right)-\left\{p_{\alpha}\right\} \text { for all } \alpha, \\
& B_{\alpha} \cap B_{\alpha^{\prime}}=\emptyset \text { for all } \alpha \neq \alpha^{\prime},
\end{aligned}
$$

where $B_{\alpha}:=B\left(p_{\alpha}, r\right)$.

By the condition (9.3), there is a small positive number $\delta$ with $\delta \ll r$ such that, setting

$$
D_{\alpha}:=B\left(\gamma_{\alpha}, \delta\right)-\operatorname{int}\left(B_{\alpha} \cup B_{\alpha+1}\right),
$$

we have

$$
\tilde{\angle} p_{\alpha} x p_{\alpha+1}>\pi-\varepsilon
$$

for all $x \in D_{\alpha}$. Further, by (9.3) and (9.4), $\delta$ can be chosen that for every $x \in D_{\alpha}$ and $y \in C$ with $|x C|=|x y|$, we have

$$
\left|\angle p_{\alpha} x y-\pi / 2\right|<2 \varepsilon \text { and }\left|\angle p_{\alpha+1} x y-\pi / 2\right|<2 \varepsilon \text {. }
$$

To use later, we set

$$
\Phi_{K}:=\left\{B_{\alpha}\right\}_{1 \leq \alpha \leq n} \cup\left\{D_{\alpha}\right\}_{1 \leq \alpha \leq n-1} .
$$

For $p \in S_{\varepsilon}(C)$, there are unique components $K^{+}$and $K^{-}$of $C-U\left(S_{\varepsilon}(C, s)\right)$ with $\beta_{p}^{ \pm} \cap K^{ \pm} \neq \emptyset$. We take unique elements $q^{ \pm} \in \beta_{p}^{ \pm} \cap K^{ \pm}$. Recall that $s>0$ is a small positive number satisfying the conclusion of Lemma 9.5 for $p$, and $\left|\nabla d_{p}\right|>1-\varepsilon$ on $B(p, s)-\{p\}$. For $q^{ \pm} \in K^{ \pm}$, we provided numbers $r^{ \pm}$satisfying (9.7) and (9.8), above. Let us set

$$
B_{p}:=B(p, s / 2) \text { and } D_{p}^{ \pm}:=B\left(\beta_{p}^{ \pm}, \delta\right)-\operatorname{int}\left(B_{p} \cup B\left(q^{ \pm}, r^{ \pm}\right)\right) .
$$

If we retake $\delta$ small enough, we have

$$
\left|\angle p x q^{ \pm}-\pi / 2\right|<\varepsilon
$$

for all $x \in D_{p}^{ \pm}$.

Thus, we obtain an $\varepsilon$-regular covering

$$
\left\{B_{p}, D_{p}^{ \pm}\right\}_{p \in S_{\varepsilon}(C)} \cup \bigcup_{K} \Phi_{K}
$$

of $C$. 


\section{REFERENCES}

[BBI] Dmitri Burago, Yuri Burago, and Sergei Ivanov, A course in metric geometry, Graduate Studies in Mathematics, vol. 33, American Mathematical Society, Providence, RI, 2001. MR.1835418 (2002e:53053)

[BGP] Yu. Burago, M. Gromov, and G. Perel'man, A. D. Aleksandrov spaces with curvatures bounded below (Russian, with Russian summary), Uspekhi Mat. Nauk 47 (1992), no. 2(284), 3-51, 222, DOI 10.1070/RM1992v047n02ABEH000877; English transl., Russian Math. Surveys 47 (1992), no. 2, 1-58. MR.1185284(93m:53035)

[BH] Martin R. Bridson and André Haefliger, Metric spaces of non-positive curvature, Grundlehren der Mathematischen Wissenschaften [Fundamental Principles of Mathematical Sciences], vol. 319, Springer-Verlag, Berlin, 1999. MR.1744486 (2000k:53038)

[CaGe] Jianguo Cao and Jian Ge, A simple proof of Perelman's collapsing theorem for 3manifolds, J. Geom. Anal. 21 (2011), no. 4, 807-869, DOI 10.1007/s12220-010-9169-5. MR2836584

[ChGr] Jeff Cheeger and Detlef Gromoll, On the structure of complete manifolds of nonnegative curvature, Ann. of Math. (2) 96 (1972), 413-443. MR0309010 (46 \#8121)

[E] D. B. A. Epstein, Curves on 2-manifolds and isotopies, Acta Math. 115 (1966), 83107. MR0214087 (35 \#4938)

[FY] Kenji Fukaya and Takao Yamaguchi, The fundamental groups of almost non-negatively curved manifolds, Ann. of Math. (2) 136 (1992), no. 2, 253-333, DOI 10.2307/2946606. MR1185120 (93h:53041)

[GP] Karsten Grove and Peter Petersen, A radius sphere theorem, Invent. Math. 112 (1993), no. 3, 577-583, DOI 10.1007/BF01232447. MR1218324 (94e:53034)

[Kap Rest] Vitali Kapovitch, Restrictions on collapsing with a lower sectional curvature bound, Math. Z. 249 (2005), no. 3, 519-539, DOI 10.1007/s00209-004-0715-3. MR.2121738 (2005k:53048)

[Kap Stab] Vitali Kapovitch, Perelman's stability theorem, Surveys in differential geometry. Vol. XI, Surv. Differ. Geom., vol. 11, Int. Press, Somerville, MA, 2007, pp. 103-136, DOI 10.4310/SDG.2006.v11.n1.a5. MR2408265 (2009g:53057)

[KL] B. Kleiner and J. Lott, Locally collapsed 3-manifolds, preprint.

[KL orb] B. Kleiner and J. Lott, Geometrization of three-dimensional orbifolds via Ricci flow, preprint.

[KMS] Kazuhiro Kuwae, Yoshiroh Machigashira, and Takashi Shioya, Sobolev spaces, Laplacian, and heat kernel on Alexandrov spaces, Math. Z. 238 (2001), no. 2, 269-316, DOI 10.1007/s002090100252. MR.1865418 (2002m:58052)

[L] A. Lytchak, Differentiation in metric spaces, Algebra i Analiz 16 (2004), no. 6, 128161, DOI 10.1090/S1061-0022-05-00888-5; English transl., St. Petersburg Math. J. 16 (2005), no. 6, 1017-1041. MR2117451 (2005j:53039)

[Milka] A. D. Milka, Metric structure of a certain class of spaces that contain straight lines (Russian), Ukrain. Geometr. Sb. Vyp. 4 (1967), 43-48. MR0256327 (41 \#983)

[M] Ayato Mitsuishi, A splitting theorem for infinite dimensional Alexandrov spaces with nonnegative curvature and its applications, Geom. Dedicata 144 (2010), 101-114, DOI 10.1007/s10711-009-9390-1. MR2580420(2011a:53062)

[MT] J. Morgan and G. Tian, Completion of the proof of the geometrization conjecture, preprint.

[N] M. A. Natsheh, On the involutions of closed surfaces, Arch. Math. (Basel) 46 (1986), no. 2, 179-183, DOI 10.1007/BF01197497. MR834833(88a:57072)

[O] Yukio Otsu, On manifolds of small excess, Amer. J. Math. 115 (1993), no. 6, 12291280, DOI 10.2307/2374965. MR.1254733(95i:53046)

[OS] Yukio Otsu and Takashi Shioya, The Riemannian structure of Alexandrov spaces, J. Differential Geom. 39 (1994), no. 3, 629-658. MR.1274133 (95e:53062)

[Per II] G. Perelman, A. D. Alexandrov's spaces with curvatures bounded from below. II, preprint.

[Per Elem] G. Perelman, Elements of Morse theory on Aleksandrov spaces (Russian, with Russian summary), Algebra i Analiz 5 (1993), no. 1, 232-241; English transl., St. Petersburg Math. J. 5 (1994), no. 1, 205-213. MR1220498 (94h:53054)

[Per DC] G. Perelman, DC Structure on Alexandrov Space, preprint. 
[Per Ent] G. Perelman, The entropy formula for the Ricci flow and its geometric applications, arXiv:math.DG / 0211159.

[Per Surg] G. Perelman, Ricci flow with surgery on three-manifolds, arXiv:math.DG / 0303109.

[PP QG] G. Perelman and A. Petrunin, Quasigeodesics and gradient curves in Alexandrov spaces, preprint.

[Pet QG] Anton Petrunin, Quasigeodesics in multidimensional Alexandrov spaces, ProQuest LLC, Ann Arbor, MI. Thesis (Ph.D.)-University of Illinois at Urbana-Champaign, 1995. MR2693118

[Pet Appl] Anton Petrunin, Applications of quasigeodesics and gradient curves, Comparison geometry (Berkeley, CA, 1993), Math. Sci. Res. Inst. Publ., vol. 30, Cambridge Univ. Press, Cambridge, 1997, pp. 203-219. MR1452875 (98m:53061)

[Pet Para] Anton Petrunin, Parallel transportation for Alexandrov space with curvature bounded below, Geom. Funct. Anal. 8 (1998), no. 1, 123-148, DOI 10.1007/s000390050050. MR 1601854 (98j:53048)

[Pet Semi] Anton Petrunin, Semiconcave functions in Alexandrov's geometry, Surveys in differential geometry. Vol. XI, Surv. Differ. Geom., vol. 11, Int. Press, Somerville, MA, 2007, pp. 137-201, DOI 10.4310/SDG.2006.v11.n1.a6. MR2408266 (2010a:53052)

[Pl] Conrad Plaut, Spaces of Wald curvature bounded below, J. Geom. Anal. 6 (1996), no. 1, 113-134, DOI 10.1007/BF02921569. MR1402389 (97j:53043)

[SY00] Takashi Shioya and Takao Yamaguchi, Collapsing three-manifolds under a lower curvature bound, J. Differential Geom. 56 (2000), no. 1, 1-66. MR.1863020(2002k:53074)

[SY05] Takashi Shioya and Takao Yamaguchi, Volume collapsed three-manifolds with a lower curvature bound, Math. Ann. 333 (2005), no. 1, 131-155, DOI 10.1007/s00208-0050667-x. MR2169831 (2006j:53050)

[Sie] L. C. Siebenmann, Deformation of homeomorphisms on stratified sets. I, II, Comment. Math. Helv. 47 (1972), 123-136; ibid. 47 (1972), 137-163. MR0319207 (47 \#7752)

[W] Joseph A. Wolf, Spaces of constant curvature, 5th ed., Publish or Perish Inc., Houston, TX, 1984. MR928600 (88k:53002)

[Y91] Takao Yamaguchi, Collapsing and pinching under a lower curvature bound, Ann. of Math. (2) 133 (1991), no. 2, 317-357, DOI 10.2307/2944340. MR:1097241|(92b:53067)

[Y conv] Takao Yamaguchi, A convergence theorem in the geometry of Alexandrov spaces (English, with English and French summaries), Actes de la Table Ronde de Géométrie Différentielle (Luminy, 1992), Sémin. Congr., vol. 1, Soc. Math. France, Paris, 1996, pp. 601-642. MR1427772 (97m:53078)

[Y 4-dim] Takao Yamaguchi, Collapsing 4-manifolds under a lower curvature bound, arXiv:1205.0323v1 [math.DG]

[Y ess] Takao Yamaguchi, Collapsing and essential covering, arXiv:1205.0441v1 [math.DG]

Mathematical Institute, Tohoku University, Sendai 980-8578, Japan

E-mail address: mitsuishi@math.tohoku.ac.jp

Institute of Mathematics, University of Tsukuba, Tsukuba 305-8571, Japan

E-mail address: takao@math.tsukuba.ac.jp

Current address: Department of Mathematics, Kyoto University, Kyoto 606-8502, Japan

E-mail address: takao@math.kyoto-u.ac.jp 